\title{
Relative Elliptic Theory
}

\author{
Vladimir Nazaikinskii* \\ Institute for Problems in Mechanics, Russian Academy of Sciences \\ e-mail: nazaik@math.uni-potsdam.de \\ Boris Sternin* \\ Independent University of Moscow \\ e-mail: sternine@mtu-net.ru
}

\begin{abstract}
This paper is a survey of relative elliptic theory (i.e. elliptic theory in the category of smooth embeddings), closely related to the Sobolev problem, first studied by Sternin in the 1960s. We consider both analytic aspects of the theory (the structure of the algebra of morphisms, ellipticity, Fredholm property) and topological aspects (index formulas and Riemann-Roch theorems). We also study the algebra of Green operators arising as a subalgebra of the algebra of morphisms.
\end{abstract}

Keywords: Sobolev problem, elliptic morphism, (co)boundary operator, Green operator, index, Riemann-Roch theorem.

2000 AMS classification: Primary: 58J05. Secondary: 58J20, 58J32, 58J40

\footnotetext{
*Supported by the DFG via a project with the Arbeitsgruppe "Partielle Differentialgleichungen und Komplexe Analysis," Institut für Mathematik, Universität Potsdam, and by RFBR grants Nos. 02-0100118, 02-01-06515 and 00-01-00161.
} 


\section{Contents}

Introduction $\quad 3$

Analytic Aspects of Relative Elliptic Theory 11

1 Classical objects. Lagrangian formalism $\quad 13$

1.1 Lagrangian manifolds with measure . . . . . . . . . . . . . . 13

1.2 Composition and transposition formulas . . . . . . . . . . 18

1.3 Symbols . . . . . . . . . . . . . . . . . . . 20

1.4 The product of symbols (twisted multiplication) . . . . . . . . . 26

2 Quantization and the algebra of operator morphisms 28

2.1 The main operators . . . . . . . . . . . . . . 28

2.2 Composition formulas and adjoint operators . . . . . . . . . . . . . 32

2.3 Algebras of operator morphisms . . . . . . . . . . . . . . 33

3 Elliptic morphisms and the index formula 36

3.1 The Green equation . . . . . . . . . . . . . . . . 36

3.2 Ellipticity and the Fredholm property . . . . . . . . . . . . . 40

3.3 The index theorem for elliptic morphisms . . . . . . . . . . . 45

4 The Sobolev problem $\quad 45$

4.1 Sobolev problems and elliptic morphisms . . . . . . . . . . . . . . . . . 45

4.2 The Fredholm property . . . . . . . . . . . . . . . 48

4.3 The Sobolev problem and the adjoint problem . . . . . . . . . . . 49

4.4 The index formula . . . . . . . . . . . . . . . . . 49

Topological Aspects of Relative Elliptic Theory 50

$\begin{array}{lll}5 & \text { Preliminaries } & 50\end{array}$

6 The metric trace of an elliptic operator $\quad 51$

7 The pullback of an elliptic operator $\quad 53$

7.1 The pullback of a pseudodifferential operator . . . . . . . . . . . 53

7.2 The regularized pullback . . . . . . . . . . . . . . . 54

7.3 The Riemann-Roch theorem for the regularized pullback . . . . . . . 56 
8 A finitely regularized pullback $\quad 56$

8.1 A counterexample . . . . . . . . . . . . . . 56

8.2 The pullback for linear principal symbols . . . . . . . . . . . . . 59

8.3 Pullbacks and exterior tensor products . . . . . . . . . . . 60

9 The pullback of geometric operators $\quad 62$

9.1 Group operators . . . . . . . . . . . . . . . . . 62

9.2 The Euler and Hirzebruch operators . . . . . . . . . . . . . 63

9.3 The Todd and Dirac operators . . . . . . . . . . . . . . . . . 67

Appendix. Fourier Integral Operators $\quad 67$

A.1 Homogeneous Lagrangian manifolds . . . . . . . . . . . . . . . 68

A.2 Local description of homogeneous Lagrangian manifolds . . . . . . . . . . . 69

A.3 Composition of homogeneous Lagrangian manifolds . . . . . . . . . . . 69

A.4 Definition of Fourier integral operators . . . . . . . . . . . . 70

A.5 Pseudodifferential operators as Fourier integral operators . . . . . . . . . 71

A.6 Boundedness theorems . . . . . . . . . . . . . . . . . 71

A.7 Composition theorems . . . . . . . . . . . . . . . 71

A.8 $L^{2}$-adjoints of Fourier integral operators $\ldots \ldots \ldots \ldots . \ldots . \ldots 72$

A.9 Fourier integral operators on sections of vector bundles . . . . . . . . 72

$\begin{array}{ll}\text { Bibliography } & 72\end{array}$

\section{Introduction}

1. By relative elliptic theory we mean elliptic theory associated with a pair $(M, X)$, where $M$ is a smooth closed manifold and $X$ a submanifold. Thus, relative elliptic theory is elliptic theory in the category of smooth embeddings.

A trivial (and noninteresting) example of a relative elliptic operator is given by a pair of elliptic operators on the manifolds $M$ and $X$, respectively. Such an operator can be represented in the form of a diagonal matrix operator

$$
\left(\begin{array}{cc}
D_{M} & 0 \\
0 & D_{X}
\end{array}\right): \begin{aligned}
& H^{s_{1}}(M) \\
& H^{s_{2}}(M)
\end{aligned} \longrightarrow \underset{H^{\sigma_{2}}(M)}{\oplus}
$$

with elliptic (pseudodifferential) operators $D_{M}$ and $D_{X}$ acting, say, in Sobolev spaces on $M$ and $X$. 
This trivial example, however, suggests that in the general case a relative elliptic operator should apparently be represented by a matrix of the form

$$
\left(\begin{array}{ll}
D_{M M} & D_{M X} \\
D_{X M} & D_{X X}
\end{array}\right)
$$

(where the subscripts show, from the right to the left, the direction of action of the corresponding operators), which is not necessarily diagonal. First, we note that a posteriori this guess proves to be true. However, it deserves a serious explanation. Indeed, the operators $D_{M X}$ and $D_{X M}$, representing the off-diagonal entries of this matrix, act from a function space on one manifold to a function space on another manifold and hence, unlike $D_{M M}$ and $D_{X X}$, are by no means pseudodifferential operators, at least if the term is understood in the classical sense. Thus, what is the nature of these operators?

Let us try to find some candidates for these operators. First, consider the operator $D_{X M}$. This operator acts from a function space on $M$ into a function space on the submanifold $X$. The most natural operator of this kind is the restriction operator (or boundary operator) induced by the embedding $i: X \hookrightarrow M$. If one requires that the set of operators of the form $(0.1)$ be invariant with respect to the passage to the adjoint operator, then the operator $D_{M X}$ must be the adjoint of $D_{X M}$, that is, the operator of corestriction, or, as it will be called in what follows, the coboundary operator. Thus, at first glance, our would-be theory is the theory of operators of the form

$$
\left(\begin{array}{cc}
D_{M M} & i_{*} \\
i^{*} & D_{X X}
\end{array}\right)
$$

where $i^{*}$ and $i_{*}$ are the boundary and coboundary operators and $D_{M M}$ and $D_{X X}$ are pseudodifferential operators on the corresponding manifolds. Unfortunately, operators of the form (0.2) do not form an algebra, which is a serious disadvantage from the viewpoint of elliptic theory. Indeed, the proof of the Fredholm property of an elliptic operator is carried out in the most convenient and natural form by constructing so-called regularizers, that is, almost inverses (inverses modulo compact operators). To apply this method, one must have the structure of an algebra on the set of operators and an effective calculus.

Why do not the operators (0.2) form an algebra?

A straightforward multiplication of two matrices of the form (0.2) shows that if we wish the matrices in question to form an algebra, we must first of all extend the class of (co)boundary operators. For example, instead of the (elementary) restriction operator $i^{*}$ occurring in (0.2), one should consider a general boundary operator of the form $B_{X X}^{\prime} i^{*} B_{M M}^{\prime \prime}$, and the elementary coboundary operator $i_{*}$ should be replaced by a general operator of the form $C_{M M_{*}}^{\prime} i_{X X}^{\prime \prime}$.

However, the main cause is essentially that the left upper corner of the product of two operators of this type contains an operator of entirely different structure than a pseudodifferential operator. This is not surprising, since the factors occurring in this 
operator are not pseudodifferential operators. ${ }^{1}$ Thus, it seems natural to extend the class of operators in the left upper corner so that it will include not only pseudodifferential operators, but also operators of the form

$$
D_{M M^{\prime}}^{\prime} i_{* X} D^{*} D_{M M}^{\prime \prime}
$$

(and sums of such operators), which occur in compositions. (It is remarkable that now, after an appropriate closure, the set of operators becomes closed with respect to composition, which in principle solves the problem of constructing a calculus.)

This important class of operators, which does not occur in the original statement, is the class of Green operators. These operators inevitably occur as products of boundary and coboundary operators as well as in the construction of an almost inverse operator in the Sobolev problem. Similar operators (also known as Green operators ${ }^{2}$ ) arise in the theory of boundary value problems as well as in elliptic theory on manifolds with singularities (e.g., see [Mon71, Sch91]). In the present paper, we naturally discuss only Green operators associated with the pair $(M, X)$. Green operators, as well as pseudodifferential operators, form an algebra: the product of two Green operators is itself a Green operator. In contrast with the algebra of pseudodifferential operators, the Green algebra is an algebra without unit: the identity operator is not a Green operator. One can attach the unit in the standard way and consider the inversion problem for operators of the form $1+T$, where $T$ is a Green operator, in the extended algebra. In other words, one studies the solvability of the equation

$$
(1+T) u=f
$$

for an unknown function $u$ on $M$, where $f$ is a given function on $M$. Equation (0.3) will be called the Green equation of the second kind. It is of considerable interest owing to the fact that the operator $1+T$ occurs in the ellipticity conditions for general operators associated with the pair $(M, X)$.

The algebra of operators of the form "a pseudodifferential operators plus a Green operator" is just one possible extension of the algebra of pseudodifferential operators on $M$. Such extensions arise in the presence of some additional structures on $M$ (in our case, the additional structure is the embedded submanifold $X$ ). Other examples of extensions (not covered in this survey) can be found in [SS92a, SS92b, SS94].

However, this is not the whole story. It is desirable to describe the newly introduced operators in known terms. Such a description would certainly be some kind of good luck,

\footnotetext{
${ }^{1}$ Note, however, that the similar entry in the lower right corner of the product is still a pseudodifferential operator.

${ }^{2}$ In fact, Green operators arise even in the simplest boundary value problems such as the Dirichlet problem with nonzero right-hand side $f$ for the Laplace operator in a domain. One reduces this problem to a problem with zero right-hand side by subtracting the convolution of $f$ with the Newtonian potential from the solution and then expresses the solution of the new problem as the simple layer potential corresponding to the resulting boundary conditions. The simple layer potential with density equal to the restriction to the boundary of the convolution of the right-hand side with the Newtonian potential is just the simplest Green operator applied to $f$.
} 
since it might well happen that these operators are of absolutely new nature unknown in the literature. Fortunately, this is not the case. These operators can be interpreted as Fourier integral operators on special Lagrangian manifolds. In this interpretation, each type of operators (boundary, coboundary, etc.) corresponds to its own Lagrangian manifold. These operators admit also an adequate interpretation in quite different terms in a neighborhood of the submanifold $X$. Namely, they can be represented as a special class of pseudodifferential operators acting in sections of infinite-dimensional bundles over $X$ (pseudodifferential operators with operator-valued symbols).

2. Relative elliptic theory allows one to solve an important, interesting problem in the theory of partial differential equations, known as the Sobolev problem.

To illustrate how the Sobolev problem arises, we consider simple physical examples. Suppose that we push a soap film spanning a one-dimensional contour with a thin needle. In the case of perfect nonwetting, the film collapses immediately unless we actually only touch it not trying to change its shape. In the case of perfect wetting, the needle passes through the film, whose shape remains unchanged. However, if we push a plate with the same needle, then the plate shape changes. In other words, one cannot pose "boundary conditions" for the thin film equation at a single point, but such conditions are possible for the plate equations.

Mathematically, this can be described as follows.

The function $z=z(x, y)$ describing the shape of a thin two-dimensional film spanning a closed one-dimensional contour $\{z=\varphi(x, y) \mid(x, y) \in \Gamma\}$ in the space $\mathbb{R}^{3}$ satisfies the Dirichlet problem

$$
\left\{\begin{array}{l}
\Delta z=0, \\
\left.z\right|_{\Gamma}=\varphi
\end{array}\right.
$$

in the domain $D \subset \mathbb{R}^{2}$ bounded by the plane curve $\Gamma$. Let us try to support the film with a thin needle at a point $\left(x_{0}, y_{0}, z_{0}\right) \in \mathbb{R}^{3}$ under the assumption of perfect nonwetting. Then the equation in problem (0.4) must be satisfied everywhere in $D$ except for the point $\left(x_{0}, y_{0}\right)$ :

$$
\Delta z \equiv 0 \bmod \left(x_{0}, y_{0}\right) \text {. }
$$

(This notation means that the distribution $\Delta u$ is supported at the point $\left(x_{0}, y_{0}\right)$.) Next, we must equip problem (0.4) with the additional boundary condition

$$
\left.z\right|_{\left(x_{0}, y_{0}\right)}=z_{0}
$$

By the well-known removable singularity theorem for harmonic functions, every solution of the equation (0.5) in $D$ bounded at the point $\left(x_{0}, y_{0}\right)$ (the boundedness of $z(x, y)$ follows from (0.6)) is a harmonic function everywhere in $D$ including the point $\left(x_{0}, y_{0}\right)$, so that the value $z\left(x_{0}, y_{0}\right)$ is uniquely determined by the data of problem (0.4). Hence problem (0.5)(0.6) is not solvable unless $z_{0}$ is equal to the value of the solution of problem (0.4) at that point. 
In the case of perfect wetting, the equation in problem (0.4) still holds in $D \backslash\left\{\left(x_{0}, y_{0}\right)\right\}$, but no additional conditions are imposed at the point $\left(x_{0}, y_{0}\right)$. By the removable singularity theorem, the problem

$$
\left\{\begin{array}{l}
\Delta z \equiv 0 \quad \bmod \left(x_{0}, y_{0}\right), \\
\left.z\right|_{\Gamma}=\varphi
\end{array}\right.
$$

is equivalent to problem (0.4), which means that the needle passes through the film without changing the film shape.

The situation is entirely different for the elastic plate problem

$$
\left\{\begin{array}{l}
\Delta^{2} z=0, \\
\left.z\right|_{\Gamma}=\varphi,\left.\quad \frac{\partial z}{\partial n}\right|_{\Gamma}=\psi
\end{array}\right.
$$

Here $\partial / \partial n$ is the outward normal derivative on $\Gamma$. If we replace the equation in this problem by the equation

$$
\Delta^{2} z \equiv 0 \bmod \left(x_{0}, y_{0}\right),
$$

then an additional condition of the form (0.6) is admissible, since Eq. (0.7) has a nontrivial continuous solution behaving as $r^{2} \ln r$ near $\left(x_{0}, y_{0}\right)$, where $r$ is the distance to that point.

Thus, we see that for equations of sufficiently high order one can pose problems involving "boundary conditions" on manifolds of codimension higher than one. Problems with such conditions are called Sobolev problems. The general statement of the Sobolev problem is as follows. Let $X \stackrel{i}{\hookrightarrow} M$ be a smooth embedding and $D$ an elliptic differential operator on $M$. Consider the equation

$$
D u \equiv f \bmod X,
$$

which means that $D u=f$ everywhere on the manifold $M$ except for the submanifold $X$. The problem is to equip Eq. (0.8) with well-posed boundary conditions on $X$. (Wellposedness is understood in the sense that the problem must be Fredholm.) Next, we wish to compute the index of the problem. Note that Sobolev [Sob37] was the first to consider such a problem for the polyharmonic equation; he used variational methods to prove the unique solvability of the problem in this case. The general statement and analysis of this problem, as well as the term "Sobolev problem," is due to Sternin [Ste64] (see also [Ste66]).

Note that Sobolev problems have a number of properties distinguishing them from ordinary elliptic boundary value problems for differential equations.

For example, the number of boundary conditions in these problems depends on the index of the Sobolev space in which the solution is to be sought. In particular, the Sobolev problem is essentially trivial in the space of sufficiently smooth functions, since relation (0.8) turns into an equality everywhere and no boundary conditions on $X$ are 
needed. Next, the solution of an elliptic Sobolev problem is not an infinitely smooth function in general even if the right-hand side of the equation is infinitely smooth. The solution can have singularities on the submanifold $X$.

3. Relative elliptic theory associated with the pair $(M, X)$ gives rise to remarkable relationships between elliptic pseudodifferential operators on $M$ and $X$. These relationships were studied from the topological viewpoint for the first time by Novikov and Sternin [NS66a, NS66b] and later by Sternin in the book [Ste71]. Associated with the embedding $X \stackrel{i}{\hookrightarrow} M$ is the natural pullback

$$
i_{\text {top }}^{!}: K\left(T^{*} M\right) \longrightarrow K\left(T^{*} X\right)
$$

in the topological $K$-functor, as well as the analytically defined metric trace

$$
i_{\mathrm{m}}^{*}: \operatorname{PSD}(M) \longrightarrow \operatorname{PSD}(X)
$$

and pullback

$$
i_{a}^{!}: \operatorname{PSD}(M) \longrightarrow \operatorname{PSD}(X),
$$

which take pseudodifferential operators on $M$ to pseudodifferential operators on $X$. (The first of the latter mappings is defined by the metric-dependent restriction of the symbol to the conormal bundle of the submanifold $X$, and the second is given by the formula

$$
i_{a}^{!}(D)=i^{*} D i_{*}
$$

where $i^{*}$ and $i_{*}$ are the boundary and coboundary operators.) Hence there is a natural problem of comparing the corresponding functors, i.e., establishing Riemann-Roch type theorems. It turns out that such theorems are valid indeed. The simplest theorem compares the metric trace and the topological pullback and states that the diagram

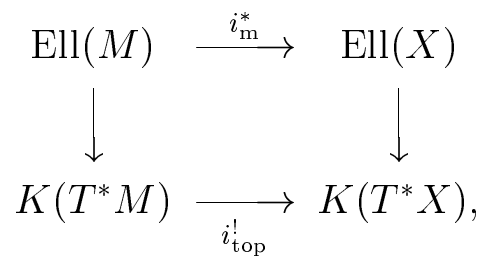

where the vertical arrows are given by the difference construction of the principal symbol, commutes. Similar theorems comparing the analytic and topological pullbacks are valid in the context of meromorphic families of elliptic operators or for the regularized pullback ${ }^{3}$

$$
i_{\text {reg }}^{!}(D)=\Delta^{z} i_{a}^{!}\left(\Delta^{-z} D\right)
$$

\footnotetext{
${ }^{3}$ Here $\Delta$ stands for an invertible operator whose principal symbol coincides with that of the Laplace operator.
} 
as $\operatorname{Re} z \rightarrow \infty$.

However, there is an important class of operators for which the "infinite" regularization is not needed. This is the class of first-order differential operators, which in particular includes all main geometric operators. Nor is the infinite regularization needed in the statement of the theorem comparing the analytic and topological pullbacks for operators whose structure in a neighborhood of $X$ is that of the exterior tensor product [AS63] of an elliptic operator on $X$ by a family of elliptic operators in the fibers of the normal bundle. This structure is also typical of geometric operators and permits one to compute the pullback for these operators explicitly.

4. Let us briefly outline the exposition in this survey. In the first part, we develop the relative theory of pseudodifferential operators as a theory associated with a smooth embedding $X \hookrightarrow M$. In the framework of this theory, we introduce the notion of ellipticity and establish the theorem on the Fredholm property. The index formula for relative elliptic operators (elliptic morphisms) is proved. By way of example, we develop the theory of Sobolev problems, prove the Fredholm property, and compute the index. Furthermore, we introduce and study the algebras of Green operators associated with the embedding $X \hookrightarrow M$, prove a criterion for the Fredholm property of the Green equation, and apply the result to the analysis of conditions for the Fredholm property of general elliptic morphisms. The second part deals with the topological aspects of elliptic theory in the category of smooth embeddings and establishes the facts described in item 3 above. As an example, we consider the Euler, Dirac, Hirzebruch, and Todd (Cauchy-Riemann) operators.

5. The present paper develops ideas, methods and results contained in [Ste64, Ste66, Ste67, Ste76, SS96, NS66a, NS66b, Ste71]. In particular, it gives a new glance on classical elliptic Sobolev problems and relative elliptic theory from the viewpoint of modern theory of differential equations. For example, boundary and coboundary operators, as well as Green operators, are interpreted in terms of Fourier integral operators.

As one can see from the cited literature, the first papers on general Sobolev problems were written about forty years ago (see [Ste64, Ste66]). Since then, quite a few remarkable papers on relative elliptic theory have been published. The notion of a coboundary operator, originally introduced in [Ste67], is nowadays widely used in general theory of differential equations. In various situations, many authors independently developed constructions close in their spirit to the constructions of relative elliptic theory (e.g., see [VE65a, VE65b, Esk73, Mon71, Sch91], etc.).

Acknowledgements. The paper was written during the authors' stay at the Institut for Mathematics, Potsdam University. The authors are grateful to Professor B.-W. Schulze for his kind hospitality. 
Conventions and notation. Let us make some remarks concerning conventions and assumptions adopted throughout the text. The dimension of the submanifold $X$ will always be denoted by $n$ and the codimension by $\nu$ (thus, $\operatorname{dim} M=n+\nu$ ). For convenience, we everywhere assume that the manifolds $M$ and $X$ are equipped with some given volume elements $d \mathrm{vol}_{M}$ and $d \mathrm{vol}_{X}$, so that one need not distinguish between functions and densities. Whenever we deal with Sobolev spaces of sections of a vector bundle, ${ }^{4}$ we assume that an Hermitian structure is given in the fibers, so the the $L^{2}$ inner product of sections is well defined and one can identify function sections and density sections. All these assumptions do not restrict the generality.

Many of our considerations are carried out in local coordinates on $X$ or $M$ in a neighborhood of some point $x_{0} \in X$. We always assume that these coordinates are chosen in the following special way. A special coordinate system on $X$ is a coordinate system $\left(x_{1}, \ldots, x_{n}\right)$ such that

$$
d \operatorname{vol}_{X}=d x_{1} \cdots d x_{n} .
$$

A coordinate system $\left(x_{1}, \ldots, x_{n}, t_{1}, \ldots, t_{\nu}\right)$ on $M$ near $X$ is called a special coordinate system if

1. the equations of the submanifold $X$ have the form

$$
t_{1}=\cdots=t_{\nu}=0
$$

2. for $t=0$, the system $\left(x_{1}, \ldots, x_{n}\right)$ is a special coordinate system on $X$;

3. one has

$$
d \operatorname{vol}_{M}=d x_{1} \cdots d x_{n} d t_{1} \cdots d t_{n}
$$

There always exists coordinate systems with these properties. We usually assume that $M$ and $X$ are Riemannian manifolds with metrics $\rho_{M}$ and $\rho_{X}$, respectively, the embedding is isometric, and the volume elements correspond to the metrics. Then one can construct a special coordinate system on $M$ as follows. Let $\pi: N X \longrightarrow X$ be the normal bundle of $X$ in $M$, i.e., the orthogonal complement of the tangent bundle $\left.T X \subset T M\right|_{X}$. We introduce local coordinates $(x, t)$ on $N X$, where $x=\left(x_{1}, \ldots, x_{n}\right)$ is a special coordinate system on $X$ in a neighborhood of $x_{0}$ and $t=\left(t_{1}, \ldots, t_{\nu}\right)$ are linear coordinates in the fiber $N_{x} X$ with respect to some orthonormal basis smoothly depending on $x \in X$ in a neighborhood of $x_{0}$. The exponential mapping

$$
\begin{aligned}
\exp : N X & \longrightarrow M, \\
\xi & \longmapsto \gamma_{\xi}(1),
\end{aligned}
$$

where $\gamma_{\xi}(\tau)$ is the geodesic on $M$ issuing from the point $\pi(\xi)$ with initial velocity vector $\dot{\gamma}_{\xi}(0)=\xi$, diffeomorphically maps some neighborhood $V$ of the zero section of the normal

\footnotetext{
${ }^{4}$ Such spaces are considered here only for complex vector bundles
} 
bundle onto a tubular neighborhood $U$ of $X$ in $M$. (In the following, we identify $U$ with $V$.) In a neighborhood of a given point $x_{0} \in X$, one can take the coordinates $(x, t)$ on $M$ transferred by the exponential mapping from $N X$. Then the submanifold $X$ is given in these coordinates by the equation $\{t=0\}$, and

$$
\rho_{M}=\rho_{X}+\sum_{j=1}^{\nu} d t_{j}^{2},\left.\quad\left[d \operatorname{vol}_{M}\right]\right|_{X}=d \operatorname{vol}_{X} d t_{1} \cdots d t_{\nu} .
$$

By multiplying $t_{1}$ by a nonzero smooth function equal to 1 for $t=0$, we ensure that the second equation holds not only on $X$, but also everywhere.

The coordinates dual to $x$ (respectively, $(x, t)$ ) in the fiber of the cotangent bundle will be denoted by $p$ (respectively, $(p, \tau)), p \in \mathbb{R}^{n}, \tau \in \mathbb{R}^{\nu}$, and the coordinates $(x, p)$ (respectively, $(x, t, p, \tau)$ ) will also be referred to as special coordinates on the cotangent bundle. For example, the symplectic forms on $T^{*} M$ and $T^{*} X$ are given by the expressions

$$
\begin{aligned}
& \omega_{M}=d p \wedge d x+d \tau \wedge d t \equiv \sum_{j=1}^{n} d p_{j} \wedge d x_{j}+\sum_{j=1}^{\nu} d \tau_{j} \wedge d t_{j} \\
& \omega_{X}=d p \wedge d x \equiv \sum_{j=1}^{n} d p_{j} \wedge d x_{j}
\end{aligned}
$$

in special coordinates.

To avoid cumbersome formulas, we also make the following simplifying assumptions, which are not important for the validity of the main results.

- All operators considered in the first part of the paper (except for the section concerning Sobolev problems) act in function spaces (the generalization to the case of operators acting in spaces of sections of vector bundles is standard).

- The manifolds $M$ and $X$ are assumed to be oriented. Moreover, we use only special coordinate systems coordinated with the orientation, so that the Riemannian volume elements $d \operatorname{vol}_{M}$ and $d \operatorname{vol}_{X}$ on $M$ and $X$ can be treated as ordinary differential forms of maximal order on these manifolds.

\section{Analytic Aspects of Relative Elliptic Theory}

Let $M$ be a smooth compact manifold without boundary and $X \hookrightarrow M$ a closed smooth submanifold. In this part, we define and study elliptic morphisms associated with the pair $(M, X)$. These morphisms act in pairs of Sobolev spaces on $M$ and $X$. The main 
tool in their construction is the technique of Fourier integral operators (briefly recalled in Appendix), and our exposition follows the standard semiclassical quantization scheme: we first introduce classical objects associated with the pair $(M, X)$ (Lagrangian manifolds with measure and symbol classes) and then describe the quantization procedure, which produces the operators that are elements of the morphism algebra, and establish its main properties, i.e., formulas for the product of operators and the adjoint operator. Next, we use these properties to state ellipticity conditions for morphisms, prove a theorem on the Fredholm property, and prove an index formula for elliptic morphisms. Finally, the results are applied to the Sobolev problem.

We note that the Lagrangian manifolds used in the construction of the morphism algebra do not satisfy the fundamental condition A.1 (see Appendix), which is assumed in the general theory of Fourier integral operators. Namely, three of these manifolds have a nonempty intersection with the zero section of at least one of the cotangent bundles in the Cartesian product of which they lie. Hence the general composition and boundedness theorems (see Subsections A.6 and A.7) are not valid for such Fourier integral operators, and we have to introduce some modifications in the statements of definitions and theorems. Moreover, one encounters three new phenomena.

1. The operators in question prove not to be continuous in the entire Sobolev scale. For example, the restriction operator

$$
i^{*}: H^{s}(M) \longrightarrow H^{s-\nu / 2}(X)
$$

is well defined and continuous only for $s>\nu / 2$ and is undefined otherwise.

2. Products of Fourier integral operators are not always well defined. For example, the product $i^{*} D i_{*}$, where $D$ is a pseudodifferential operator on $X$, is defined only for ord $D<-\nu$ (and is meaningless, say, for $D=1$ : the coboundary operator $i_{*}$ produces the delta function concentrated on $X$, whose restriction to $X$ is not defined). Hence one has to introduce restrictions on the orders of the operators to be considered.

3. One has to deal with symbol classes determined by estimates that are more complicated than the estimates (A.3), customarily used in the general theory (see Appendix). Such symbols inevitably arise from the multiplication of operators even if the symbols of the factors satisfy (A.3). For example, the product $\widehat{\Phi}=D_{X} i^{*} D_{M}$, where $D_{X}$ and $D_{M}$ are pseudodifferential operators of the form

$$
D_{X}=a\left(\frac{\partial}{\partial x}\right), \quad D_{M}=b\left(\frac{\partial}{\partial x}, \frac{\partial}{\partial t}\right)
$$

on $X$ and $M$, respectively, has the symbol $a\left(p^{\prime}\right) b\left(p^{\prime}, \tau^{\prime}\right)$ on the corresponding Lagrangian manifold. The differentiation of this symbol with respect to $p^{\prime}$ does not change the rate of growth as $\left|\tau^{\prime}\right| \rightarrow \infty$ for bounded $p^{\prime}$. 


\section{Classical objects. Lagrangian formalism}

\section{$1.1 \quad$ Lagrangian manifolds with measure}

Let

$$
i: X \hookrightarrow M
$$

be a $C^{\infty}$ embedding of a closed smooth manifold $X$ in a closed smooth manifold $M$. Associated with the pair $(M, X)$ are five remarkable $\mathbb{R}_{+}$-homogeneous Lagrangian manifolds

$$
\begin{aligned}
& L_{M}, L_{g} \subset T^{*} M \times T^{*} M, \quad L_{X} \subset T^{*} X \times T^{*} X, \\
& L_{b} \subset T^{*} X \times T^{*} M, \quad L_{c} \subset T^{*} X \times T^{*} X
\end{aligned}
$$

in Cartesian products of cotangent bundles. Furthermore, these manifolds are equipped with natural measures. All these manifolds can be obtained by the same recipe: one takes the conormal bundle of a certain submanifold in the product of bases of the corresponding cotangent bundles and reverses the sign of the momentum variables in the second factor. (Note that this change of sign automatically transforms the conormal bundle into a Lagrangian manifold with respect to the difference of symplectic forms on the first and second factors.) Let us describe the Lagrangian manifolds (1.2).

\subsubsection{The manifolds $L_{M}$ and $L_{X}$}

Invariant description. These manifolds are trivial in the sense that they are independent of the embedding and are related to $M$ and $X$ separately. They can be obtained by the above-mentioned construction from the diagonals

$$
\begin{aligned}
\Delta_{M} & =\{(\alpha, \beta) \in M \times M \mid \alpha=\beta\} \subset M \times M, \\
\Delta_{X} & =\{(\alpha, \beta) \in X \times X \mid \alpha=\beta\} \subset X \times X
\end{aligned}
$$

in $M \times M$ and $X \times X$, respectively. Thus,

$$
L_{M}=\tilde{N}^{*}\left(\Delta_{M}\right) \subset T^{*} M \times T^{*} M, \quad L_{X}=\tilde{N}^{*}\left(\Delta_{X}\right) \subset T^{*} X \times T^{*} X,
$$

Here $N^{*}(Y)$ is the conormal bundle of $Y$ and the tilde on $N^{*}$ stands for reversing the sign of the cotangent variable in the second factor $T^{*} M$ ( or $\left.T^{*} X\right)$. One can readily see that the manifolds $L_{M}$ and $L_{X}$ themselves are diagonals in products of cotangent bundles, i.e., the graphs of identity canonical transformations:

$$
L_{M}=\Delta_{T^{*} M}, \quad L_{X}=\Delta_{T^{*} X} .
$$

The manifolds $L_{M}$ and $L_{X}$ bear the natural measures $\mu_{M}$ and $\mu_{X}$ given by the formulas

$$
\mu_{M}=\frac{\pi_{j}^{*}\left(\omega_{M}^{\wedge(n+\nu)}\right)}{(n+\nu) !}, \quad \mu_{X}=\frac{\pi_{j}^{*}\left(\omega_{X}^{\wedge n}\right)}{n !}
$$


where $\pi_{j}$ is the projection on the $j$ th factor in the corresponding product of cotangent bundles. (These formulas are the standard formulas defining measures on graphs of canonical transformations [MSS90]. By virtue of the Lagrangian property, the result is independent of the choice of $j=1,2$.)

Coordinate description. The manifolds $L_{M}$ and $L_{X}$ are given in special coordinate systems on the cotangent bundles by the formulas

$$
\begin{aligned}
L_{M} & =\left\{\left(x, t, p, \tau, x^{\prime}, p^{\prime}, t^{\prime}, \tau^{\prime}\right) \mid x=x^{\prime}, t=t^{\prime}, p=p^{\prime}, \tau=\tau^{\prime}\right\}, \\
L_{X} & =\left\{\left(x, p, x^{\prime}, p^{\prime}\right) \mid x=x^{\prime}, p=p^{\prime}\right\} .
\end{aligned}
$$

(From now on, the coordinates on the second factor in the product of cotangent bundles are indicate by primes.) The canonical coordinates (see Subsection A.2) on $L_{M}$ and $L_{X}$ can be taken in the form $\left(x, t, p^{\prime}, \tau^{\prime}\right)$ and $\left(x, p^{\prime}\right)$, respectively. Then the measures are given by the formulas

$$
\begin{aligned}
& \mu_{M}=d p_{1}^{\prime} \wedge d x_{1} \wedge \cdots \wedge d p_{n}^{\prime} \wedge d x_{n} \wedge d \tau_{1}^{\prime} \wedge d t_{1} \wedge \cdots \wedge d \tau_{\nu}^{\prime} \wedge d t_{\nu} \\
& \mu_{X}=d p_{1}^{\prime} \wedge d x_{1} \wedge \cdots \wedge d p_{n}^{\prime} \wedge d x_{n}
\end{aligned}
$$

and the generating functions of these Lagrangian manifolds (see Subsection A.2) have the form

$$
S_{M}\left(x, t, p^{\prime}, \tau^{\prime}\right)=p^{\prime} x+\tau^{\prime} t, \quad S_{X}\left(x, p^{\prime}\right)=p^{\prime} x
$$

\subsubsection{The manifold $L_{b}$}

Invariant description. This manifold can be obtained by applying the above-mentioned construction to the graph of the embedding $i$ :

$$
L_{b}=\widetilde{N}^{*}(\text { graph } i) \subset T^{*} X \times T^{*} M .
$$

The manifold $L_{b}$ (as well as all Lagrangian manifolds considered below) is not the graph of any canonical transformation, and so we cannot apply the standard construction (1.4) of the measure. To obtain a natural measure on $L_{b}$, consider the mappings

$$
\pi_{1}: L_{b} \longrightarrow T^{*} X, \quad \pi_{2}: L_{b} \longrightarrow T^{*} M
$$

obtained by the restriction to $L_{b}$ of the natural projections on the first and second factors, respectively, in the Cartesian product $T^{*} X \times T^{*} M$. Let $\alpha \in L_{b}$ be an arbitrary point. The differentials

$$
\pi_{1 *}: T_{\alpha} L_{b} \longrightarrow T_{\pi_{1}(\alpha)} T^{*} X, \quad \pi_{2 *}: T_{\alpha} L_{b} \longrightarrow T_{\pi_{2}(\alpha)} T^{*} M
$$

possess the following properties: 
1. $\pi_{1 *}$ is an epimorphism;

2. $\left.\pi_{2 *}\right|_{\operatorname{ker} \pi_{1 *}}$ is an isomorphism of the kernel ker $\pi_{1 *}$ onto the subspace in $T_{\pi_{2}(\alpha)} T^{*} M$ parallel to the fiber $N_{\pi\left(\pi_{1}(\alpha)\right)}^{*} X$ of $N^{*} X$ at the point $\pi\left(\pi_{1}(\alpha)\right)$ (where $\pi: T^{*} X \longrightarrow X$ is the natural projection).

After natural identifications, we obtain the exact sequence of vector spaces

$$
0 \longrightarrow N_{\pi\left(\pi_{1}(\alpha)\right)}^{*} X \longrightarrow T_{\alpha} L_{b} \longrightarrow T_{\pi_{1}(\alpha)} T^{*} X \longrightarrow 0 .
$$

There are natural volume forms on the fibers of $T T^{*} X$ (namely, the normalized power

$$
d \operatorname{vol}_{T^{*} X}=\frac{1}{n !}\left(\omega_{X}\right)^{\wedge n}
$$

of the symplectic structure on $X$ ) as well as on the fibers of $N^{*} X$ (namely, the form

$$
d \operatorname{vol}_{N^{*} X}=d \tau_{1} \wedge \ldots \wedge \tau_{\nu}
$$

where $\tau_{1}, \ldots, \tau_{\nu}$ are the momentum variables dual to the coordinates $t_{1}, \ldots, t_{\nu}$ from some special coordinate system $(x, t)$; the form on the right-hand side in (1.10) is independent of the choice of coordinates). We define a volume form $\mu_{b}$ on $T_{\alpha} L_{b}$ by arbitrarily splitting the sequence (1.8) and by setting

$$
\mu_{b}=d \operatorname{vol}_{T^{*} X} \wedge d \operatorname{vol}_{N^{*} X}
$$

(The result is independent of the splitting.)

Thus, we have defined a measure $\mu_{b}$ on the manifold $L_{b}$.

Coordinate description. The manifold $L_{b}$ is given in special coordinate systems on the cotangent bundles by the formulas

$$
L_{b}=\left\{\left(x, p, x^{\prime}, t^{\prime}, p^{\prime}, \tau^{\prime}\right) \mid x=x^{\prime}, t^{\prime}=0, p=p^{\prime}\right\} .
$$

The canonical coordinates on $L_{b}$ can be taken conveniently in the form $\left(x, p^{\prime}, \tau^{\prime}\right)$. Then the measure is given by the formula

$$
\mu_{b}=d p_{1}^{\prime} \wedge d x_{1} \wedge \cdots \wedge d p_{n}^{\prime} \wedge d x_{n} \wedge d \tau_{1}^{\prime} \wedge \cdots \wedge d \tau_{\nu}^{\prime},
$$

and the generating functions of $L_{b}$ has the form

$$
S_{b}\left(x, p^{\prime}, \tau^{\prime}\right)=p^{\prime} x .
$$




\subsubsection{The manifold $L_{c}$}

Invariant description. This manifold is defined as

$$
L_{c}=\tilde{N}^{*}\left({ }^{T} \operatorname{graph} i\right) \subset T^{*} M \times T^{*} X,
$$

where the submanifold ${ }^{T}$ graph $i \subset M \times X$ is obtained as the transpose of graph $i$. (The factors in the Cartesian product are transposed.) Clearly, $L_{c}$ itself coincides with $L_{b}$ up to transposition:

$$
L_{c}={ }^{T} L_{b} \subset T^{*} M \times T^{*} X
$$

More formally, consider the transposition isomorphism

$$
\begin{aligned}
\varkappa: T^{*} X \times T^{*} M & \longrightarrow T^{*} M \times T^{*} X, \\
(\alpha, \beta) & \longmapsto(\beta, \alpha) ;
\end{aligned}
$$

then

$$
\varkappa\left(L_{b}\right)=L_{c} .
$$

We use the isomorphism (1.14) and define a measure on $L_{c}$ by setting

$$
\mu_{c}=\left(\varkappa^{-1}\right)^{*}\left(\mu_{b}\right) .
$$

Coordinate description. The manifold $L_{c}$ is given in special coordinate systems on the cotangent bundles by the formula

$$
L_{c}=\left\{\left(x, t, p, \tau, x^{\prime}, p^{\prime}\right) \mid x=x^{\prime}, t=0, p=p^{\prime}\right\} .
$$

The canonical coordinates on $L_{c}$ can be taken in the form $\left(x, \tau, p^{\prime}\right)$. Then the measure is given by the formula

$$
\mu_{c}=d p_{1}^{\prime} \wedge d x_{1} \wedge \cdots \wedge d p_{n}^{\prime} \wedge d x_{n} \wedge d \tau_{1} \wedge \cdots \wedge d \tau_{\nu}
$$

and the generating function of $L_{b}$ has the form

$$
S_{b}\left(x, \tau, p^{\prime}\right)=p^{\prime} x .
$$

\subsubsection{The manifold $L_{g}$}

Invariant description. This Lagrangian manifold is defined as

$$
L_{g}=\tilde{N}^{*}(W) \subset T^{*} M \times T^{*} M,
$$

where

$$
W=\{(\alpha, \alpha) \in M \times M \mid \alpha \in X\} .
$$


Let

$$
\pi_{1,2}:\left.L_{g} \longrightarrow T^{*} M\right|_{X} \subset T^{*} M
$$

be the mappings obtained by the restriction to $L_{g}$ of the natural projections of $T^{*} M \times T^{*} M$ on the first and second factors. Consider the mapping

$$
p: L_{g} \longrightarrow T^{*} X
$$

given by the composition $p=\tilde{\pi} \circ \pi_{1}$, where

$$
\tilde{\pi}:\left.T^{*} M\right|_{X} \longrightarrow T^{*} X
$$

is the natural projection. (Note that $\tilde{\pi} \circ \pi_{1}=\tilde{\pi} \circ \pi_{2}$.) Next, $p_{*}$ is an epimorphism at an arbitrary point $\alpha \in L_{g}$, and the mapping

$$
\left.\left(\pi_{1 *} \oplus \pi_{2 *}\right)\right|_{\text {ker } p_{*}}
$$

is an isomorphism of ker $p_{*}$ onto the direct sum of two copies of the tangent space to a fiber of the conormal bundle of $X$. Thus, we obtain the exact sequence

$$
0 \longrightarrow N_{\pi(p(\alpha))}^{*} X \oplus N_{\pi(p(\alpha))}^{*} X \longrightarrow T_{\alpha} L_{g} \longrightarrow T_{p(\alpha)} T^{*} X \longrightarrow 0
$$

for an arbitrary point $\alpha \in L_{g}$ (where $\pi: T^{*} \longrightarrow X$ is the natural projection). This sequence permits one to define a measure $\mu_{g}$ on $L_{g}$ by the formula

$$
\mu_{g}=d \operatorname{vol}_{N^{*} X} \wedge d \operatorname{vol}_{N^{*} X} \wedge d \operatorname{vol}_{T^{*} X},
$$

where the factors on the right-hand side are given by formulas (1.9) and (1.10). (The two factors $d \operatorname{vol}_{N^{*} X}$ correspond to the two copies of $N_{\pi(p(\alpha))}^{*} X$ in the expansion (1.22).)

Coordinate description. The manifold $L_{g}$ is given in special coordinate systems on the cotangent bundles by the formulas

$$
L_{g}=\left\{\left(x, t, p, \tau, x^{\prime}, t^{\prime}, p^{\prime}, \tau^{\prime}\right) \mid x=x^{\prime}, t=t^{\prime}=0, p=p^{\prime}\right\} .
$$

The canonical coordinates on $L_{g}$ can be taken in the form $\left(x, \tau, p^{\prime}, \tau^{\prime}\right)$. Then the measure is given by the formula

$$
\mu_{g}=d p_{1}^{\prime} \wedge d x_{1} \wedge \cdots \wedge d p_{n}^{\prime} \wedge d x_{n} \wedge d \tau_{1} \wedge \cdots \wedge d \tau_{\nu} \wedge d \tau_{1}^{\prime} \wedge \cdots \wedge d \tau_{\nu}^{\prime}
$$

and the generating functions of $L_{g}$ has the form

$$
S_{b}\left(x, \tau, p^{\prime}, \tau^{\prime}\right)=p^{\prime} x .
$$

Thus, we have defined the Lagrangian manifolds (1.2), constructed some natural measures on these manifolds, and described canonical coordinates related to special coordinate systems in the cotangent bundles. (In the following, these canonical coordinates on Lagrangian manifolds will often be referred to as special canonical coordinates.) The following assertion holds. 
Proposition 1.1. The measures $\mu_{M}, \mu_{X}, \mu_{b}, \mu_{c}$, and $\mu_{g}$ are quantized.

(As to the notion of quantized measures on Lagrangian manifolds, e.g., see [MSS90].) We omit the proof.

Thus, Maslov's canonical operator, which is used in the construction of Fourier integral operators (see Appendix), is well defined on all above-mentioned Lagrangian manifolds with measure.

We should possibly comment on the notation. The manifolds $L_{X}$ and $L_{M}$ are the graphs of identity canonical transformations. (The corresponding Fourier integral operators are just pseudodifferential operators.) The subscripts $b, c$, and $g$ on the manifolds $L_{b}$, $L_{g}$, and $L_{c}$ have been chosen in view of the fact that the corresponding Fourier integral operators are known as boundary operators, coboundary operators, and Green operators, respectively.

\subsection{Composition and transposition formulas}

Now we shall study compositions and transpositions of the Lagrangian manifolds introduced above. This proves useful, since the composition of Lagrangian manifolds corresponds to the composition of the corresponding Fourier integral operators and the transposition corresponds to the passage to the adjoint operator. The general definition of composition for Lagrangian manifolds is given in Appendix, Subsection A.3. We have already noted that three of our Lagrangian manifolds, i.e., $L_{b}, L_{c}$, and $L_{g}$, fail to satisfy the fundamental condition A.1: each of these manifolds has a nonempty intersection with the zero section of at least one of the cotangent bundles forming the product where this manifold lies. Hence the composition need not be proper in general (cf. Subsection A.3), and we have to modify the definition.

\subsubsection{Definition of the composition}

Definition 1.2. Let

$$
L_{1} \subset T^{*} M_{1} \times T^{*} M_{2}, \quad L_{2} \subset T^{*} M_{2} \times T^{*} M_{3}
$$

be two Lagrangian manifolds. Suppose that the following conditions hold.

1. The composition $L=L_{1} \circ L_{2}$ given by (A.2)) is clean and connected (see Subsection A.3).

2. The projection

$$
p: \widetilde{L} \longrightarrow L
$$

where

$$
\widetilde{L}=\left(L_{1} \times L_{2}\right) \cap\left(T^{*} M_{1} \times \Delta_{T^{*} M_{2}} \times T^{*} M_{3}\right),
$$


is a locally trivial bundle over the manifold $L$. (The fiber of this bundle over a point $(\alpha, \beta) \in L$ will be denoted by $F_{(\alpha, \beta)}$; the dimension will be denoted by $\operatorname{dim} F_{(\alpha, \beta)}=e$ and referred to as the excess of the composition, just as in the general theory.)

Then we say that the composition $L=L_{1} \circ L_{2}$ is well defined. (Note that $L$ is automatically a Lagrangian manifold.)

Needless to say, if the projection (1.26) is proper, then the local triviality of the bundle (1.26) follows from Thom's theorem, and we return to the standard theory.

\subsubsection{The composition table}

Now we are in a position to state the composition theorem for Lagrangian manifolds.

Theorem 1.3. Every meaningful composition of two Lagrangian manifolds from the list (1.2) is well defined. The composition table has the following form (the first factor corresponds to a row and the second, to a column):

\begin{tabular}{c|c|c|c|c|c|} 
& $L_{X}$ & $L_{M}$ & $L_{b}$ & $L_{c}$ & $L_{g}$ \\
\hline$L_{X}$ & $L_{X}$ & - & $L_{b}$ & - & - \\
\hline$L_{M}$ & - & $L_{M}$ & - & $L_{c}$ & $L_{g}$ \\
\hline$L_{b}$ & - & $L_{b}$ & - & $L_{X}$ & $L_{b}$ \\
\hline$L_{c}$ & $L_{c}$ & - & $L_{g}$ & - & - \\
\hline$L_{g}$ & - & $L_{g}$ & - & $L_{c}$ & $L_{g}$ \\
\hline
\end{tabular}

Here dashes are placeholders for meaningless compositions.

Next, the fiber of the projection (1.26) is a singleton $(e=0)$ for all compositions except for the four compositions in the intersection of the last three rows and the last three columns. For any of these four compositions, the fiber of the projection (1.26) is naturally isomorphic to the fiber of the normal bundle $N^{*} X$ over the corresponding point of $X$ (thus, $e=\nu$ in these cases).

Proof. The proof goes by a straightforward computation.

Remark 1.4. We see that for the four compositions mentioned in the theorem (which will be referred to as exceptional), the fiber of the projection (1.26) is not compact. (It is isomorphic to $\mathbb{R}^{\nu}$.) Since the formula for the symbol of the product of Fourier integral operators (see Subsections 1.4 and 2.2) contains integration over the fiber, it is not at all surprising that order restrictions guaranteeing the convergence of the integral arise for such products. 


\subsubsection{Transposition}

The behavior of our Lagrangian manifolds and measures under transposition is described by the following assertion, which can readily be verified by a straightforward computation.

Proposition 1.5. One has

$$
{ }^{T} L_{X}=L_{X}, \quad{ }^{T} L_{M}=L_{M}, \quad{ }^{T} L_{b}=L_{c}, \quad{ }^{T} L_{c}=L_{b}, \quad{ }^{T} L_{g}=L_{g} .
$$

Moreover, the transposition takes the above-defined measure on any of these Lagrangian manifolds to the corresponding measure on the transpose (neglecting the sign).

\subsection{Symbols}

Now we shall introduce symbol spaces on each of the Lagrangian manifolds (1.2). These symbol spaces will be used in the quantization procedure (i.e., in the construction of the corresponding Fourier integral operators). Just as in the standard theory of pseudodifferential operators (or Fourier integral operators), there are various approaches to the definition of symbol spaces. On the one hand, one can define a symbol as an arbitrary function satisfying certain estimates (which guarantee the continuity of the corresponding operator in appropriate Sobolev spaces). In this case, the notion of principal symbol (which alone is meaningful in classical mechanics) becomes rather complicated and inconvenient: one has to define the principal symbol as an element of a quotient space of the symbol space. On the other hand, one can single out a narrower space of classical symbols asymptotic for large values of the momentum variables to some $\mathbb{R}_{+}$-homogeneous function of these variables. It is the latter function that plays the role of the principal symbol. (Note that it usually does not lie in the original symbol space, since it has a singularity at zero. Thus, one has to use cutoff functions in the quantization procedure.) It is most convenient to combine the two approaches: one first introduces a wider symbol class and proves boundedness and composition theorems and then observes the invariance of the space of operators with classical symbols with respect to composition and studies ellipticity, the Fredholm property, etc. in this narrower class.

In this section, we introduce symbol spaces using the above-mentioned approach. All main statements are given in special coordinates and special canonical coordinates on the Lagrangian manifolds, for we believe that coordinate statements are easier to comprehend than invariant statements in this case. We omit the proof of independence of our definitions of the choice of special local coordinates. Needless to say, one can also give an invariant description of symbol spaces. By way of example, we give such a description for the manifolds $L_{b}$ and $L_{g}$.

Finally, we note that the traditional approach to the definition of classical symbols requires that the symbols have asymptotic series expansions in homogeneous functions at infinity. The operators to be constructed here act only in a limited interval of the Sobolev scale, and hence there is no need to require the existence of "long" expansions. 
Furthermore, we deal with qualitative issues of elliptic theory, where lower-order terms play essentially no role. Therefore, we define classical symbols imposing only the minimal requirement of existence of only one (leading) term of the asymptotic expansion.

Throughout the following, by $\chi(r), r \in \mathbb{R}_{+}$, we denote a smooth nonnegative function such that

$$
\chi(r)= \begin{cases}0, & r \leq 1 / 2, \\ 1, & r \geq 1\end{cases}
$$

\subsubsection{Symbols on $L_{M}$ and $L_{X}$}

These symbols are the symbols of pseudodifferential operators. We briefly recall the well-known spaces of such symbols so as to set the notation and clarify the scheme of the subsequent reasoning by a well-known example. To be definite, we consider the Lagrangian manifold $L_{X}$. The notation for $L_{M}$ is entirely similar.

Definition 1.6. By $S^{m}\left(L_{X}\right)$, where $m \in \mathbb{R}$, we denote the space of smooth functions $a$ on the manifold $L_{X}$ such that the following estimates hold in the canonical coordinates:

$$
\left|\frac{\partial^{\alpha+\beta} a\left(x, p^{\prime}\right)}{\partial x^{\alpha} \partial p^{\prime \beta}}\right| \leq C_{\alpha \beta}\left(1+\left|p^{\prime}\right|\right)^{m-|\beta|}, \quad|\alpha|+|\beta|=0,1,2, \ldots
$$

By $O^{m}\left(L_{X}\right)$ we denote the space of functions $a$ on the manifold $L_{X}$ such that $a$ is smooth outside the zero section $\left(\left|p^{\prime}\right|=0\right)$ and $\mathbb{R}_{+}$-homogeneous of degree $m$ (with respect to the standard action of the group $\mathbb{R}_{+}$in the fibers).

By $S_{c l}^{m}\left(L_{X}\right) \subset S^{m}\left(L_{X}\right)$ we denote the subspace of functions representable in the form

$$
a=\chi\left(\left|p^{\prime}\right|\right) a_{0}+b, \quad a_{0} \in O^{m}\left(L_{X}\right), \quad b \in S^{m-\varepsilon}\left(L_{X}\right) \text { for some } \varepsilon>0 .
$$

Elements of the spaces $S^{m}\left(L_{X}\right), S_{c l}^{m}\left(L_{X}\right)$, and $O^{m}\left(L_{X}\right)$ are called, respectively, symbols, classical symbols, and homogeneous (principal) symbols of order $m$ on $L_{X}$. The element $a_{0} \in O^{m}\left(L_{X}\right)$ corresponding to a symbol $a \in S_{c l}^{m}\left(L_{X}\right)$ according to (1.29) is called the (homogeneous) principal part of $a$.

One can readily see that the homogeneous principal part of a symbol is uniquely determined.

The definitions of homogeneous principal symbols for the other Lagrangian manifolds look somewhat more complicated, since these symbols have singularities not only on the zero section. 


\subsubsection{Symbols on $L_{b}$ and $L_{c}$}

First, we introduce symbol spaces on the manifold $L_{b}$. The symbol spaces on $L_{c}$ are obtained from these via the mapping $\left(\varkappa^{-1}\right)^{*}$, where $\varkappa$ is the isomorphism (1.14).

Let $k$ and $l$ be given real numbers.

Definition 1.7. By $S_{b}^{k, l}\left(L_{b}\right)$ we denote the space of smooth functions $a$ on the manifold $L_{b}$ such that the following estimates hold in the canonical coordinates:

$$
\begin{gathered}
\left|\frac{\partial^{\alpha+\beta+\gamma} a\left(x, p^{\prime}, \tau^{\prime}\right)}{\partial x^{\alpha} \partial p^{\prime \beta} \partial \tau^{\prime \gamma}}\right| \leq C_{\alpha \beta \gamma}\left(1+\left|p^{\prime}\right|\right)^{k-|\beta|}\left(1+\left|p^{\prime}\right|+\left|\tau^{\prime}\right|\right)^{l-|\gamma|}, \\
|\alpha|+|\beta|+|\gamma|=0,1,2, \ldots
\end{gathered}
$$

By $O_{b}^{k, l}\left(L_{b}\right)$ we denote the space of functions $a$ on the manifold $L_{b}$ with the following properties:

1. $a$ is defined and infinitely smooth outside the set

$$
\Gamma=\left\{p^{\prime}=0\right\} \subset L_{b}
$$

2. $a$ is $\mathbb{R}_{+}$-homogeneous of degree $k+l$ in the variables $\left(p, \tau^{\prime}\right)$ :

$$
a\left(x, \lambda p^{\prime}, \lambda \tau^{\prime}\right)=\lambda^{k+l} a\left(x, p^{\prime}, \tau^{\prime}\right), \quad \lambda \in \mathbb{R}_{+}, \quad p^{\prime} \neq 0
$$

3. for $p^{\prime} \neq 0$, the function $a$ satisfies the estimates

$$
\begin{gathered}
\left|\frac{\partial^{\alpha+\beta+\gamma} a\left(x, p^{\prime}, \tau^{\prime}\right)}{\partial x^{\alpha} \partial p^{\prime \beta} \partial \tau^{\prime \gamma}}\right| \leq C_{\alpha \beta \gamma}\left|p^{\prime}\right|^{k-|\beta|}\left(\left|p^{\prime}\right|+\left|\tau^{\prime}\right|\right)^{l-|\gamma|}, \\
|\alpha|+|\beta|+|\gamma|=0,1,2, \ldots
\end{gathered}
$$

By $S_{b, c l}^{k, l}\left(L_{b}\right) \subset S_{b}^{k, l}\left(L_{b}\right)$ we denote the subspace of functions representable in the form

$$
\begin{gathered}
a=\chi\left(\left|p^{\prime}\right|\right) a_{0}+b \\
a_{0} \in O_{b}^{k, l}\left(L_{b}\right), \quad b \in S_{b}^{k-\varepsilon, l}\left(L_{b}\right) \oplus S_{b}^{k, l-\varepsilon}\left(L_{b}\right) \text { for some } \varepsilon>0 .
\end{gathered}
$$

Elements of the spaces $S_{b}^{k, l}\left(L_{b}\right), S_{b, c l}^{k, l}\left(L_{b}\right)$, and $O_{b}^{k, l}\left(L_{b}\right)$ are called, respectively, symbols, classical symbols, and homogeneous (principal) symbols of type $(k, l)$ on $L_{b}$. The element $a_{0} \in O_{b}^{k, l}\left(L_{b}\right)$ corresponding to a symbol $a \in S_{b, c l}^{k, l}\left(L_{b}\right)$ according to (1.31) is called the (homogeneous) principal part of $a$. The number $r=k+l+\nu / 2$ is called the order of $a$ (or $a_{0}$ ) and is denoted by

$$
\operatorname{ord} a=\operatorname{ord} a_{0}=k+l+\nu / 2 .
$$

This is well defined, as shown by the following almost obvious proposition. (The proof is by straightforward computation and is therefore omitted.) 
Proposition 1.8. Let $a \in O_{b}^{k, l}\left(L_{b}\right)$. Then $\chi\left(\left|p^{\prime}\right|\right) a \in S_{b, c l}^{k, l}\left(L_{b}\right)$. If $\chi_{1}(r)$ is another smooth function with property (1.28), then

$$
\left(\chi\left(\left|p^{\prime}\right|\right)-\chi_{1}\left(\left|p^{\prime}\right|\right)\right) a \in S_{b}^{k-1, l}\left(L_{b}\right) .
$$

It follows from the definition of the space $O_{b}^{k, l}\left(L_{b}\right)$ and $S_{b, c l}^{k, l}\left(L_{b}\right)$ that the function $a_{0}$ in (1.31) is determined for a given $a \in S_{b, c l}^{k, l}\left(L_{b}\right)$ uniquely and is independent of the choice of the cutoff function $\chi$.

Invariant description. Let us show how one can rewrite the estimates (1.30) in invariant form. To this end, we note that $L_{b}$ is a vector bundle; we define a function $|\alpha|$, $\alpha \in L_{b}$, as a norm in the fibers smoothly depending on the point of the base. In a similar way, we introduce a norm in the fibers of $T^{*} X$.

We consider two kinds of vector fields on $L_{b}$ :

- vector fields annihilated by $\pi_{1 *}$, where $\pi_{1}$ is the projection on the first factor in the product $T^{*} X \times T^{*} M$; such fields will be referred to as normal to $T^{*} X$;

- vector fields taken by $\pi_{1 *}$ to well-defined vector fields on $T^{*} X$ (thus, for each $\beta \in$ $T^{*} X$ the application of $\pi_{1 *}$ to the vector of the field at a point $\alpha \in \pi_{1}^{-1}(\beta)$ gives a result independent of the choice of $\alpha$ ); such fields will be referred to as lifted from $T^{*} X$.

Now we can restate the first part of Definition 1.7 as follows.

Definition 1.9. By $S_{b}^{k, l}\left(L_{b}\right)$, where $k, l \in \mathbb{R}$, we denote the space of smooth functions $a$ on the manifold $L_{b}$ such that the following condition holds: suppose that $\left\{V_{1}, \ldots, V_{j}\right\}$ is an arbitrary (possibly, empty) finite set of smooth vector fields on $L_{b}$ such that each of the fields is homogeneous of degree 0 or 1 and is either normal to $T^{*} X$ or lifted from $T^{*} X$; then

$$
\left|V_{1} \cdots V_{j} a(\alpha)\right| \leq C(1+|\alpha|)^{l-j_{1}}\left(1+\left|\pi_{1}(\alpha)\right|\right)^{k-j_{2}},
$$

where $j_{1}$ is the number of first-degree homogeneous fields normal to $T^{*} X$ and $j_{2}$ is the number of first-degree homogeneous fields lifted from $T^{*} X$. (The constant $C$ in (1.32) depends on the choice of the fields $V_{1}, \ldots, V_{j}$.)

Symbols on $L_{c}$. We define symbol spaces on $L_{c}$ as follows.

Definition 1.10. The spaces

$$
\begin{gathered}
S_{c}^{m, k}\left(L_{c}\right)=\left(\varkappa^{-1}\right)^{*} S_{b}^{k, m}\left(L_{b}\right), \quad O_{c}^{m, k}\left(L_{c}\right)=\left(\varkappa^{-1}\right)^{*} O_{b}^{k, m}\left(L_{b}\right), \\
S_{c, c l}^{m, k}\left(L_{c}\right)=\left(\varkappa^{-1}\right)^{*} S_{b, c l}^{k, m}\left(L_{b}\right),
\end{gathered}
$$


where $\varkappa$ is the diffeomorphism (1.14), are called, respectively, the spaces of symbols, classical symbols, and homogeneous (principal) symbols of type $(m, k)$ on $L_{c}$. The element $a_{0} \in O_{c}^{m, k}\left(L_{c}\right)$ corresponding to a symbol $a \in S_{c, c l}^{m, k}\left(L_{c}\right)$ according to the formula

$$
a=\chi\left(\left|p^{\prime}\right|\right) a_{0}+b, \quad b \in S_{c}^{m-\varepsilon, k}\left(L_{c}\right) \oplus S_{c}^{m, k-\varepsilon}\left(L_{c}\right) \text { for some } \varepsilon>0,
$$

is called the (homogeneous) principal part of $a$. The number $r=m+k+\nu / 2$ is called the order of $a$ (or $\left.a_{0}\right)$ and is denoted by ord $a$.

Remark 1.11. The "correction" $\nu / 2$ in the definition of the order of symbols on $L_{b}$ and $L_{c}$ ensures that the order of a symbol is equal to the order in the Sobolev scale of the operator obtained by the quantization of the symbol. The correction $\nu$ in the definition of the order of symbols on $L_{g}$ has the same meaning.

\subsubsection{Symbols on $L_{g}$}

Let $m, k$, and $l$ be given real numbers.

Definition 1.12. By $S_{g}^{m, k, l}\left(L_{g}\right)$ we denote the space of smooth functions $a$ on the manifold $L_{g}$ such that the following estimates hold in the canonical coordinates:

$$
\begin{aligned}
& \left|\frac{\partial^{\alpha+\beta+\gamma+\delta} a\left(x, p^{\prime}, \tau, \tau^{\prime}\right)}{\partial x^{\alpha} \partial p^{\prime \beta} \partial \tau^{\gamma} \partial \tau^{\prime \delta}}\right| \\
& \leq C_{\alpha \beta \gamma \delta}\left(1+\left|p^{\prime}\right|\right)^{k-|\beta|}\left(1+\left|p^{\prime}\right|+|\tau|\right)^{m-|\gamma|}\left(1+\left|p^{\prime}\right|+\left|\tau^{\prime}\right|\right)^{l-|\delta|}, \\
& |\alpha|+|\beta|+|\gamma|+|\delta|=0,1,2, \ldots .
\end{aligned}
$$

By $O_{g}^{m, k, l}\left(L_{g}\right)$ we denote the space of functions $a$ on the manifold $L_{g}$ with the following properties:

1. $a$ is defined and infinitely smooth outside the set

$$
\Gamma=\left\{p^{\prime}=0\right\} \subset L_{g}
$$

2. $a$ is $\mathbb{R}_{+}$-homogeneous of degree $m+k+l$ in the variables $\left(p^{\prime}, \tau, \tau^{\prime}\right)$ :

$$
a\left(x, \lambda p^{\prime}, \lambda \tau, \lambda \tau^{\prime}\right)=\lambda^{k+l} a\left(x, p^{\prime}, \tau, \tau^{\prime}\right), \quad \lambda \in \mathbb{R}_{+}, \quad p^{\prime} \neq 0
$$

3. For $p \neq 0$, the function $a$ satisfies the estimates

$$
\left|\frac{\partial^{\alpha+\beta+\gamma+\delta} a\left(x, p^{\prime}, \tau, \tau^{\prime}\right)}{\partial x^{\alpha} \partial p^{\prime \beta} \partial \tau^{\gamma} \partial \tau^{\prime \delta}}\right| \leq C_{\alpha \beta \gamma \delta}\left|p^{\prime}\right|^{k-|\beta|}\left(\left|p^{\prime}\right|+|\tau|\right)^{m-|\gamma|}\left(\left|p^{\prime}\right|+\left|\tau^{\prime}\right|\right)^{l-|\delta|},
$$

for $|\alpha|+|\beta|+|\gamma|+|\delta|=0,1,2, \ldots$ 

form

By $S_{g, c l}^{m, k, l}\left(L_{g}\right) \subset S_{g}^{m, k, l}\left(L_{g}\right)$ we denote the subspace of functions representable in the

$$
\begin{gathered}
a=\chi\left(\left|p^{\prime}\right|\right) a_{0}+b, \quad a_{0} \in O_{g}^{m, k, l}\left(L_{g}\right), \\
b \in S_{g}^{m-\varepsilon, k, l}\left(L_{g}\right) \oplus S_{g}^{m, k-\varepsilon, l}\left(L_{g}\right) \oplus S_{g}^{m, k, l-\varepsilon}\left(L_{g}\right) \text { for some } \varepsilon>0 .
\end{gathered}
$$

Elements of the spaces $S_{g}^{m, k, l}\left(L_{g}\right), S_{g, c l}^{m, k, l}\left(L_{g}\right)$, and $O_{g}^{m, k, l}\left(L_{g}\right)$ are called, respectively, symbols, classical symbols, and homogeneous (principal) symbols of type $(m, k, l)$ on $L_{g}$. The element $a_{0} \in O_{g}^{m, k, l}\left(L_{g}\right)$ corresponding to a symbol $a \in S_{g, c l}^{m, k, l}\left(L_{g}\right)$ according to (1.35) is called the (homogeneous) principal part of the symbol $a$. The number $r=m+k+l+\nu$ is called the order of $a$ (or $a_{0}$ ) and is denoted by

$$
\text { ord } a=\operatorname{ord} a_{0}=m+k+l+\nu .
$$

Just as in the other cases, one can verify that the principal part of a symbol is well defined and is unique.

Invariant description. Let us show how one can rewrite the estimates (1.34) in invariant form. To this end, we consider the following three kinds of vector fields on $L_{g}$ :

- vector fields everywhere tangent to the first copy of $N^{*} X$ (see (1.22)) (they will be referred to as normal fields of the first kind);

- vector fields everywhere tangent to the second copy of $N^{*} X$ (they will be referred to as normal fields of the second kind);

- vector fields lifted from $T^{*} X$.

Now we can restate the first part of Definition 1.12 as follows.

Definition 1.13. By $S_{g}^{m, k, l}\left(L_{g}\right)$, where $m, k, l \in \mathbb{R}$, we denote the space of smooth functions $a$ on the manifold $L_{g}$ with the following property: if $\left\{V_{1}, \ldots, V_{j}\right\}$ is an arbitrary (possibly, empty) finite set of vector fields on $L_{g}$ such that each of the fields is homogeneous of degree 0 or 1 and either is normal of the first or second kind or is lifted from $T^{*} X$, then the estimate

$$
\left|V_{1} \cdots V_{j} a(\alpha)\right| \leq C\left(1+\left|\pi_{1}(\alpha)\right|\right)^{m-j_{1}}(1+|p(\alpha)|)^{k-j_{2}}\left(1+\left|\pi_{2}(\alpha)\right|\right)^{l-j_{3}}
$$

holds, where $j_{1}$ is the number of first-order homogeneous normal fields of the first kind, $j_{3}$ is the number of first-order homogeneous normal fields of the second kind, $j_{2}$ is the number of first-order homogeneous normal fields lifted from $T^{*} X, \pi_{1}$ and $\pi_{2}$ are the projections on the first and second factors, respectively, in the Cartesian product $T^{*} M \times T^{*} M$, and $p$ is the projection (1.20).

Thus, we have defined quite a few symbol spaces. To simplify the notation, in the following we usually omit the manifold argument in the notation of symbol spaces, i.e., write, say, $S_{g}^{m, k, l}$ instead of $S_{g}^{m, k, l}\left(L_{g}\right)$, etc., provided this does not lead to a confusion. 


\subsection{The product of symbols (twisted multiplication)}

Let $L_{1}$ and $L_{2}$ be two Lagrangian manifolds (1.25) such that the composition $L=L_{1} \circ L_{2}$ is well defined. Then there is an operation, called the twisted multiplication in what follows, that takes pairs of symbols on $L_{1}$ and $L_{2}$ to symbols on $L$. (See Appendix, Subsection A.7.) This operation involves integration over the fibers of the bundle (1.26). In our situation, the fibers need not be compact, and so the twisted product is defined only under certain restrictions on the types of factors. First, let us write out an expression for the twisted product specifying the general assertion in Appendix.

\subsubsection{The general twisted product formula}

Let the manifold $L_{1}, L_{2}$, and $L$ be equipped with smooth real measures $\mu_{1}, \mu_{2}$, and $\mu$, respectively. We make some choice of the half-densities $\sqrt{\mu_{1}}, \sqrt{\mu_{2}}, \sqrt{\mu}$. For example, we can adopt the convention that $\sqrt{\mu}$ is represented in local coordinates on $L$ by the arithmetic square root $\sqrt{\mid \widetilde{\mu \mid}}$, where $\widetilde{\mu}$ is the density of $\mu$ in these coordinates. Then the twisted multiplication

$$
\begin{aligned}
*: C_{0}^{\infty}\left(L_{1}\right) \times C_{0}^{\infty}\left(L_{2}\right) & \rightarrow C_{0}^{\infty}(L), \\
\left(f_{1}, f_{2}\right) & \mapsto f_{1} * f_{2}
\end{aligned}
$$

can be described as follows. The expression

$$
\begin{gathered}
\left(f_{1} \sqrt{\mu_{1}}\right)(\alpha, \beta)\left(f_{2} \sqrt{\mu_{2}}\right)(\beta, \gamma), \\
(\alpha, \beta) \in L_{1} \subset T^{*} M_{1} \times T^{*} M_{2}, \quad(\beta, \gamma) \in L_{2} \subset T^{*} M_{2} \times T^{*} M_{3},
\end{gathered}
$$

is a half-density on $L$ whose values are densities on the fibers $F_{(\alpha, \gamma)}$ of the bundle (1.26). Hence the expression

$$
\left(f_{1} * f_{2}\right)(\alpha, \gamma)=\frac{1}{\sqrt{\mu}}\left(\frac{1}{2 \pi}\right)^{e} \int_{F_{(\alpha, \gamma)}}\left(f_{1} \sqrt{\mu_{1}}\right)(\alpha, p)\left(f_{2} \sqrt{\mu_{2}}\right)(\beta, \gamma)
$$

is a well-defined function on $L$ (see [Hör83b]). In local coordinates, this function is represented by an ordinary integral over the fiber variables. The normalizing factor $(1 / 2 \pi)^{e}$ is introduced so as to ensure that there is no additional factor in the formulas for products of operators.

If the fiber $F_{(\alpha, \gamma)}$ is compact, then the twisted product (1.38) is defined not only for compactly supported, but also for arbitrary smooth functions. In the general case, we say that the twisted product (1.38) is defined for given functions $f_{1} \in C^{\infty}\left(L_{1}\right)$ and $f_{2} \in C^{\infty}\left(L_{2}\right)$ if the integral on the right-hand side in (1.38) converges absolutely. 


\subsubsection{A twisted product theorem}

In this item, we describe the twisted products (1.37) in the symbol spaces introduced above for the cases corresponding to the compositions of Lagrangian manifolds described in Theorem 1.3 (see Table (1.27)).

First, we consider the case of "trivial" products, where at least one of the factors is the diagonal manifold $L_{M}$ or $L_{X}$. By Theorem 1.3, the fiber of the bundle (1.26) is a singleton, so that the twisted products are always defined. The type of the product can readily be expressed via the types of the factors. Leaving aside the case in which both factors are diagonal (and hence one deals with products of pseudodifferential operators), we state the following theorem.

Theorem 1.14. The twisted product is well defined in the following symbol spaces for any values of the indices:

$$
\begin{aligned}
S^{m}\left(L_{X}\right) * S_{b}^{k, l} & \subset S_{b}^{m+k, l}, \\
S^{m}\left(L_{M}\right) * S_{c}^{m^{\prime}, k} & \subset S_{c}^{m+m^{\prime}, k}, \quad S^{m}\left(L_{M}\right) * S_{g}^{m^{\prime}, k, l} \subset S_{g}^{m+m^{\prime}, k, l}, \\
S_{b}^{k, l} * S^{m}\left(L_{M}\right) & \subset S_{b}^{k, l+m}, \quad S_{g}^{m^{\prime}, k, l} * S^{m}\left(L_{M}\right) \subset S_{g}^{m^{\prime}, k, l+m}, \\
S_{c}^{m^{\prime}, k} * S^{m}\left(L_{X}\right) & \subset S_{c}^{m^{\prime}, k+m} .
\end{aligned}
$$

For any of these compositions, one has

$$
\operatorname{ord}\left(a_{1} * a_{2}\right)=\operatorname{ord} a_{1}+\operatorname{ord} a_{2} .
$$

The following theorem describes the twisted products (1.37) corresponding to the compositions in the table (1.27) for the case in which neither of the factors is $L_{X}$ or $L_{M}$.

Theorem 1.15. The twisted product of symbols corresponding to the compositions of Lagrangian manifolds in the last three rows and columns of Table (1.27) is described by the following table:

\begin{tabular}{c|c|c|c|} 
& $S_{b}^{k_{2}, l_{2}}$ & $S_{c}^{m_{2}, k_{2}}$ & $S_{g}^{m_{2}, k_{2}, l_{2}}$ \\
\hline$S_{b}^{k_{1}, l_{1}}$ & - & $S_{X}^{k_{1}+l_{1}+k_{2}+m_{2}+\nu}$ & $S_{b}^{k_{1}+l_{1}+k_{2}+m_{2}+\nu, l_{2}}$ \\
\hline$S_{c}^{m_{1}, k_{1}}$ & $S_{g}^{m_{1}, k_{1}+k_{2}, l_{2}}$ & - & \\
\hline$S_{g}^{m_{1}, k_{1}, l_{1}}$ & - & $S_{c}^{m_{1}, k_{1}+l_{1}+k_{2}+m_{2}+\nu}$ & $S_{g}^{m_{1}, k_{1}+l_{1}+k_{2}+m_{2}+\nu, l_{2}}$ \\
\hline
\end{tabular}

The twisted product

$$
S_{c}^{m_{1}, k_{1}}\left(L_{c}\right) * S_{b}^{k_{2}, l_{2}}\left(L_{b}\right)
$$

is defined for arbitrary values of the indices, and the remaining four products in Table (1.44) are defined under the condition

$$
l_{1}+m_{2}<-\nu \text {. }
$$

Finally, condition (1.43) holds for each of these products. 
Let us now state the assertion concerning the twisted product of homogeneous principal symbols.

Theorem 1.16. Let $a_{1}$ and $a_{2}$ be classical symbols whose twisted product $a=a_{1} * a_{2}$ is well defined by one of the two preceding theorems. Next, let $a_{10}, a_{20}$, and $a_{0}$ be the homogeneous principal symbols corresponding to $a_{1}, a_{2}$, and a, respectively. Then the twisted product $a_{10} * a_{20}$ is well defined and $a_{10} * a_{20}=a_{0}$.

Coordinate description. Let us give the expressions for $a=a_{1} * a_{2}$ in special coordinate systems for the cases shown in Table (1.44). (The cases described in Theorem 1.14 are left to the reader.) They have the following form:

$$
\begin{aligned}
L_{b} \circ L_{c}: & a\left(x, p^{\prime}\right) & =\left(\frac{1}{2 \pi}\right)^{\nu} \int a_{1}\left(x, p^{\prime}, \tau\right) a_{2}\left(x, p^{\prime}, \tau\right) d \tau ; \\
L_{c} \circ L_{b}: & a\left(x, \tau, p^{\prime}, \tau^{\prime}\right) & =a_{1}\left(x, \tau, p^{\prime}\right) a_{2}\left(x, p^{\prime}, \tau^{\prime}\right) ; \\
L_{g} \circ L_{g}: & a\left(x, \tau, p^{\prime}, \tau^{\prime}\right) & =\left(\frac{1}{2 \pi}\right)^{\nu} \int a_{1}\left(x, \tau, p^{\prime}, \tau^{\prime \prime}\right) a_{2}\left(x, \tau^{\prime \prime}, p^{\prime}, \tau^{\prime}\right) d \tau^{\prime \prime} ; \\
L_{g} \circ L_{c}: & a\left(x, \tau, p^{\prime}\right) & =\left(\frac{1}{2 \pi}\right)^{\nu} \int a_{1}\left(x, \tau, p^{\prime}, \tau^{\prime}\right) a_{2}\left(x, \tau^{\prime}, p^{\prime}\right) d \tau^{\prime} ; \\
L_{b} \circ L_{g}: & a\left(x, p^{\prime}, \tau^{\prime}\right) & =\left(\frac{1}{2 \pi}\right)^{\nu} \int a_{1}\left(x, p^{\prime}, \tau^{\prime \prime}\right) a_{2}\left(x, \tau^{\prime \prime}, p^{\prime}, \tau^{\prime}\right) d \tau^{\prime \prime} .
\end{aligned}
$$

\section{Quantization and the algebra of operator morphisms}

Now we proceed to the second stage in the construction of our operator algebra. Namely, we quantize the classical objects introduced in the previous section. The elements of the algebra are $2 \times 2$ matrices with operator entries. First, we describe these entries, which will be referred to as the main operators, and then deal with the matrices.

\subsection{The main operators}

We have constructed five Lagrangian manifolds with measure in Subsection 1.1 and the corresponding symbol classes in Subsection 1.3. We obtain five types of operators by quantizing these objects, i.e., by considering the corresponding Fourier integral operators.

\subsubsection{Pseudodifferential operators}

The quantization of symbols on $L_{M}$ and $L_{X}$, results in pseudodifferential operators on $M$ and $X$, respectively. This procedure is well known, and we only mention that pseudodifferential operators themselves will be denoted by uppercase letters (for example, 
$D_{M}$ or $D_{X}$ ) and their symbols (or principal symbols, depending on the context) by the corresponding lowercase letters:

$$
\sigma\left(D_{M}\right)=d_{M}, \quad \sigma\left(D_{X}\right)=d_{X}
$$

\subsubsection{Boundary operators}

Definition 2.1. The boundary operator with symbol $a \in S_{b}^{k, l}\left(L_{b}\right)$ is the Fourier integral operators $\widehat{\Phi}_{b}[a]$ on the Lagrangian manifold $L_{b}$ with measure $\mu_{b}$ and symbol $a$.

The boundary operator with principal symbol $a_{0} \in O_{b}^{k, l}\left(L_{b}\right)$ is the operator $\widehat{\Phi}_{b}\left[a_{0}\right] \stackrel{\text { def }}{=}$ $\widehat{\Phi}_{b}[a]$ with symbol $a=\chi\left(\left|p^{\prime}\right|\right) a_{0}$.

Remark 2.2. Owing to the ambiguity in the construction of the canonical operator, the operator $\widehat{\Phi}_{b}[a]$ is determined nonuniquely, modulo operators whose symbols are of lower order than $a$. (The same remark pertains to all other operators that will be constructed in what follows.)

Example 2.3. By taking $a=1$, we obtain the elementary boundary operator

$$
i^{*}: C^{\infty}(M) \longrightarrow C^{\infty}(X)
$$

which takes each function $f \in C^{\infty}(M)$ to the restriction $i^{*} f \in C^{\infty}(X)$ of $f$ to $X$.

Theorem 2.4. Let $a \in S_{b}^{k, l}\left(L_{b}\right)$. Then the operator $\widehat{\Phi}_{b}[a]$ is continuous in the spaces

$$
\Phi_{b}[a]: H^{s}(M) \rightarrow H^{s-1}(X), \quad r=\operatorname{ord} a,
$$

for $s>l+\nu / 2$.

Coordinate description. Let us write out the expression for $\Phi_{b}[a]$ in special coordinates $(x, t)$. The operator $\widehat{\Phi}_{b}[a]$ is given by the formula

$$
\begin{aligned}
\left(\widehat{\Phi}_{b}[a] u\right)(x) & =\left(\frac{1}{2 \pi}\right)^{n+\nu} \int e^{i\left[p^{\prime}\left(x-x^{\prime}\right)-\tau^{\prime} t^{\prime}\right]} a\left(x, p^{\prime}, \tau^{\prime}\right) u\left(x^{\prime}, t^{\prime}\right) d p^{\prime} d \tau^{\prime} d x^{\prime} d t^{\prime} \\
& =\left(\frac{i}{2 \pi}\right)^{\frac{n+\nu}{2}} \int e^{i p^{\prime} x} a\left(x, p^{\prime}, \tau^{\prime}\right) \widetilde{u}\left(p^{\prime}, \tau^{\prime}\right) d p^{\prime} d \tau^{\prime}
\end{aligned}
$$

where $\widetilde{u}\left(p^{\prime}, \tau^{\prime}\right)$ is the Fourier transform of $u\left(x^{\prime}, t^{\prime}\right)$. 


\subsubsection{Coboundary operators}

Definition 2.5. The coboundary operator with symbol $a \in S_{c}^{m, k}\left(L_{c}\right)$ is the Fourier integral operators $\widehat{\Phi}_{c}[a]$ on the Lagrangian manifold $L_{c}$ with measure $\mu_{c}$ and symbol $a$.

The coboundary operator with principal symbol $a_{0} \in O_{c}^{m, k}\left(L_{c}\right)$ is the operator $\widehat{\Phi}_{c}\left[a_{0}\right] \stackrel{\text { def }}{=}$ $\widehat{\Phi}_{c}[a]$ with symbol $a=\chi\left(\left|p^{\prime}\right|\right) a_{0}$.

Example 2.6. By taking $a=1$, we obtain the elementary coboundary operator

$$
i_{*}: \mathcal{D}^{\prime}(X) \longrightarrow \mathcal{D}^{\prime}(M)
$$

which is the $L^{2}$ adjoint of the elementary boundary operator. It can be locally described in the special coordinates $(x, t)$ as follows:

$$
\left[i_{*} g\right](x, t)=g(x) \otimes \delta(t)
$$

where $\delta$ is the Dirac delta function. In other words, $i_{*} g$ is the product of $g$ by the delta function in the transversal directions, or the delta function on $X$ with density $g$, so that one sometimes writes $i_{*} g=g \delta_{X}$.

Theorem 2.7. Let $a \in S_{c}^{m, k}\left(L_{c}\right)$. Then the operator $\widehat{\Phi}_{c}[a]$ is continuous in the spaces

$$
\widehat{\Phi}_{c}[a]: H^{s}(X) \rightarrow H^{s-r}(M), \quad r=\operatorname{ord} a,
$$

for $s<k$.

Remark 2.8. The operator $\widehat{\Phi}_{c}[a]$ is actually continuous in the spaces

$$
\widehat{\Phi}_{c}[a]: H^{s}(X) \rightarrow H^{-m-\nu / 2-\varepsilon}(M)
$$

for $s \geq k$. (Here $\varepsilon>0$ is arbitrary.) This follows from the embedding $H^{s}(X) \subset H^{k-\varepsilon}(X)$ in conjunction with Theorem 2.7. However, we do not consider these values of $s$, since the order of the operator $\widehat{\Phi}_{c}[a]$ is not related to the order of the symbol for these $s$ and composition theorems considered in the next subsection are no longer valid owing to the fact that one cannot neglect lower-order terms in asymptotic expansions of Fourier integrals.

Coordinate description. By analogy with the preceding, we can write out the expression for the coboundary operator in special local coordinates. It has the form

$$
\begin{aligned}
\left(\widehat{\Phi}_{c}[a] u\right)(x, t) & =\left(\frac{1}{2 \pi}\right)^{n+\nu} \int e^{i\left[p^{\prime}\left(x-x^{\prime}\right)+\tau t\right]} a\left(x, \tau, p^{\prime}\right) u\left(x^{\prime}\right) d x^{\prime} d p^{\prime} d \tau \\
& =\left(\frac{1}{2 \pi}\right)^{\nu}\left(\frac{i}{2 \pi}\right)^{n / 2} \int e^{i\left(p^{\prime} x+\tau t\right)} a\left(x, \tau, p^{\prime}\right) \widetilde{u}\left(p^{\prime}\right) d p^{\prime} d \tau .
\end{aligned}
$$




\subsubsection{Green operators}

Definition 2.9. The Green operator with symbol $a \in S_{g}^{m, k, l}\left(L_{g}\right)$ is the Fourier integral operator $\widehat{\Phi}_{g}[a]$ on the Lagrangian manifold $L_{g}$ with measure $\mu_{g}$ and symbol $a$.

The Green operator with principal symbol $a_{0} \in O_{g}^{m, k, l}\left(L_{g}\right)$ is the operator $\widehat{\Phi}_{g}\left[a_{0}\right] \stackrel{\text { def }}{=}$ $\widehat{\Phi}_{g}[a]$ with symbol $a=\chi\left(\left|p^{\prime}\right|\right) a_{0}$.

The following boundedness theorem is valid for Green operators.

Theorem 2.10. Let $a \in S_{g}^{m, k, l}\left(L_{g}\right)$ be a Green symbol. Then the operator $\widehat{\Phi}_{g}[a]$ is continuous in the spaces

$$
\widehat{\Phi}_{g}[a]: H^{s}(M) \rightarrow H^{s-r}(M), \quad r=\text { ord } a,
$$

for $\nu / 2+l<s<\nu / 2+l+k$.

Remark 2.11. It follows from Theorem 2.10 that it makes sense to consider Green symbols only for $k>0$, since otherwise the set of values of $s$ for which the Green operator is continuous in the above-mentioned spaces is empty.

Coordinate description. The expression for a Green operator in special coordinates has the form

$$
\begin{aligned}
\left(\widehat{\Phi}_{g}[a] u\right)(x, t) & =\left(\frac{1}{2 \pi}\right)^{n+2 \nu} \int e^{i\left[p^{\prime}\left(x-x^{\prime}\right)+\tau t-\tau^{\prime} t^{\prime}\right]} a\left(x, \tau, p^{\prime}, \tau^{\prime}\right) u\left(x^{\prime}, t^{\prime}\right) \\
& =\left(\frac{i}{2 \pi}\right)^{\frac{n+\nu}{2}}\left(\frac{1}{2 \pi}\right)^{\nu} \int e^{i\left(p^{\prime} x+\tau t\right)} a\left(x, \tau, p^{\prime}, \tau^{\prime}\right) \widetilde{u}\left(p^{\prime}, \tau^{\prime}\right) d p^{\prime} d \tau^{\prime} d \tau
\end{aligned}
$$

\subsubsection{An interpretation of the main operators as pseudodifferential operators with operator-valued symbols}

In conclusion, we note that, modulo operators with smooth kernel, boundary, coboundary, and Green operators are concentrated on the submanifold $X$. This follows from the form of the corresponding Lagrangian manifolds. Hence the structure of these operators in a neighborhood of $X$ is of main interest. It turns out that in a tubular neighborhood of $X$ these operators can be interpreted as pseudodifferential operators on $X$ with operatorvalued symbols acting in function spaces on the fibers of the normal bundle of $X$. (Recall that a neighborhood of the zero section of the normal bundle is identified via the geodesic exponential map with a tubular neighborhood of $X$.)

By way of example, let us show this for the case of Green operators. 
Indeed, formula (2.7), specifying the operator $\widehat{\Phi}_{g}[a]$ in special coordinates, can be rewritten in the form

$$
\widehat{\Phi}_{g}[a] u(x, t)=\left(\frac{i}{2 \pi}\right)^{n / 2} \int e^{i p x}\left[\frac{i^{\nu / 2}}{(2 \pi)^{3 \nu / 2}} \int e^{i t \tau} a\left(x, p, \tau, \tau^{\prime}\right) \widetilde{u}\left(p, \tau^{\prime}\right) d \tau^{\prime} d \tau\right] d p,
$$

or

$$
\widehat{\Phi}_{g}[a] u(x, t)=H\left(\stackrel{2}{x},-i \frac{1}{d x}\right) u(x, t),
$$

where $H(x, p)$ is the operator-valued symbol acting on functions of the variables $t$ by the formula

$$
H(x, p) v(t)=\left(\frac{i}{2 \pi}\right)^{\nu / 2}\left(\frac{1}{2 \pi}\right)^{\nu} \int e^{i t \tau} a\left(x, p, \tau, \tau^{\prime}\right) \widetilde{v}\left(\tau^{\prime}\right) d \tau^{\prime} d \tau .
$$

Actually, the operator $H(x, p)$ is a Green operator with parameters $(x, p)$ acting in a space of functions of $t$. The symbol of this operator is $a\left(x, p, \tau, \tau^{\prime}\right)$ (where $x$ and $p$ are treated as parameters). The passage

$$
a\left(x, p, \tau, \tau^{\prime}\right) \rightarrow H(x, p) \rightarrow \widehat{\Phi}_{g}[a]
$$

can naturally be interpreted as a two-stage quantization procedure, first along the fibers of the normal bundle of $X$ (which gives an operator-valued symbol over $X$ ) and then along $X$.

\subsection{Composition formulas and adjoint operators}

Composition. Here we state the composition theorem for the main operators, viz. pseudodifferential operators on $M$ and $X$, (co)boundary operators, and Green operators. To unify the statement, we temporarily denote the Fourier integral operator with symbol $a$ on a Lagrangian manifold $L$ from the list $(1.2)$ by $\widehat{\Phi}(L, a)$. (The choice of a measure on $L$ has been described above.)

Theorem 2.12. Let $L_{1}$ and $L_{2}$ be two manifolds from the list (1.2) such that the composition $L_{1} \circ L_{2}$ is well defined, and let $\widehat{\Phi}\left(L_{1}, a_{1}\right)$ and $\widehat{\Phi}\left(L_{2}, a_{2}\right)$ be Fourier integral operators with symbols in some of the classes introduced above such that the composition

$$
\widehat{\Phi}\left(L_{1}, a_{1}\right) \widehat{\Phi}\left(L_{2}, a_{2}\right)
$$

is well defined as a product of bounded operators in accordance with the boundedness theorems. Then the twisted product $a_{1} * a_{2}$ is well defined, and the composition formula

$$
\widehat{\Phi}\left(L_{1}, a_{1}\right) \widehat{\Phi}\left(L_{2}, a_{2}\right) \equiv \widehat{\Phi}\left(L_{1} \circ L_{2}, a_{1} * a_{2}\right)
$$

is valid modulo operators of order $<$ ord $a_{1}+\operatorname{ord} a_{2}$.

The same assertion remains valid if $a_{1}$ and $a_{2}$ are the homogeneous principal symbols of the corresponding operators. 


\section{The adjoint operator.}

Theorem 2.13. Let $L$ be a manifold from the list (1.2), and let $\Phi(L, a)$ be a Fourier integral operator with symbol from some of the classes introduced above such that the operator $\widehat{\Phi}(L, a)$ is well defined as a bounded operator by the boundedness theorems. Then the formally $L^{2}$-adjoint operator is given by the formula

$$
(\widehat{\Phi}(L, a))^{*}=\widehat{\Phi}\left({ }^{T} L, \overline{\left(\varphi^{*}\right)^{-1} a}\right)
$$

modulo operators of order $<$ ord $a_{1}+\operatorname{ord} a_{2}$. Here the bar over the symbol on the right-hand side stands for complex conjugation and

$$
\varphi: L \rightarrow^{T} L
$$

is the mapping obtained by the restriction to $L$ of the transposition of factors in the Cartesian product of cotangent bundles.

The same assertion is valid if by a we mean the homogeneous principal symbol of the Fourier integral operator.

\subsection{Algebras of operator morphisms}

Now we are in a position to construct the operator algebras. They will consist of $2 \times 2$ operator matrices of the form

$$
\left(\begin{array}{cc}
D_{M}+\widehat{\Phi}_{g} & \widehat{\Phi}_{c} \\
\widehat{\Phi}_{b} & D_{X}
\end{array}\right) .
$$

Since the formal "multiplication" of the corresponding matrices of Lagrangian manifolds gives

$$
\left(\begin{array}{cc}
L_{M}+L_{g} & L_{c} \\
L_{b} & L_{X}
\end{array}\right)\left(\begin{array}{cc}
L_{M}+L_{g} & L_{c} \\
L_{b} & L_{X}
\end{array}\right)=\left(\begin{array}{cc}
L_{M}+L_{g} & L_{c} \\
L_{b} & L_{X}
\end{array}\right)
$$

one can hope that the set of operator matrices of this form is indeed closed with respect to composition.

Let us proceed to exact statements.

\subsubsection{Definition of the algebras}

Since the range of the Sobolev smoothness exponent $s$ for which Green, boundary, and coboundary operators are defined is not the entire real line, we see that operators of positive order with such entries will not form an algebra. However, for any $s$ and $\sigma$ one can indicate such symbol spaces for all entries that the operators

$$
\left(\begin{array}{cc}
D_{M}+\widehat{\Phi}_{g} & \widehat{\Phi}_{c} \\
\widehat{\Phi}_{b} & D_{X}
\end{array}\right): \begin{gathered}
H^{s}(M) \\
\bigoplus \\
H^{\sigma}(M)
\end{gathered} \longrightarrow \begin{gathered}
H^{s}(M) \\
\oplus \\
H^{\sigma}(M)
\end{gathered}
$$


will be continuous and form an algebra. We present the corresponding result for $s=\sigma=0$. The general case was analyzed in [SS96].

Let $m_{g}, k_{g}, k_{c}, k_{b} \in \mathbb{R}$ be given numbers satisfying the inequalities

$$
\begin{gathered}
k_{g}>0, k_{c}>0, k_{b}>0, \\
m_{g}<-\frac{\nu}{2}, m_{g}+k_{g}>-\frac{\nu}{2} .
\end{gathered}
$$

(Note that system (2.13) is consistent. For example, one can take $m_{g}=-\nu, k_{g}=\nu$, $k_{c}=k_{b}=1$ as a solution.)

We set

$$
l_{g}=-m_{g}-k_{g}-\nu, m_{c}=-k_{c}-\nu / 2, l_{b}=-k_{b}-\nu / 2 .
$$

The following assertion is a straightforward consequence of the boundedness and composition theorems.

Theorem 2.14. The set $\mathcal{A}\left(m_{g}, k_{g}, k_{c}, k_{b}\right)$ of operator $2 \times 2$ matrices of the form

$$
A=\left(\begin{array}{cc}
D_{M}+\widehat{\Phi}_{g}\left[a_{g}\right] & \widehat{\Phi}_{c}\left[a_{c}\right] \\
\widehat{\Phi}_{b}\left[a_{b}\right] & D_{X}
\end{array}\right),
$$

where $D_{M}$ and $D_{X}$ are zero-order pseudodifferential operators on $M$ and $X$, respectively, and the symbols $a_{g}, a_{c}$, and $a_{b}$ lie in the spaces

$$
a_{g} \in S_{g}^{m_{g}, k_{g}, l_{g}}\left(L_{g}\right), \quad a_{c} \in S_{c}^{m_{c}, k_{c}}\left(L_{c}\right), \quad a_{b} \in S_{b}^{k_{b}, l_{b}}\left(L_{b}\right),
$$

is a linear space of continuous operators in $L^{2}(M) \oplus L^{2}(X)$.

The (nondirect) sum

$$
\mathcal{A}=+\mathcal{A}\left(m_{g}, k_{g}, k_{c}, k_{b}\right)
$$

over all $m_{g}, k_{g}, k_{c}, k_{b}$ satisfying (2.13) is an algebra.

Remark 2.15. From the practical viewpoint, it is of interest to consider operators of nonzero order in Sobolev spaces other than $L^{2}$; theoretically, it suffices to study the algebras described in Theorem 2.14, since an operator of the form (2.15) acting from $H^{s_{1}}(M) \oplus H^{s_{2}}(X)$ into $H^{\sigma_{1}}(M) \oplus H^{\sigma_{2}}(X)$ can always be reduced to an operator in the space $L^{2}(M) \oplus L^{2}(X)$ : one simply multiplies it on the right and on the left by appropriate diagonal matrices of pseudodifferential operators.

Remark 2.16. Let $m_{g}, k_{g} \in \mathbb{R}$ be given numbers satisfying the inequalities

$$
m_{g}<-\nu / 2<m_{g}+k_{g} .
$$

We set

$$
k_{b}=m_{g}+k_{g}+\nu / 2 \quad k_{c}=-m_{g}-\nu / 2 .
$$

Then inequalities (2.13) are satisfied automatically. We write

$$
\mathcal{A}\left(m_{g}, k_{g}\right)=\mathcal{A}\left(m_{g}, k_{g}, k_{c}, k_{b}\right),
$$

where $k_{b}$ and $k_{c}$ are given by formulas (2.17). 
A careful computation of the exponents according to Table (1.44) in the composition theorem 1.15 shows that $A\left(m_{g}, k_{g}\right) \subset A$ is a subalgebra.

In what follows we mainly deal with subalgebras of the algebras defined above, formed by classical operators (i.e., operators with classical symbols).

\subsubsection{Involution}

Theorem 2.13 implies the following assertion.

Theorem 2.17. The algebra $\mathcal{A}$ is an involutive algebra with involution taking each operator to its $L^{2}$ adjoint. Moreover,

$$
\mathcal{A}\left(m_{g}, k_{g}, k_{c}, k_{b}\right)^{*}=\mathcal{A}\left(m_{g}^{*}, k_{g}^{*}, k_{c}^{*}, k_{b}^{*}\right)
$$

where

$$
m_{g}^{*}=-m_{g}-k_{g}-\nu, \quad k_{g}^{*}=k_{g}, \quad k_{c}^{*}=k_{b}, \quad k_{b}^{*}=k_{c} .
$$

In particular, the subalgebras

$$
\mathcal{A}(-(k+\nu) / 2, k) \subset \mathcal{A}
$$

are invariant with respect to involution.

\subsubsection{Green subalgebras}

There is an important subalgebra of $\mathcal{A}$ formed by matrices of the form

$$
\left(\begin{array}{cc}
\widehat{\Phi}_{g} & 0 \\
0 & 0
\end{array}\right)
$$

This subalgebra is naturally isomorphic to the algebra of operators of the form $\widehat{\Phi}_{g}$ in $L^{2}(M)$. (As follows from the composition theorem, such operators indeed form an algebra.) More precisely, let us give the following definition. Let numbers $m, k, l \in \mathbb{R}$ satisfy the relations

$$
\left\{\begin{array}{l}
m+k+l+\nu=0 \\
l+\nu / 2<0<l+\nu / 2+k .
\end{array}\right.
$$

We set $O^{m, l}=O_{g}^{m, k, l}\left(L_{g}\right)$. (Note that the notation is adequate, since

$$
k=k(m, l)=-m-l-\nu
$$

is uniquely determined by $m$ and $l$.) 
Definition 2.18. The Green algebra $\mathcal{A}_{g}(m, l)$ is the set of continuous linear operators in $L^{2}(M)$ representable in the form

$$
A=\widehat{\Phi}_{g}[a]+K
$$

where $a \in O^{m, l}$ and $K$ is a compact operator in $L^{2}(M)(K \in \mathcal{K})$. Elements of the Green algebra are called Green operators. tion).

Green algebras play an important role in the ellipticity conditions (see the next sec-

\section{$3 \quad$ Elliptic morphisms and the index formula}

In this section, we give the definition of ellipticity for morphisms, prove theorems concerning the Fredholm property, and present an index formula for elliptic morphisms that do not contain Green operators. In the ellipticity conditions for general morphisms, the almost inversion of operators of the form $1+T$, where $T=\widehat{\Phi}_{g}[a]$ is a Green operator, plays an important role. We start our exposition with a study of such operators.

\subsection{The Green equation}

\subsubsection{Statement of the problem}

Consider the equation

$$
u+\widehat{\Phi}_{g}[a] u=f \in L^{2}(M)
$$

for an unknown function $u \in L^{2}(M)$, where $a \in O^{(m, l)}$ is a given principal symbol. In more detailed notation, Eq. (3.1) has the form

$$
u(x, t)+\int K\left(x, t, x^{\prime}, t^{\prime}\right) u\left(x^{\prime}, t^{\prime}\right) d x^{\prime} d t^{\prime}=f(x, t),
$$

where the kernel $K\left(x, t, x^{\prime}, t^{\prime}\right)$ is given by the formula

$$
K\left(x, t, x^{\prime}, t^{\prime}\right)=\left(\frac{1}{2 \pi}\right)^{n+2 \nu} \int e^{i\left[p\left(x-x^{\prime}\right)+\tau t-\tau^{\prime} t^{\prime}\right]}(\chi a)\left(x, p, \tau, \tau^{\prime}\right) d p d \tau d \tau^{\prime}
$$

Equation (3.1) (or (3.2)) will be called the Green equation of the second kind, or simply the Green equation. As is often the case in the theory of integral equations, one cannot solve Eq. (3.1) exactly or even write out informative conditions guaranteeing the unique solvability (except for the trivial case in which the norm of the operator $\widehat{\Phi}_{g}[a]$ is less than unity). Thus, we proceed to the analysis of conditions under which Eq. (3.1) is Fredholm. Here one can already obtain nontrivial results, namely, a criterion for the Fredholm property of Eq. (3.1) in terms of the principal symbol $a$. This criterion is described in the subsequent items. 


\subsubsection{An equation for the symbol of an almost inverse}

It is well known that an equation is Fredholm if and only if the operator specifying the equation has a (two-sided) almost inverse. Let us seek a right almost inverse of $1+\widehat{\Phi}_{g}[a]$ in the form $1+\widehat{\Phi}_{g}[b]$, where $b \in O^{(m, l)}$. Then we have

$$
\left(1+\widehat{\Phi}_{g}[a]\right)\left(1+\widehat{\Phi}_{g}[b]\right)=1+\widehat{\Phi}_{g}[a+b+a * b]
$$

modulo compact operators, and for the symbol $b$ we obtain the equation

$$
b+a * b=-a
$$

Likewise, if we seek a left almost inverse in the form $1+\widehat{\Phi}_{g}[c]$, then for $c$ we obtain the equation

$$
c+c * a=-a \text {. }
$$

\subsubsection{Ellipticity and the Fredholm property for the Green equation}

Here we state necessary and sufficient conditions for the Fredholm property of Eq. (3.1). The remaining items deal with the proof of the theorem stated in this item.

Definition 3.1. We say that the Green equation (3.1) (and the operator $1+\widehat{\Phi}_{g}[a]$ ) is elliptic if the equation

$$
w+a * w=v
$$

has a solution $w \in O^{(m, l)}$ for every right-hand side $v \in O^{(m, l)}$.

Remark 3.2. The solvability of Eq. (3.7) with arbitrary right-hand side is equivalent to the solvability of Eq. (3.5) (i.e., Eq. (3.7) with special right-hand side $-a$ ). Indeed, one of the implications is trivial; as to the other, if $b$ is a solution of (3.5), then the solution of (3.7) for arbitrary $v$ is given by the formula

$$
w=b+a+v+b *(a+v),
$$

which can readily be verified by a straightforward substitution into the equation.

Theorem 3.3. The Green equation (3.1) is Fredholm if and only if it is elliptic.

Corollary 3.4. The operator $1+\widehat{\Phi}_{g}[a] \in \mathcal{A}_{g}^{(m, l)}$ is Fredholm if and only if it has an almost inverse (a regularizer) in the algebra $\mathcal{A}_{g}^{(m, l)}$.

Indeed, the symbol of the regularizer is just given by the solution of Eq. (3.5).

Remark 3.5. Note that the ellipticity condition does not mention Eq. (3.6) or the unique solvability of Eq. (3.5). We shall see from the proof that the solvability of Eq. (3.5) is equivalent to the solvability of Eq. (3.6) and is equivalent to the unique solvability of either of these equations. 
Proof. The proof splits into several stages described in the forthcoming items. Let us rewrite Eq. (3.7) in coordinates:

$$
w\left(x, p, \tau, \tau^{\prime}\right)+\left(\frac{1}{2 \pi}\right)^{\nu} \int_{\mathbb{R}^{\nu}} a\left(x, p, \tau, \tau^{\prime \prime}\right) w\left(x, p, \tau^{\prime \prime}, \tau^{\prime}\right) d \tau^{\prime \prime}=v\left(x, p, \tau, \tau^{\prime}\right) .
$$

It is natural to treat this equation as a family of equations depending on the parameters $\left(x, p, \tau^{\prime}\right)$. Let us study the properties of this equation. First, without loss of generality we can assume that $m=l$. Indeed, we set $\widetilde{m}=\frac{1}{2}(m, l)$ and $\varkappa=\frac{1}{4}(m-l)$. The map

$$
a\left(x, p, \tau, \tau^{\prime}\right) \longmapsto\left(p^{2}+\tau^{2}\right)^{-\varkappa} a\left(x, p, \tau, \tau^{\prime}\right)\left(p^{2}+\tau^{\prime 2}\right)^{\varkappa}
$$

is an isomorphism of $O^{(m, l)}$ onto $O^{\widetilde{m}, \widetilde{m}}$; the form of Eq. (3.9) remains unchanged if we apply the transformation (3.10) to all functions $w, a, v$ occurring in the equation. Thus, from now on we assume that $m=l$. Then it follows from the restrictions imposed on $m$, $l$, and $k$ that

$$
m<-\nu / 2 \text {. }
$$

Since

$$
\left|a\left(x, p, \tau, \tau^{\prime}\right)\right| \leq C|p|^{k}(|p|+|\tau|)^{m}\left(|p|+\left|\tau^{\prime}\right|\right)^{m},
$$

we see that the kernel in (3.9) is square integrable with respect to the variables $\tau, \tau^{\prime \prime}$, so that for any given $\left(x, p, \tau^{\prime}\right), p \neq 0$, this equation is a Fredholm integral equation of the second kind in the space $L^{2}\left(\mathbb{R}_{\tau}^{\nu}\right)$. It follows from Fredholm theory that this equation is solvable in $L^{2}\left(\mathbb{R}_{\tau}^{\nu}\right)$ for any right-hand side if and only if the homogeneous equation has no nonzero solutions in $L^{2}\left(\mathbb{R}_{\tau}^{\nu}\right)$. However, we are interested in solutions of this equation in $O^{(m, l)}$. First, consider the case in which the homogeneous equations (3.9) have no nonzero solutions in $L^{2}\left(\mathbb{R}_{\tau}^{\nu}\right)$ for any $(x, p), p \neq 0$.

Proposition 3.6. In the absence of nonzero solutions of the homogeneous equation, the solution in $L^{2}\left(\mathbb{R}_{\tau}^{\nu}\right)$ of $E q$. (3.9) with right-hand side in $O^{(m, m)}$ lies in $O^{m, m}$.

Proof. The desired $\mathbb{R}_{+}$-homogeneity of the solution follows from the $\mathbb{R}_{+}$-homogeneity of the kernel and the right-hand side in conjunction with the uniqueness of the solution. Next, the function $w$ is obtained from $v$ by an application of the continuous inverse in $L^{2}\left(\mathbb{R}_{\tau}^{\nu}\right)$ to the Fredholm operator of the second kind in Eq. (3.9), so that the differentiability of $w$ (viewed as a function ranging in $L^{2}$ ) with respect to variables $(\tau, x, p)$ is obvious. Now it is easy to prove the desired estimates for $w$ and the derivatives of $w$ by induction over the order of the derivative, using the relation obtained from (3.9) by the transposition of the integral term to the right-hand side, which completes the proof.

Note that Eq. (3.6) is the complex conjugate of the adjoint equation of (3.5), and it follows from Fredholm theory that both equations are solvable or unsolvable simultaneously.

The case in which the homogeneous Fredholm equation has a nontrivial solution is consider in the next item. 
3.1.4 The operator $1+\widehat{\Phi}_{g}[a]$ is not Fredholm if Eq. (3.9) is not uniquely solvable Proposition 3.7. Suppose that the homogeneous equation

$$
\varphi(\tau)+\left(\frac{1}{2 \pi}\right)^{\nu} \int_{\mathbb{R}^{\nu}} a\left(x, p, \tau, \tau^{\prime \prime}\right) \varphi\left(\tau^{\prime \prime}\right) d \tau^{\prime \prime}=0
$$

has a solution $\varphi \in L^{2}\left(\mathbb{R}^{\nu}\right)$ for some values $(x, p)=\left(x_{0}, p_{0}\right) \in \mathbb{R}^{n} \times \mathbb{R}^{n} \backslash\{0\}$. Then the operator $1+\widehat{\Phi}_{g}[a]$ is not Fredholm.

Proof. Without loss of generality, we can assume that $\left|p_{0}\right|=1$. We shall construct a family of functions $u_{\lambda} \in L^{2}(M), \lambda \in \mathbb{R}_{+}$, with the following properties:

(a) $\left\|u_{\lambda}\right\|=1$ for all $\lambda$;

(b) $\left\|\left(1+\widehat{\Phi}_{g}[a]\right) u_{\lambda}\right\| \rightarrow 0$ for $\lambda \rightarrow \infty$;

(c) $u_{\lambda}$ weakly converges to 0 as $\lambda \rightarrow \infty$.

The existence of such a sequence implies that $1+\widehat{\Phi}_{g}[a]$ is not Fredholm. Indeed, we argue by contradiction: let $1+\widehat{\Phi}_{g}[a]$ be Fredholm, and let $R$ be a regularizer, so that

$$
R\left(1+\widehat{\Phi}_{g}[a]\right)=1+K, \quad K \in \mathcal{K} .
$$

Then

$$
u_{\lambda}=R\left(1+\widehat{\Phi}_{g}[a]\right) u_{\lambda}-K u_{\lambda} \rightarrow 0
$$

as $\lambda \rightarrow \infty$ by (b),(c), and the fact that a compact operator takes each weakly convergent sequence to a strongly convergent sequence. But Eq. (3.12) contradicts (a).

We set

$$
u_{\lambda}(x, t)=c_{\lambda} e^{i \lambda\left(x-x_{0}\right) p_{0}} \psi\left(\sqrt{\lambda}\left(x-x_{0}\right)\right) \breve{\varphi}(\lambda t),
$$

where $c_{\lambda}$ is a constant normalizing $u_{\lambda}$ in $L^{2}, u(x)$ is a smooth compactly supported function, and $\breve{\varphi}(t)$ is the inverse Fourier transform of $\varphi(\tau)$. The function (3.13) satisfies condition (a) by construction. Next, one can readily see that this function weakly converges to zero as $\lambda \rightarrow \infty$ (i.e., condition (c) holds): it suffices to show that

$$
\int u_{\lambda}(x, t) \varphi_{1}(x) \varphi_{2}(t) d x d t \rightarrow 0
$$

as $\lambda \rightarrow \infty$ for any smooth compactly supported functions $\varphi_{1}$ and $\varphi_{2}$, but this can readily be proved by integration by parts with respect to $x$ with regard to the fact that $c_{\lambda} \sim$ 
$\lambda^{n / 2+\nu}$. It remains to verify condition (b). Using the representation (2.8)-(2.9) of the Green operator as a pseudodifferential operator with operator-valued symbol, we obtain

$$
\begin{aligned}
\left(1+\widehat{\Phi}_{g}[a]\right) u_{\lambda} & \equiv\left(1+H\left(x_{1},-i \frac{\partial}{\partial x}\right)\right) u_{\lambda} \\
& =c_{\lambda} e^{i \lambda\left(x-x_{0}\right) p_{0}} \psi\left(\sqrt{\lambda}\left(x-x_{0}\right)\right)\left(1+H\left(x, \lambda p_{0}\right)\right) \breve{\varphi}(\lambda t)+o(1) \\
& =c_{\lambda} e^{i \lambda\left(x-x_{0}\right) p_{0}} \psi\left(\sqrt{\lambda}\left(x-x_{0}\right)\right)\left(1+H\left(x, \lambda p_{0}\right)\right) \breve{\varphi}(\lambda t)+o(1) .
\end{aligned}
$$

But

$$
\begin{aligned}
\left(1+H_{0}\left(x_{0}, \lambda\right.\right. & \left.\left.p_{0}\right)\right) \breve{\varphi}(\lambda t) \\
& =\lambda^{-\nu} \mathcal{F}_{\tau \rightarrow t}^{-1}\left\{\varphi\left(\frac{\tau}{\lambda}\right)+\left(\frac{1}{2 \pi}\right)^{\nu} \int_{\mathbb{R}^{\nu}} a\left(x_{0}, \lambda p_{0}, \tau, \tau^{\prime \prime}\right) \varphi\left(\frac{\tau^{\prime \prime}}{\lambda}\right) d \tau^{\prime \prime}\right\} \\
& =\lambda^{-\nu} \mathcal{F}_{\tau \rightarrow t}^{-1}\left\{\varphi\left(\frac{\tau}{\lambda}\right)+\left(\frac{1}{2 \pi}\right)^{\nu} \int_{\mathbb{R}^{\nu}} a\left(x_{0}, p_{0}, \frac{\tau}{\lambda}, \frac{\tau^{\prime \prime}}{\lambda}\right) \varphi\left(\frac{\tau^{\prime \prime}}{\lambda}\right) d\left(\frac{\tau^{\prime \prime}}{\lambda}\right)\right\}=0,
\end{aligned}
$$

whence it follows that (b) is also satisfied.

\subsubsection{Completion of the proof}

Now the assertion of the theorem becomes obvious: if the symbol equation is not uniquely solvable at least at one point $(x, p), p \neq 0$, then the operator $1+\widehat{\Phi}_{g}[a]$ is not Fredholm, which shows that the ellipticity condition is necessary. On the other hand, if the ellipticity condition is satisfied, then one can construct left and right regularizers by solving the corresponding symbol equations, thus proving the Fredholm property. The proof is complete.

\subsection{Ellipticity and the Fredholm property}

In this subsection, we establish necessary and sufficient conditions for a given morphism

$$
A=\left(\begin{array}{cc}
D_{M}+\widehat{\Phi}_{g}[g] & \widehat{\Phi}_{c}[c] \\
\widehat{\Phi}_{b}[b] & D_{X}
\end{array}\right) \in \mathcal{A}
$$

to have an almost inverse (an inverse modulo compact operators) $A^{-1} \in \mathcal{A}$ in the algebra $\mathcal{A}$. These conditions are called ellipticity conditions. First, we consider ellipticity conditions for morphisms (3.14) with $\widehat{\Phi}_{g}[g]=0$. They are of main interest for two reasons:

- practically interesting problems (Sobolev problems) result only in morphisms that do not contain Green operators; 
- in this case, one can obtain ellipticity conditions (and an index formula) in a finite (algebraic) form.

Thus, we consider an operator (3.14) without the Green term, i.e., an operator of the form

$$
A=\left(\begin{array}{cc}
D_{M} & \widehat{\Phi}_{c}[c] \\
\widehat{\Phi}_{b}[b] & D_{X}
\end{array}\right)
$$

We seek a (right) almost inverse in the form

$$
A^{-1}=\left(\begin{array}{cc}
\widetilde{D}_{M}+\widehat{\Phi}_{g}[\varphi] & \widehat{\Phi}_{c}[\widetilde{c}] \\
\widehat{\Phi}_{b}[\widetilde{b}] & \widetilde{D}_{X}
\end{array}\right)
$$

and, multiplying by $A$ on the left, arrive at the system of equations

$$
\left(\begin{array}{cc}
D_{M} & \widehat{\Phi}_{c}[c] \\
\widehat{\Phi}_{b}[b] & P_{X}
\end{array}\right)\left(\begin{array}{cc}
\widetilde{D}_{M}+\widehat{\Phi}_{g}[\varphi] & \widehat{\Phi}_{c}[\widetilde{c}] \\
\widehat{\Phi}_{b}[\tilde{b}] & \widetilde{D}_{X}
\end{array}\right)=\left(\begin{array}{ll}
1 & 0 \\
0 & 1
\end{array}\right)
$$

which must be satisfied modulo compact operators. Let us compute the operator on the left-hand side in (3.17). Modulo compact operators, it has the form

$$
\left(\begin{array}{cc}
\widetilde{\widetilde{D}}_{M}+\widehat{\Phi}_{g}[\psi] & \widehat{\Phi}_{c}[\widetilde{\widetilde{c}}] \\
\widehat{\Phi}_{b}[\widetilde{\widetilde{b}} & \widetilde{\widetilde{D}}_{X}
\end{array}\right)
$$

where

$$
\widetilde{\widetilde{d}}_{M}=d_{M} \widetilde{d}_{M}, \quad \psi=c * \widetilde{b}+d_{M} * \varphi
$$

and the formulas for $\widetilde{c}, \widetilde{\widetilde{b}}$, and $\widetilde{\widetilde{d}}_{x}$ can readily be written out with the use of the composition law in the algebra $A$. In $(3.18), d_{M}, \widetilde{\widetilde{d}}_{M}$, and $\widetilde{\widetilde{d}}_{M}$ are the principal symbols of the pseudodifferential operators $D_{M}, \widetilde{D}_{M}$, and $\widetilde{\widetilde{D}}_{M}$, respectively. It follows from (3.17) and (3.18) that

$$
d_{M} \widetilde{d}_{M}=1
$$

Thus, a necessary condition for the almost invertibility of the element (3.15) in the algebra $\mathcal{A}$ is the ellipticity of the pseudodifferential operator $D_{M}$. Assuming that this condition is satisfied, we make some transformations of $A$ that do not affect the almost invertibility.

Namely, we subtract the first row multiplied on the left by $\widehat{\Phi}_{b}[b] D_{M}^{-1}$ from the second row. (Here $D_{M}^{-1}$ is an almost inverse of $D_{M}$; we freely use this type of notation, which is unlikely to result in a confusion.) As a result, modulo compact operators we obtain the upper-triangular operator matrix

$$
A^{\prime}=\left(\begin{array}{cc}
D_{M} & \widehat{\Phi}_{c}[c] \\
0 & D_{X}-\widehat{\Phi}_{b}[b] D_{M}^{-1} \widehat{\Phi}_{c}[c]
\end{array}\right)
$$


An upper-triangular matrix is almost invertible if and only if so are its diagonal entries. Thus, we arrive at the following definition.

Definition 3.8. An element (3.15) of the algebra $A$ is said to be elliptic if the following conditions are satisfied:

(i) $D_{M}$ is an elliptic operator on $M$;

(ii) the operator

$$
\Delta_{A}=D_{X}-\widehat{\Phi}_{b}[b] D_{M}^{-1} \widehat{\Phi}_{c}[c]
$$

(the noncommutative determinant) is an elliptic operator on $X$.

Remark 3.9. The second condition possibly deserves an explanation. By the composition theorem, the product $\widehat{\Phi}_{b}[b] D_{M}^{-1} \widehat{\Phi}_{c}[c]$ is a pseudodifferential operator on $X$. Hence so is the noncommutative determinant $\Delta_{A}$, and we can state the ellipticity condition in finite (algebraic) form at least if the symbols occurring in $\Delta_{A}$ are classical. The symbol of $\Delta_{A}$ has the form

$$
\sigma\left(\Delta_{A}\right)=d_{X}-b * d_{M}^{-1} * c,
$$

where $b$ and $c$ are the symbols of $\widehat{\Phi}_{b}$ and $\widehat{\Phi}_{c}$, respectively, and the twisted product $*$ in the general form is given by (1.37) and (1.38) and is described in specific cases in the composition theorem 1.15 and the subsequent text. In special local coordinates, the symbol (3.21) can be represented in the form

$$
\sigma\left(\Delta_{A}\right)(x, p)=d_{x}(x, p)+\left(\frac{1}{2 \pi}\right)^{\nu} \int b(x, p, \tau) c(x, p, \tau) d_{M}^{-1}(x, 0, p, \tau) d \tau .
$$

If the symbols $b(x, p, \tau)$ and $c(x, p, \tau)$ (as well as $d_{x}(x, p)$ and $d_{M}(x, t, p, \tau)$ ) are classical, i.e., have homogeneous leading components, then so is the symbol $\sigma\left(\Delta_{A}\right)$, and the ellipticity condition acquires a finite form.

The preceding reasoning implies the following theorem.

Theorem 3.10. Let $A$ be an elliptic morphism of the form (3.15). Then $A$ is Fredholm.

Let us now state the ellipticity conditions for general morphisms (3.14) containing a nonzero Green operator in the upper left corner. We seek an almost inverse in the form (3.16); then the first relation in (3.18) remains the same. Thus, the ellipticity of $D_{M}$ is still necessary for the almost invertibility of $A$ in the algebra. However, the upper left corner of $A$ is now

$$
A_{11}=D_{M}+\widehat{\Phi}_{g}[g],
$$

and the almost invertibility of $A_{11}$ is not necessary for the almost invertibility of $A$ in the algebra, so that the reduction to the upper-triangular case simply does not work. 
Remark 3.11. The special case in which the morphism (3.22) is still almost invertible (one can study this property using the representation

$$
A_{11}=D_{M}\left(1+D_{M}^{-1} \widehat{\Phi}_{g}[g]\right)=D_{M}\left(1+\widehat{\Phi}_{g}\left[d_{M}^{-1} * g\right]\right)
$$

and then analyzing the Green equation with the operator $1+\widehat{\Phi}_{g}\left[d_{M}^{-1} * g\right]$ ) was considered in [SS96]. In this case, the reduction to a triangular form can be carried out with $D_{M}$ replaced by $D_{M}+\widehat{\Phi}_{g}[g]$. In particular, the noncommutative determinant acquires the form

$$
\Delta_{A}=D_{X}-\widehat{\Phi}_{b}[b]\left(D_{M}+\widehat{\Phi}_{g}[g]\right)^{-1} \widehat{\Phi}_{c}[c],
$$

and sufficient ellipticity conditions are that the operators (3.22) and (3.23) must be elliptic. By the results of the preceding subsection concerning the Green equation, the operator $\left(D+\widehat{\Phi}_{g}[g]\right)^{-1}$ again has the form of a pseudodifferential operator plus a Green operator, so that the noncommutative determinant (3.23) proves to be a pseudodifferential operator on $X$ by the composition theorems.

We consider the general case without the assumption that the operator (3.22) is almost invertible. How to state the ellipticity conditions then? To this end, note that the almost invertibility of $A$ in the algebra is equivalent to the simultaneous almost invertibility of $A^{*} A$ and $A A^{*}$. Now we note that the almost invertibility of $A^{*} A$ implies the almost invertibility of $\left(A^{*} A\right)_{11}$. (The same is also true of $A A^{*}$.) This is a straightforward consequence of the following proposition.

Proposition 3.12. If

$$
C=\left(\begin{array}{ll}
a & b \\
c & d
\end{array}\right)
$$

is a nonnegative self-adjoint operator matrix $\left(C=C^{*} \geq 0\right)$ and $C$ is almost invertible, then a is almost invertible.

Proof. The proof is based on the following auxiliary lemma:

Lemma 3.13. For a bounded operator $B=B^{*} \geq 0$, the following assertions are equivalent:

1. B is not Fredholm;

2. there exists a sequence of vectors $u_{j}$ such that $\left\|u_{j}\right\|=1, u_{j} \stackrel{\text { weakly }}{\longrightarrow} 0$, and $\left(u_{j}, B u_{j}\right) \longrightarrow 0$.

Indeed, $\left(u_{j}, B u_{j}\right)=\left\|\sqrt{B} u_{j}\right\|$, whence we see that the condition $\left(u_{j}, B u_{j}\right) \rightarrow 0$ is equivalent to $B u_{j} \rightarrow 0$. Now the implication $(2) \Rightarrow(1)$ is obvious, and to prove $(1) \Rightarrow(2)$ one can take an orthonormal sequence $u_{j} \in R\left(f_{j}(B)\right)$, where

$$
f_{j}(x)= \begin{cases}1, & x \leq 1 / j \\ 0, & x>1 / j\end{cases}
$$


Such a sequence always exists, since for non-Fredholm $B$ all projections $f_{j}(B)$ are of infinite rank.

Now it is easy to prove Proposition 3.12. First, it is clear that $a$ is self-adjoint and nonnegative. If $a$ is not Fredholm, we find a sequence $u_{j}$ with the properties stated in the lemma (for $B=a$ ), and it remains to note that the sequence

$$
v_{j}{ }^{t}\left(u_{j}, 0\right)
$$

can be used to prove that $C$ is not Fredholm, since

$$
\left(v_{j}, C v_{j}\right)=\left(u_{j}, a u_{j}\right) .
$$

Thus, the operators $A^{*} A$ and $A A^{*}$ already fall within the framework of the special case considered in [SS96], so that we can state the ellipticity conditions for $A$ in the following form.

Definition 3.14. The morphism (3.14) is said to be elliptic if

1. the operator $D_{M}$ is elliptic;

2. the operators $\left(A^{*} A\right)_{11}$ and $\left(A A^{*}\right)_{11}$ are elliptic;

3. the noncommutative determinants $\Delta_{A^{*} A}$ and $\Delta_{A A^{*}}$ are elliptic.

The above reasoning implies the following theorem.

Theorem 3.15. If the morphism (3.14) is elliptic, then it is Fredholm.

Remark 3.16. We have stated the theorem on the Fredholm property for elements of the algebra $A$, i.e., morphisms of order zero acting from the space $L^{2}(X) \oplus L^{2}(M)$ into itself. Since the multiplication by pseudodifferential operators does not change the structure of the operators in question, the ellipticity conditions and the Fredholm property theorem for operators of nonzero order have the same form. This remark pertains to the index theorem (see the next subsection) as well.

Remark 3.17. One can prove that the ellipticity conditions in Definition 3.14 are actually redundant and it suffices to require the ellipticity of $D_{M}$ and the operators in (2) and (3) for only one of the two operators $A^{*} A$ and $A A^{*}$. The situation resembles that for Green equations, where it suffices to require only the solvability of the equation for the symbol of one of the regularizers, right or left. 


\subsection{The index theorem for elliptic morphisms}

Here we state a theorem on the index of morphisms elliptic in the sense of Definition 3.8.

Theorem 3.18. Let

$$
A=\left(\begin{array}{cc}
D_{M} & \widehat{\Phi}_{c} \\
\widehat{\Phi}_{b} & D_{X}
\end{array}\right)
$$

be an elliptic element of the algebra $\mathcal{A}$ in the sense of Definition 3.8. Then the index of the operator $A$ is given by the formula

$$
\text { ind } A=\text { ind } D_{M}+\text { ind } \Delta_{A},
$$

where $\Delta_{A}$ is the noncommutative determinant (3.20).

Proof. One has

$$
\left(\begin{array}{cc}
1 & 0 \\
-\widehat{\Phi}_{b} D_{M}^{-1} & 1
\end{array}\right) A=\left(\begin{array}{cc}
D_{M} & \widehat{\Phi}_{c} \\
0 & \Delta_{A}
\end{array}\right)
$$

modulo compact operators. (This identity has actually been used in the proof of the ellipticity conditions). Since the index of a product of operators is equal to the sum of indices of the factors and the index of a triangular matrix is equal to the sum of indices of its diagonal entries, we arrive at the desired formula (3.24). The proof is complete.

\section{The Sobolev problem}

Now we apply the results to Sobolev problems. First, we describe general Sobolev problems and clarify their relation to elliptic morphisms.

\subsection{Sobolev problems and elliptic morphisms}

Let $X \stackrel{i}{\hookrightarrow} M$ be an embedding of smooth compact manifolds with $\operatorname{codim} X=\nu$,

$$
D: H^{s}\left(M, E_{1}\right) \rightarrow H^{s-m}\left(M, E_{2}\right)
$$

an elliptic pseudodifferential operators of order $m$ acting in spaces of sections of bundles $E_{1}$ and $E_{2}$ over $M$, and

$$
B: H^{s}\left(M, E_{1}\right) \rightarrow H^{s-b}(M, F)
$$

a pseudodifferential operator of order $b$ acting in spaces of sections of the bundles $E_{1}$ and $F$ over $M$. To these data, we assign the problem

$$
\left\{\begin{array}{l}
D u \equiv f \quad \bmod H^{s-m}\left(M, X, E_{2}\right) \\
i^{*} B u=g \in H^{s-b-\nu / 2}\left(X,\left.F\right|_{X}\right)
\end{array}\right.
$$


for an unknown function $u \in H^{s}(M, E)$. This problem will be called the Sobolev problem. We assume that $s>b+\nu / 2$, so that the restriction $i^{*} B u$ is well defined. In (4.1), by $H^{s-m}\left(M, X, E_{2}\right)$ we denote the subspace of $H^{s-m}\left(X, E_{2}\right)$ formed by distributions supported on $X$.

It was shown in [Ste76] (see also [SS96]) that problem (4.1) is equivalent to the system

$$
\left\{\begin{array}{l}
D u+i_{l *} v=f \\
i^{*} B u=g
\end{array}\right.
$$

for the unknown functions $u \in H^{s}(M, E)$ and

$$
v \in \widetilde{H}^{s-m+\nu / 2}\left(X, \mathcal{J}_{x}^{l}\left(E_{2}\right)\right) .
$$

Here $\mathcal{J}_{x}^{l}\left(E_{2}\right)$ is the bundle of $l$ th-order normal jets of the bundle $E_{2}$ on $X$; the elements (4.3) can be represented in an arbitrary special coordinate system $(x, t)$ in the form

$$
v=\left\{v_{\alpha}\right\}_{|\alpha| \leq l}, \quad v_{\alpha} \in H^{s-m+\nu / 2+|\alpha|}\left(X,\left.E_{2}\right|_{X}\right), \quad \alpha=\left(\alpha_{1}, \ldots, \alpha_{\nu}\right) .
$$

Next, $i_{l *}$ is the coboundary operator assigning to each element (4.3), (4.4) the sum

$$
i_{l *} v=\sum_{|\alpha|=1}^{l} v_{\alpha} \delta_{X}^{(\alpha)}
$$

of the delta function on $X$ and its derivatives of order $\leq l$ with the coefficients $v_{\alpha}$,

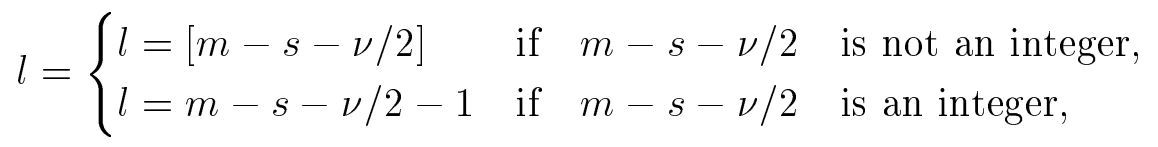

and $s<m-\nu / 2$.

Remark 4.1. If $s \geq m-\nu / 2$, then the space $H^{s-m}\left(X, E_{2}\right)$ contains no distributions supported on $X$ (a "removable singularity"), so that the equation

$$
D u=f \bmod H^{s-m}(X)
$$

is equivalent to the equation

$$
D u=f
$$

on the entire manifold $M$ for the elliptic operator $D$. There is no need to equip this operator with any boundary conditions on the submanifold $X$, and so for $s \geq m-\nu / 2$ the Sobolev problem is reduced to an elliptic operator on $M$. 
The operator in system (4.2) is given by a morphism of the special form

$$
A=\left(\begin{array}{cc}
D & i_{l *} \\
i^{*} B & 0
\end{array}\right)
$$

so that one can apply the general theory of elliptic morphisms to $A$ and find the ellipticity conditions as well as compute the index of an elliptic Sobolev problem.

This will be done in the next subsection, and here we discuss the boundary conditions in problem (4.2) in somewhat more detail. It is of special interest to find conditions under which using purely differential boundary conditions is sufficient for stating a Sobolev problem. The maximum possible order of the operator $B$ is given by the formula

$$
b_{\max }= \begin{cases}{[s-\nu / 2]} & \text { if } m-s-\nu / 2 \text { is not an integer, } \\ s-\nu / 2-1 & \text { if } m-s-\nu / 2 \text { is an integer. }\end{cases}
$$

The number of boundary conditions in a well-posed Sobolev problem must coincide with the number of the "coboundary conditions" (the dimension of the vector $v$ in (4.2)); this follows from the very form of the ellipticity conditions for morphisms. Hence Sobolev problems with purely differential boundary conditions are possible only for

$$
b_{\max } \geq l .
$$

The equality $b_{\max }=l$ is attained for $s=m / 2$.

We divide the real line $\mathbb{R} \ni s$ into the half-open intervals

$$
\left[\frac{\nu}{2}+j, \frac{\nu}{2}+j+1\right), \quad j \in \mathbb{Z}
$$

To each of these intervals there corresponds a certain type of Sobolev problems. If $s$ lies in the interval containing the point $m / 2$, then every Sobolev problem with differential conditions is equivalent to the Sobolev-Dirichlet problem, in which the values of all derivatives of order less than or equal to

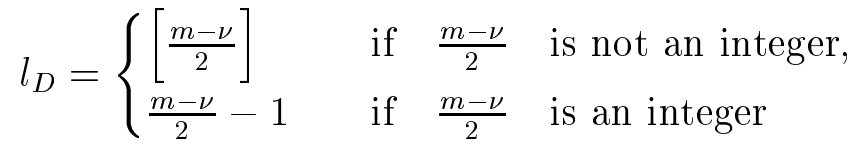

of the unknown function in transversal directions are specified on $X$.

As was already mentioned, for $s \geq m-\nu / 2$ there are no nontrivial Sobolev problems (a removable singularity).

For $s$ lying to the left of the interval containing the point $m / 2$, a well-posed Sobolev problem must necessarily contain pseudodifferential boundary conditions. 


\subsection{The Fredholm property}

Let us write out the conditions under which the morphism (4.5) defines a continuous mapping

$$
\left(\begin{array}{cc}
D & i_{l *} \\
i^{*} B & 0
\end{array}\right): \underset{H^{s_{2}}(X)}{H^{s_{1}}(M)} \rightarrow \underset{H^{\sigma_{2}}(X)}{H^{\sigma_{1}}(M)}
$$

For our problem, we have

$$
a_{g}=0, \quad a_{c}=\left\{\tau^{\alpha}\right\}, \quad a_{b}=B\left(x, p, \tau^{\prime}\right)
$$

Here $B\left(x, p, \tau^{\prime}\right)$ is a column consisting of symbols $B_{j}$ of orders $b_{j}, j=1, \ldots, J$.

In particular, $s_{2}$ and $\sigma_{2}$ in (4.8) are columns ${ }^{5}$ rather than numbers:

$$
s_{2}=\left\{s_{2}^{\alpha}\right\}_{|\alpha| \leq l}, \quad \sigma_{2}=\left\{\sigma_{2}^{j}\right\}_{j=1, \ldots, J},
$$

where $J$ is the number of boundary conditions in problem (4.1), i.e., the height of the column $a_{b}$.

With regard to this, using the continuity theorems given earlier in this paper, we see that the mapping (4.8) is a continuous operator provided that

$$
\frac{\nu}{2}+\max _{1 \leq j \leq J} b_{j}<s<m-l-\frac{\nu}{2}
$$

Let us now write out the ellipticity condition for the Sobolev problem (4.1) or, equivalently, the morphism (4.2). The first ellipticity condition in Definition 3.8 is equivalent to the ellipticity of the pseudodifferential operator $D$. Let us write out the second ellipticity condition. One can readily verify that in this case it acquires the form of the ellipticity of the pseudodifferential operator

$$
\Delta \stackrel{\text { def }}{=}-\widehat{\Phi}_{b}\left[a_{b}\right] D^{-1} \widehat{\Phi}_{c}\left[a_{c}\right]
$$

on the manifold $X$. In particular, this means that the operator (4.10) (which is a matrix operator) must be represented by a square matrix. In other words, the number $J$ of boundary conditions must be equal to the number of coboundary conditions, that is, the number of multiindices $\alpha$ such that $|\alpha| \leq l$.

Thus, the definition of ellipticity of an operator morphism results in the following notion of ellipticity for the Sobolev problem (see [Ste76]).

Definition 4.2. The Sobolev problem (4.1) is said to be elliptic if

1. the operator $D$ is an elliptic pseudodifferential operator on the manifold $M$;

2. the operator (4.10) is an elliptic pseudodifferential operator on the manifold $X$.

\footnotetext{
${ }^{5}$ In fact, one should work in the context of Douglis-Nirenberg systems [DN55].
} 
The following assertion is a straightforward consequence of the theorem on the ellipticity conditions for morphisms.

Theorem 4.3. If the Sobolev problem (4.1) is elliptic, then it is Fredholm, i.e., has a finite-dimensional kernel and a finite-dimensional cokernel.

\subsection{The Sobolev problem and the adjoint problem}

Let us now give an operator interpretation of the Sobolev problem. The problem

$$
\left\{\begin{array}{l}
D u \equiv f \quad \bmod H^{s-m}\left(M, X ; E_{2}\right), \\
i^{*} B u=g
\end{array}\right.
$$

obviously defines an operator

$$
S: H^{s}\left(M, E_{1}\right) \rightarrow\left(H^{s-m}\left(M, E_{2}\right) / H^{s-m}\left(M, X ; E_{2}\right)\right) \oplus H^{s-b-\nu / 2}(M, F),
$$

where $b$ is the order of the operator $B$.

One can also construct the adjoint of this operator.

Indeed, if we take account of the fact that $H^{s-m}\left(M, X ; E_{2}\right)$ is just the range of the coboundary operator $i_{l *}$, then the adjoint operator acts in the spaces

$$
S^{*}: \operatorname{Ker}\left(i_{l *}\right) \oplus H^{s+b+\nu / 2}(M, F) \rightarrow H^{-s}\left(M, E_{1}\right),
$$

and so the adjoint problem has the form

$$
\left\{\begin{array}{l}
D u+B i_{*} v=f \\
i_{l}^{*} u=0
\end{array}\right.
$$

where $i_{l}^{*}$ is the operator taking each function $u$ to its normal jet of order $l$ on the boundary. One can readily see that the almost inverse of the operator (4.11) has the form

$$
S^{-1}=\left(D^{-1}\left(1+i_{l *}\left(-i^{*} B D^{-1} i_{l *}\right)^{-1} i^{*} B D^{-1}\right), D^{-1} i_{l *}\left(-i^{*} B D^{-1} i_{l *}\right)^{-1}\right) .
$$

\subsection{The index formula}

Since the Sobolev problem can be reduced to an operator morphism, we obtain the following assertion by using the result of Theorem 3.18.

Theorem 4.4. Let

$$
\left\{\begin{array}{l}
D u \equiv f \quad \bmod H^{s-m}\left(M, X ; E_{2}\right), \\
i^{*} B u=g
\end{array}\right.
$$


be an elliptic Sobolev problem. Then the following index formula holds:

$$
\text { ind } S=\text { ind } D+\text { ind } \Delta \text {, }
$$

where $\Delta$ is the pseudodifferential operator

$$
\Delta=-i^{*} B D^{-1} i_{l *}
$$

Remark 4.5. One can readily see that in special coordinates the principal symbol of the pseudodifferential operator (4.13) for $p \neq 0$ is represented by the matrix with entries

$$
\begin{gathered}
{[\sigma(\Delta)]_{k j}=\left(\frac{1}{2 \pi}\right)^{\nu} \int_{\mathbb{R}^{\nu}} \frac{\tau^{k} B_{j}(x, 0, p, \tau) d \tau}{D(x, 0, p, \tau)},} \\
k=\left(k_{1}, \ldots, k_{\nu}\right), \quad j=\left(j_{1}, \quad, j_{\nu}\right), \quad|k|,|j| \leq l .
\end{gathered}
$$

Thus, we have reduced the computation of the index of the Sobolev problem to the computation of the indices of two pseudodifferential operators on smooth compact manifolds without boundary. The index formula for such operators is well known (e.g., see [AS63]).

\section{Topological Aspects of Relative Elliptic Theory}

We have already mentioned that relative elliptic theory is an elliptic theory for pairs of the form $(M, X)$, where $M$ is a smooth compact manifold and

$$
X \stackrel{i}{\hookrightarrow} M
$$

is a closed submanifold. We have seen that relative elliptic theory involves deep relationships between elliptic pseudodifferential operators on $M$ and $X$. The aim of this section is to study these relationships from the topological viewpoint. Namely, we describe a number of functors, related to elliptic operators and their symbols, from the category of manifolds with smooth embeddings as morphisms and establish Riemann-Roch type theorems comparing these functors.

\section{Preliminaries}

Let $\mathcal{K}$ be the category whose objects are smooth compact manifolds $M$ without boundary and each set $\operatorname{Hom}\left(M_{1}, M_{2}\right)$ of morphisms consists of smooth embeddings $M_{1} \hookrightarrow M_{2}$. This 
category will be referred to as the category of (smooth) embeddings. If $X, M \in \mathrm{Ob}(\mathcal{K})$ and $i: X \hookrightarrow M$ is an embedding, then there is a well-defined mapping $i_{*}: T X \longrightarrow T M$, which is also a proper embedding. (The preimage of any compact subset of $T M$ under the mapping $i_{*}$ is compact.) Using the isomorphisms

$$
T^{*} M \simeq T M, \quad T^{*} X \simeq T X
$$

(depending on the choice of Riemannian metrics on $M$ and $X$ ), we obtain a proper embedding $T^{*} X \longrightarrow T^{*} M$, which induces a mapping in the $K$-functor with compact supports, which will be denoted by

$$
i_{\text {top }}^{!}: K\left(T^{*} M\right) \longrightarrow K\left(T^{*} X\right) .
$$

The mapping (5.1) is independent of the choice of the Riemannian metrics, and we have a contravariant functor from the category $\mathcal{K}$ into the category $\mathfrak{A}$ of abelian groups; this functor takes each manifold $M$ to the $K$-group with compact supports $K\left(T^{*} M\right)$ of the cotangent bundle $T^{*} M$ and each embedding $i: X \hookrightarrow M$ to the morphism (5.1) of the corresponding $K$-groups, which will naturally be called the topological pullback.

In the subsequent sections, we use analytic tools to construct mappings that take each pseudodifferential operator on $M$ to some pseudodifferential operator on the submanifold $X$, find conditions under which elliptic operators are taken to elliptic operators, and compare the corresponding functors with the $K$-functor.

\section{The metric trace of an elliptic operator}

Consider some morphism $X \stackrel{i}{\hookrightarrow} M$ in the category $\mathcal{K}$. Suppose that the manifolds $M$ and $X$ are equipped with Riemannian metrics $\rho_{M}$ and $\rho_{X}$ such that $i^{*} \rho_{M}=\rho_{X}$. Then there is a natural embedding

$$
i_{m}: T^{*} X \longrightarrow T^{*} M,
$$

which admits a twofold description, either as the composition of the mappings

$$
T^{*} X \simeq T X \stackrel{i_{*}}{\longrightarrow} T M \simeq T^{*} M
$$

where the isomorphisms are induced by the Riemannian metric, or with the help of the orthogonal expansion

$$
\left.T^{*} M\right|_{X}=N^{*} X \oplus T^{*} X,
$$

where $N^{*} X$ is the conormal bundle of $X$ in $M$ and the orthogonality is understood in the sense of the inner product induced in the fibers of $T^{*} M$ by the Riemannian metric. One can readily see that the embedding (6.1) is a bundle monomorphism

$$
i_{m}:\left.T^{*} X \longrightarrow T^{*} M\right|_{X},
$$


so that its restriction to $T_{0}^{*} X$ can be interpreted as an embedding (denoted by the same letter)

$$
i_{m}: T_{0}^{*} X \longrightarrow T_{0}^{*} M
$$

(or, passing to the quotients with respect to the action of the group $\mathbb{R}_{+}$in the fibers, as an embedding $i_{m}: S^{*} X \longrightarrow S^{*} M$ of the cosphere bundles). Hence if

$$
D: H^{s}(M, E) \longrightarrow H^{s-m}(M, F)
$$

is an $m$ th-order pseudodifferential operator on $M$ with principal symbol

$$
\sigma(D): \pi_{M}^{*} E \longrightarrow \pi_{M}^{*} F
$$

where $\pi_{M}: T^{*} M \longrightarrow M$ is the natural projection, then

$$
i_{\mathrm{m}}^{*} \sigma(D): \pi_{X}^{*}\left(\left.E\right|_{X}\right) \longrightarrow \pi_{X}^{*}\left(\left.F\right|_{X}\right)
$$

is a well defined $m$ th-order symbol on $T_{0}^{*} X$.

Definition 6.1. The pseudodifferential operator with principal symbol (6.2) is denoted by $i_{\mathrm{m}}^{*}(D)$ and is called the metric trace of the operator $D$.

Remark 6.2. Needless to say, the operator $i_{\mathrm{m}}^{*}(D)$ is uniquely determined only modulo lower-order terms. The term "metric trace" is due to the fact that the embedding (6.1) used in the definition of the restriction of the symbol depends on the metric.

Note that if $D$ is an elliptic operator, i.e., the symbol of $D$ is an isomorphism on $T_{0}^{*} M$, then the same is true about the restriction of the symbol to $T_{0}^{*} X$. It follows that the metric trace of an elliptic operator is elliptic. Next, it is obvious that for an appropriate choice of lower-order terms the metric trace commutes with direct sums of operators and the metric trace of a continuous operator family is a continuous family. Since the set of Riemannian metrics on a given manifold is convex (and hence arcwise connected), we arrive at the following assertion.

Proposition 6.3. The metric trace is well defined as a homomorphism

$$
i_{\mathrm{m}}^{*}: \operatorname{Ell}(M) \longrightarrow \operatorname{Ell}(X)
$$

of abelian groups of classes of stable homotopy equivalence of elliptic operators on $M$ and $X$. This homomorphism is independent of the choice of Riemannian metrics on $M$ and $X$ and is functorial: if

$$
Y \stackrel{i_{X Y}}{\longrightarrow} X \stackrel{i_{M X}}{\hookrightarrow} M
$$

is a chain of embeddings and $i_{M Y}=i_{M X} \circ i_{X Y}$, then

$$
i_{M Y \mathrm{~m}}^{*}=i_{M X \mathrm{~m}}^{*} \circ i_{X Y \mathrm{~m}}^{*}
$$


Corollary 6.4. The pair $\mathrm{Ell}_{\mathrm{m}}=\left(\mathrm{Ell}, \cdot{ }_{\mathrm{m}}^{*}\right)$ is a contravariant functor from $\mathcal{K}$ to the category $\mathfrak{A}$ of abelian groups.

Now we can state the almost tautological Riemann-Roch theorem comparing the functors $K$ and $\mathrm{Ell}_{\mathrm{m}}$.

Theorem 6.5. Let $X \stackrel{i}{\hookrightarrow} M$ be an embedding of smooth compact manifolds without boundary. Then the diagram

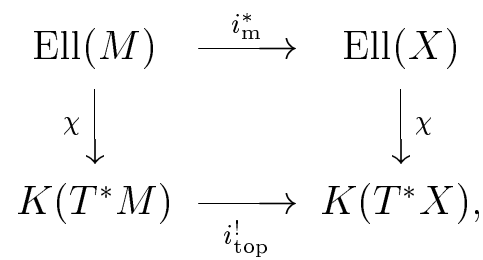

where the vertical arrows are given by the difference construction of the principal symbol of an operator, commutes.

Proof. This follows from the commutative diagram

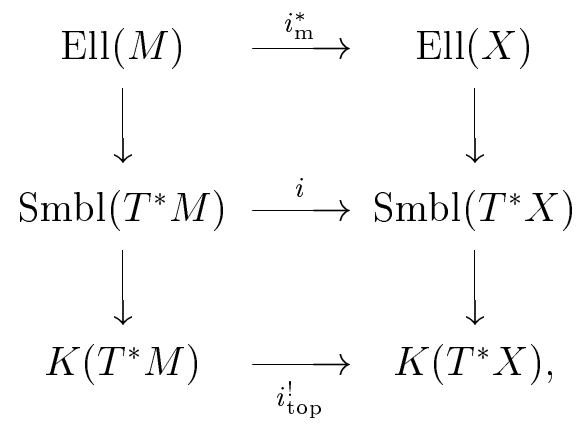

where the upper square commutes by the construction of $i_{\mathrm{m}}^{*}$ and the lower square commutes since the difference construction is natural (see [Pal65]).

\section{The pullback of an elliptic operator}

The metric trace is not the unique way of assigning a pseudodifferential operator on a submanifold to a pseudodifferential operator on a manifold. In this section, we describe the pullback related to Sobolev problems.

\subsection{The pullback of a pseudodifferential operator}

We have seen that an important role in the ellipticity condition and the index formula for elliptic morphisms is played by the noncommutative determinant, which is a pseudodifferential operator on $X$ induced by the main operator $D_{M}$ together with the boundary and 
coboundary conditions. The simplest version of the noncommutative determinant arises if one considers the elliptic morphism

$$
A=\left(\begin{array}{cc}
D & i_{*} \\
i^{*} & 0
\end{array}\right)
$$

which corresponds to the Sobolev-Dirichlet problem of order 0 for the operator $D$. The noncommutative determinant is equal to

$$
\Delta=-i_{*} D^{-1} i^{*}
$$

where $D^{-1}$ is the almost inverse of $D$. Recall that the ellipticity condition for $A$ says that both $D$ and the noncommutative determinant (7.2) must be elliptic. If this condition holds, then $A$ is Fredholm and

$$
\text { ind } A=\text { ind } D+\text { ind } \Delta \text {. }
$$

Since the boundary and coboundary conditions in the Sobolev-Dirichlet problem are standard (i.e., depend only on the embedding $i$ ), it follows that the correspondence $D \longmapsto$ $\Delta$ commutes with direct sums of operators. Using this correspondence as a model, we define a homomorphism ${ }^{6}$

$$
i_{a}^{!}: \operatorname{PSD}_{<-\nu}(M) \longrightarrow \operatorname{PSD}(X)
$$

by setting

$$
i_{a}^{!}(D)=i^{*} \circ D \circ i_{*}
$$

Here $\operatorname{PSD}(X)$ is the abelian semigroup (with respect to direct sum) of pseudodifferential operators on $X$ and $\operatorname{PSD}_{<-\nu} \subset \operatorname{PSD}(M)$ is the subsemigroup of operators of order $<-\nu$ $(\nu=\operatorname{codim} X)$. As follows from the results of the first part, the homomorphism (7.4)(7.5) is well defined, and moreover, the principal symbol of the operator (7.5) can be obtained from the principal symbol of $D$ by integrating over the fibers of the conormal bundle. In special local coordinates, this formula reads

$$
\sigma\left(i_{a}^{!}(D)\right)(x, p)=\left(\frac{1}{2 \pi}\right)^{\nu} \int_{\mathbb{R}^{\nu}} \sigma(D)(x, 0, p, \tau) d \tau .
$$

\subsection{The regularized pullback}

The pullback thus defined has two disadvantages from the viewpoint of our goals. First, it is well defined only for operators of sufficiently large negative order (depending on the codimension of the subspace). Second, we shall see below that the operator (7.5) need not be elliptic if $D$ is elliptic. It turns out that both disadvantages can be eliminated

\footnotetext{
${ }^{6}$ Which differs from the noncommutative determinant in the Sobolev-Dirichlet problem only in sign and the absence of the exponent ${ }^{-1}$ on $D$.
} 
by the following regularization. Let $\Delta_{M}$ and $\Delta_{X}$ be the Laplace operators on $M$ and $X$, respectively, with respect to the given Riemannian metrics. We set

$$
i_{a}^{!}(D)(k)=\left(1-\Delta_{X}\right)^{k-\nu / 2} i_{a}^{!}\left(\left(1-\Delta_{M}\right)^{-k} D\right) . \quad k \in \mathbb{R} .
$$

(If $D$ acts in sections of vector bundles, then one should take Laplacians with coefficients in these bundles.) The operator (7.7) is well defined for sufficiently large $k$, namely, for

$$
k>\frac{\nu+\operatorname{ord} D}{2},
$$

where ord $D$ is the order of the operator $D$. The values of $k$ satisfying this inequality will be referred to as admissible.

Remark 7.1. We have included the factor $\left(1-\Delta_{X}\right)^{k-\nu / 2}$ in the definition of regularization so as to normalize orders: with this definition, the order of the pullback of an operator coincides with the order of the operator itself. This is not necessary in principle, but useful, as we shall see in Remark 8.6.

Theorem 7.2. Let $D$ be an elliptic operator. Then for all sufficiently large $k$ the operator $i_{a}^{!}(D)(k)$ defined in $(7.7)$ is also elliptic, and moreover, its equivalence class in $\operatorname{Ell}(X)$ is independent of $k$ and is uniquely determined by the equivalence class of $D$ in $\operatorname{Ell}(M)$.

Proof. Without loss of generality, we can assume (including sufficiently many factors $(1+\Delta)^{-1}$ in $\left.D\right)$ that $D$ itself has a sufficiently large negative order, so that its symbol is bounded and integrable at infinity. The formula for the principal symbol of the operator of the operator $i_{a}^{!}(D)(k)$ on the sphere $|p|=1$ (where $|p|$ is the natural norm on the fibers of $\left.T^{*} X\right)$ in special coordinates has the form

$$
\sigma\left(i_{a}^{!}(D)(k)\right)=\left(\frac{1}{2 \pi}\right)^{\nu} \int_{\mathbb{R}^{\nu}} \frac{\sigma(D)(x, 0, p, \tau)}{\left(1+\tau^{2}\right)^{k}} d \tau .
$$

$\left(\right.$ Here $\left.\tau^{2}=\tau_{1}^{2}+\cdots+\tau_{\nu}^{2}.\right)$ We set

$$
c_{k \nu}=\int_{\mathbb{R}^{\nu}} \frac{d \tau}{\left(1+\tau^{2}\right)^{k}} .
$$

Then the sequence $\left(c_{k \nu}\left(1+\tau^{2}\right)^{k}\right)^{-1}$ converges to the delta function as $k \rightarrow \infty$, and so

$$
c_{k \nu}^{-1} \sigma\left(i_{a}^{!}(D)(k)\right)(x, p) \rightrightarrows\left(\frac{1}{2 \pi}\right)^{\nu} \sigma(D)(x, 0, p, 0) \neq 0 .
$$

(The uniform convergence with respect to $(x, p) \in S^{*} X$ can readily be established.) This proves the desired assertion.

Definition 7.3. By $i_{\text {reg }}^{!}(D) \in \operatorname{Ell}(X)$ we denote the class defined in the situation of Theorem 7.2 by the operator $i_{a}^{!}(D)(k)$ for sufficiently large $k$.

Remark 7.4. One can show that $i_{a}^{!}(D)(z)$ is a meromorphic operator-valued function of $z$. It is of interest to study the residues of this function, but we do not touch this topic in the present paper. 


\subsection{The Riemann-Roch theorem for the regularized pullback}

Now we can establish a Riemann-Roch theorem comparing the functors $K$ and $\operatorname{Ell}_{\text {reg }}=$ $($ Ell, $\cdot$ reg $)$.

Theorem 7.5. Let $X \stackrel{i}{\hookrightarrow} M$ be an embedding of smooth compact manifolds without boundary. Then the diagram

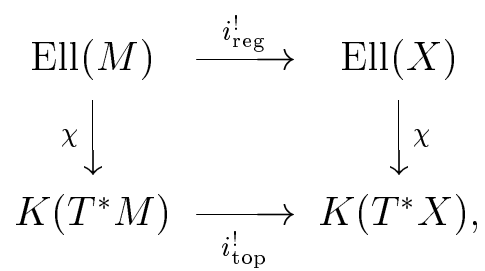

where the vertical arrows are given by the difference construction of the principal symbol of an operator, commutes.

Proof. It follows from formula (7.11) that $i_{\text {reg }}^{!}=i_{\mathrm{m}}^{*}$. It remains to apply Theorem 6.5.

\section{A finitely regularized pullback}

The definition of the regularized pullback has the following peculiarity: one does not simply choose a $k$ such that the integral in the definition of the pullback converges (an admissible $k$ ); instead, one must take an arbitrary sufficiently large $k$. It is only under that condition that Theorem 7.2 guarantees that a given elliptic operator $D$ produces an elliptic operator and Theorem 7.5 compares the two functors. The following natural question arises: is it possible (say, for operators of given order) to use some given finite regularization, i.e., choose an admissible $k$ once and for all? Then we would have the

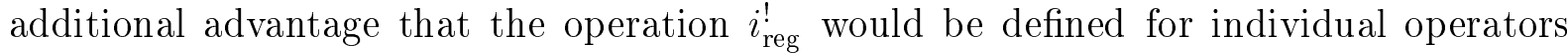
rather than only for their equivalence classes in $\operatorname{Ell}(M)$.

The following example shows that the answer is "no." Later on in this paper, we shall indicate operator classes for which one can successfully use a finite regularization.

\subsection{A counterexample}

In the forthcoming argument, we analyze the behavior of symbols over a given point $x \in X$. One can readily obtain specific counterexamples by considering, say, operators with constant coefficients on the tori $T^{m-\nu} \hookrightarrow T^{m}$.

Thus, let $\mathbb{R}^{m}=\mathbb{R}^{n} \oplus \mathbb{R}^{\nu}$ be the $m$-dimensional space with coordinates $(p, \tau), p \in \mathbb{R}^{n}$, $\tau \in \mathbb{R}^{\nu}$. We shall study the pullback (7.9) of a symbol $a(p, \tau)$ of arbitrary given order $r$ for a given admissible $k$. Without loss of generality, we can assume that $k=0$ (including the corresponding nonzero factor in $a$ ) and $r<-\nu$. For simplicity, we work with scalar symbols, which suffices for constructing a counterexample. 
Proposition 8.1. There exists an elliptic symbol $a(p, \tau)$ of order $r$ such that $i_{\mathrm{m}}^{*}(a)=1$ and the pullback $i_{a}(a)$ is homotopic in the class of elliptic symbols to an arbitrarily chosen elliptic symbol $b(p)$ on the sphere $S^{n-1} \subset \mathbb{R}^{n}$.

Remark 8.2. Prior to proving the proposition, note that it also implies the existence of an elliptic symbol whose pullback under a finite regularization is not elliptic. Indeed, take a symbol $b$ nonhomotopic to unity and for the corresponding symbol $a$ consider the family $\left(1+p^{2}+\tau^{2}\right)^{-\alpha} a(p, \tau)$. For $\alpha=0$, the pullback of this symbol is homotopic to $b$ in the class of elliptic symbols, and as $\alpha \rightarrow \infty$, the pullback becomes homotopic to the metric trace, i.e., to unity. Thus, somewhere in between the pullback is necessarily not elliptic.

However, the existence of an elliptic symbol with nonelliptic pullback can be shown much simpler, by using a second-order differential operator as an example. Namely, let $D$ be the square of the "distorted" Cauchy-Riemann operator:

$$
D=\left(\frac{\partial}{\partial x}+i \lambda \frac{\partial}{\partial y}\right)^{2},
$$

where $\lambda$ is a nonzero real constant to be chosen later. Then $\sigma(D)=-(p+i \lambda \tau)^{2}$, and on the sphere $\{|p|=1\}$, i.e., for $p= \pm 1$, we have

$$
\begin{aligned}
\sigma\left(i_{a}^{!}(D)(k)\right) & =-\left(\frac{1}{2 \pi}\right) \int_{-\infty}^{\infty} \frac{d \tau}{\left(1+\tau^{2}\right)^{k}}-\frac{i \lambda}{\pi} \int_{-\infty}^{\infty} \frac{\tau d \tau}{\left(1+\tau^{2}\right)^{k}}+\frac{\lambda^{2}}{2 \pi} \int_{-\infty}^{\infty} \frac{\tau^{2} d \tau}{\left(1+\tau^{2}\right)^{k}} \\
& =-\frac{1}{2 \pi}\left[c_{k 1}-\left(c_{k-1,1}-c_{k 1}\right) \lambda^{2}\right] .
\end{aligned}
$$

(The term of first order in $\tau$ disappears after the integration, since the integrand is odd.) For given $k$, we obtain a pullback with zero principal symbol by setting

$$
\lambda=\sqrt{\frac{c_{k 1}}{c_{k-1,1}-c_{k 1}}} .
$$

Proof of the proposition. We represent the sphere

$$
S^{m-1}=\left\{p^{2}+\tau^{2}=1\right\}
$$

as the union

$$
S^{m-1}=\bigcup_{\omega \in S^{n-1}} D_{\omega}
$$

of the subsets

$$
D_{\omega}=\left\{(p, \tau) \in S^{m-1}|p=| p \mid \omega\right\},
$$

homeomorphic to the $\operatorname{disc}\{|\tau| \leq 1\}$. All these subsets intersect in the common sphere

$$
S^{\nu-1}=\partial D_{\omega}=\{p=0,|\tau|=1\} .
$$


The formula for the symbol of the pullback on the sphere $S^{n-1}$ acquires the form

$$
i_{a}^{!}(a)(\omega)=\int_{D_{\omega}} a(p, \tau) \mu(\tau) d \tau,
$$

where $\mu(\tau)$ is a continuous function in the disk $\{|\tau| \leq 1\}$ and is strictly positive outside the boundary of the disk. (This function, which depends on the order $r$ of the symbol $a$, is easy to compute, but the specific expression will not be needed here.)

Let $\varepsilon>0$ be a small positive number. We set

$$
\begin{aligned}
& D_{\omega, \varepsilon}=D_{\omega} \cap\{\varepsilon \leq|\tau| \leq 1-\varepsilon\}, \\
& D_{\omega, \varepsilon}^{+}=D_{\omega, \varepsilon} \cap\left\{\tau_{1}>0\right\}, \\
& D_{\omega, \varepsilon}^{-}=D_{\omega, \varepsilon} \cap\left\{\tau_{1}<0\right\} .
\end{aligned}
$$

We define a continuous symbol $a(p, \tau)$ on the sphere $S^{m-1}$ by the following conditions, which determine its behavior on each of the sets $D_{\omega}$ :

1. $a$ is elliptic (i.e., everywhere nonzero on the sphere) and nowhere exceeds 1 in modulus;

2. $a=1$ on $\partial D_{\omega}$;

3. $\arg a=\arg b(\omega)$ in $D_{\omega, \varepsilon}^{+}$for $\omega_{1} \geq-\varepsilon / 2$;

4. $a=e^{i \arg b(\omega)}$ in $D_{\omega, \varepsilon}^{+}$for $\omega_{1} \geq 0$;

5. $|a|<\varepsilon$ in $D_{\omega, \varepsilon}^{+}$for $\omega_{1}<-\varepsilon / 2$;

6. $\arg a=\arg b(\omega)$ in $D_{\omega, \varepsilon}^{-}$for $\omega_{1} \leq \varepsilon / 2$;

7. $a=e^{i \arg b(\omega)}$ in $D_{\omega, \varepsilon}^{-}$for $\omega_{1} \leq 0$;

8. $|a|<\varepsilon$ in $D_{\omega, \varepsilon}^{-}$for $\omega_{1}>\varepsilon / 2$.

The existence of a symbol satisfying these conditions is clear, since the sets $\omega_{1} \geq-\varepsilon, \omega_{1} \leq$ $\varepsilon$, etc. are contractible. Condition (2) guarantees that $i_{\mathrm{m}}^{*}(a)=1$. Next, for sufficiently small $\varepsilon>0$ the main contribution to the integral (8.1) is from the domains $D_{\omega, \varepsilon}^{+}$for $\omega_{1} \geq$ $-\varepsilon$ and $D_{\omega, \varepsilon}^{-}$for $\omega_{1} \leq \varepsilon$. It follows that the integral is nonzero and $\arg i_{a}^{!} a(\omega) \rightarrow \arg b(\omega)$ as $\varepsilon \rightarrow 0$. Hence, for sufficiently small $\varepsilon>0$ the symbols $i_{a}^{!} a(\omega)$ and $b(\omega)$ are homotopic in the class of elliptic symbols, as desired. 


\subsection{The pullback for linear principal symbols}

However, there are two cases in which the pullback of an elliptic operator is elliptic even for a finite regularization (7.7) (i.e., for any admissible $k$ ). These cases are considered in this subsection and the next subsection.

Definition 8.3. Let $M$ be a Riemannian manifold. We say that $D$ is a pseudodifferential operators with linear principal symbol on $M$ if for each point $y \in M$ the restriction of the principal symbol $\sigma(D)$ to the unit sphere $S_{y}^{*} M \subset T_{y}^{*} M$ coincides with the restriction to the same sphere of some homogeneous linear function on $T_{y}^{*} M$.

Remark 8.4. This notion of an operator with linear principal symbol depends on the choice of unit spheres, i.e., of the Riemannian metric, with the exception of the operators whose principal symbol is linear on the entire $T_{y}^{*} M$ (say, first-order differential operators). The reason for introducing this class of operators is as follows: this class, in contrast with the class of first-order differential operators, is preserved by pullbacks regardless of the normalization. (However, see Remark 8.6 below.) Differential operators are undoubtedly of main interest in specific examples.

Theorem 8.5. Let $X \stackrel{i}{\hookrightarrow} M$ be an embedding of Riemannian manifolds, and let $D$ be an elliptic pseudodifferential operator with linear principal symbol on $M$. Then for each admissible $k$ the pullback $i_{a}^{!}(D)(k)$ is an elliptic pseudodifferential operator with linear principal symbol on $X$ homotopic in the class of elliptic operators to the metric trace $i_{\mathrm{m}}^{*}(D)$ (which is also an elliptic pseudodifferential operator with linear principal symbol on $X)$.

Proof. In special coordinates, the principal symbol $\sigma(D)$ has the form

$$
\sigma(D)=(A(x, t) p+B(x, t) \tau)\left(|p|^{2}+\tau^{2}\right)^{\alpha},
$$

where $\alpha=\frac{1}{2}(\operatorname{ord} D-1)$,

$$
A(x, t)=\left(A_{1}(x, t), \ldots, A_{m}(x, t)\right), \quad B(x, t)=\left(B_{1}(x, t), \ldots, B_{\nu}(x, t)\right)
$$

are some sets of matrices, $A p=\sum A_{j} p_{j}$, and similarly for $B \tau$. On the sphere $\{|p|=1\}$, the principal symbols of the metric trace and the regularized pullback of $D$ (for admissible values of the regularization parameter $k$ ) have the form

$$
\begin{gathered}
\sigma\left(i_{\mathrm{m}}^{*}(D)\right)=i_{\mathrm{m}}^{*} \sigma(D)=A(x, 0) p, \\
\sigma\left(i_{a}^{!}(D)(k)\right)=\left(\frac{1}{2 \pi}\right)^{\nu} c_{k-\alpha, \nu} A(x, 0) p .
\end{gathered}
$$

(Here, just as in the example in Remark 8.2, the term with $B \tau$ disappears, since the integrand is odd.) The expressions (8.3) and (8.4) coincide up to a nonzero factor; the fact that the metric trace is elliptic has already been discussed. The assertion of the theorem now follows readily. 
Remark 8.6. One can readily see that with our normalizations (see Remark 7.1) the pullback of a first-order differential operator is (up to lower-order terms) again a firstorder differential operator.

By abuse of notation, we denote the pullback of an elliptic operator $D$ with linear principal symbol by $i_{a}^{!}(D)$, omitting the regularization parameter $k$. This is justified, since by the theorem the pullback defines the same element in $\operatorname{Ell}(X)$ for any admissible $k$. The above theorem implies the following important assertion, which can be treated as the Riemann-Roch theorem for operators with linear principal symbol.

Corollary 8.7. If $D$ is an elliptic operator with linear principal symbol, then

$$
\left[i_{a}^{!}(D)\right]=i_{\mathrm{top}}^{!} \sigma(D) \in K\left(T^{*} X\right)
$$

where the square brackets stand for the difference construction of the principal symbol.

\subsection{Pullbacks and exterior tensor products}

Another case in which the ellipticity of the pullback is guaranteed is the case in which the elliptic operator in question has the structure of an exterior tensor product in a neighborhood of $X$. The exterior tensor product, which takes two elliptic operators $A_{1}$ and $A_{2}$ on two manifolds to an elliptic operator $A_{1} \# A_{2}$ on the product of these manifolds (or on the bundle, where one of the manifolds is the base and the other is the fiber) plays a fundamental role in elliptic theory. We shall describe this construction in the situation of an embedding $X \stackrel{i}{\hookrightarrow} M$, which is of interest to us. We identify a tubular neighborhood $U$ of $X$ with a neighborhood of the zero section in the normal bundle $N X$ via the exponential mapping.

Let

$$
D_{1}: C^{\infty}\left(X, E_{1}\right) \longrightarrow C^{\infty}\left(X, F_{1}\right)
$$

be an elliptic differential ${ }^{7}$ operator of some order $m$ on $X$. Next, let

$$
D_{2}: C^{\infty}\left(N_{x} X, E_{2 x}\right) \longrightarrow C^{\infty}\left(N_{x} X, F_{2 x}\right)
$$

be an elliptic differential operator of the same order $m$ in the fiber $N_{x}$ smoothly depending on the parameter $x \in X$ and defined at least in $U$. Here $E_{2 x}$ and $F_{2 x}$ are the restrictions to $N_{x}$ of some vector bundles $E_{2}$ and $F_{2}$ over $U$. We assume that all bundles in question are Hermitian. The lifts of $E_{1}$ and $F_{1}$ to $U$ via the natural projection of the normal bundle on the base $X$ will be denoted by the same letters.

\footnotetext{
${ }^{7}$ We work only with differential operators to avoid considering nonsmooth symbols. The results remain valid for pseudodifferential operators if for operators with nonsmooth symbols one uses definitions in the spirit of [Hör83b].
} 
Definition 8.8. The exterior tensor product of the operator $D_{1}$ by the operator family $D_{2}$ (or, for brevity, of the operators $D_{1}$ and $D_{2}$ ) is the differential operator

$$
D_{1} \# D_{2}: C^{\infty}\left(U, E_{1} \otimes E_{2} \oplus F_{1} \otimes F_{2}\right) \longrightarrow C^{\infty}\left(U, F_{1} \otimes E_{2} \oplus E_{1} \otimes F_{2}\right)
$$

in $U$ with principal symbol

$$
\sigma\left(D_{1} \# D_{2}\right)=\left(\begin{array}{cc}
\sigma\left(D_{1}\right) \otimes 1_{\pi^{*} E_{2}} & 1_{\pi^{*} F_{1}} \otimes \sigma\left(D_{2}\right)^{*} \\
1_{\pi^{*} E_{1}} \otimes \sigma\left(D_{2}\right) & -\sigma\left(D_{1}\right)^{*} \otimes 1_{\pi^{*} F_{2}}
\end{array}\right)
$$

where $\pi: T_{0}^{*} M \longrightarrow M$ is the natural projection. (In formula (8.9), the symbol $\sigma\left(D_{1}\right)$ is lifted from $T^{*} X$ to $T^{*}(N X)$ with the help of the projection

$$
T^{*}(N X) \simeq T(N X) \longrightarrow T X \simeq T^{*} X
$$

where the isomorphisms are induced by the Hermitian metric.)

Theorem 8.9. Let $D$ be an mth-order elliptic operator on $M$ coinciding on $X$ with the exterior tensor product (8.8):

$$
\left.D\right|_{X}=\left.\left(D_{1} \# D_{2}\right)\right|_{X}
$$

Then for any admissible $k$ the pullback of $D$ on $X$ is elliptic, and

$$
i_{a}^{!}(D)(k)=i_{\mathrm{m}}^{*}(D) \in \operatorname{Ell}(X) .
$$

Thus, the pullback is independent of the regularization parameter $k$ up to homotopy equivalence, and so it will be denoted by $i_{a}^{!}(D)$ in what follows.

The following theorem can be viewed as a "Riemann-Roch theorem for exterior tensor products."

Theorem 8.10. Under the assumptions of the preceding theorem, the formula

$$
\left[i_{a}^{!}(D)\right]=\left[D_{1}\right]\left(\left[E_{2 X}\right]-\left[F_{2 X}\right]\right) \in K\left(T^{*} X\right)
$$

holds, where $[A]$ is the difference construction of the principal symbol of an operator $A$, $[E]$ is the element generated by a bundle $E$ in the $K$-group, and $E_{2 X}$ and $F_{2 X}$ are the restrictions of the bundles $E_{2}$ and $F_{2}$ to $X$.

Proof of theorems 8.9 and 8.10. Let us compute the symbols of the metric trace and the pullback of $D$ on $S^{*} X$. We have

$$
\sigma\left(i_{\mathrm{m}}^{*}\left(D_{1} \# D_{2}\right)\right)=\left(\begin{array}{cc}
\sigma\left(D_{1}\right)(p) \otimes 1_{\pi^{*} E_{2 X}} & 0 \\
0 & -\sigma\left(D_{1}^{*}\right)(p) \otimes 1_{\pi^{*} F_{2 X}}
\end{array}\right) .
$$

On the other hand,

$$
\sigma\left(i_{a}^{!}\left(D_{1} \# D_{2}\right)\right)(k)=\left(\begin{array}{cc}
c_{k \nu} \sigma\left(D_{1}\right)(p) \otimes 1_{\pi^{*} E_{2 X}} & 1_{\pi^{*} F_{1 X}} \otimes V \\
1_{\pi^{*} E_{1 X}} \otimes V^{*} & -c_{k \nu} \sigma\left(D_{1}^{*}\right)(p) \otimes 1_{\pi^{*} F_{2 X}}
\end{array}\right),
$$


where

$$
V=\int \frac{\sigma\left(D_{2}\right)(\tau) d \tau}{\left(1+\tau^{2}\right)^{k}}
$$

The symbol (8.13) is obviously elliptic (its matrix is the exterior tensor product of the elliptic symbol $c_{k \nu} \sigma\left(D_{1}\right)(p)$ by the matrix $\left.V\right)$ and homotopic in the class of elliptic symbols to the matrix (8.12) via the homotopy

$$
\left(\begin{array}{cc}
{\left[(1-t) c_{k \nu}+t\right] \sigma\left(D_{1}\right)(p) \otimes 1_{\pi^{*} E_{2 X}}} & (1-t) 1_{\pi^{*} F_{1 X}} \otimes v(k) \\
(1-t) 1_{\pi^{*} E_{1 X}} \otimes \bar{v}(k) & -\left[(1-t) c_{k \nu}+t\right] \sigma\left(D_{1}^{*}\right)(p) \otimes 1_{\pi^{*} F_{2 X}}
\end{array}\right),
$$

which proves the assertion of the first theorem. To prove the second theorem, one should explicitly compute the element corresponding to the symbol (8.12) in the $K$-group with regard to the fact that $\left[D_{1}^{*}\right]=-\left[D_{1}\right]$. The proof is complete.

\section{The pullback of geometric operators}

In this section, we compute the pullback for the Hirzebruch, Euler, Todd (CauchyRiemann), and Dirac operators. All these operators are linear and can be represented as exterior tensor products in neighborhoods of submanifolds; thus, they are covered by the assumptions of both Subsections 8.2 and 8.3. Hence their pullbacks are elliptic for any admissible value of the regularization parameter, and the corresponding element of the $K$-group can be computed according to Theorem 8.10. Geometric operators are a special case of group operators (operators associated with the $G$-structure of a manifold). We briefly recall this well-known construction mainly to introduce the notation. Details can be found, say, in [Pal65].

\subsection{Group operators}

Let $G$ be a compact Lie group and $M$ a compact manifold without boundary.

Definition 9.1. One says that $M$ is equipped with a $G$-structure if an orientationpreserving isomorphism

$$
P \underset{G}{\times} V \simeq T M
$$

is given, where $V$ is an oriented real $G$-module of dimension $n=\operatorname{dim} M$ and $P \longrightarrow M$ is a principal $G$-bundle. (In other words, $T M$ is equipped with the structure of a vector bundle with structure group $G$ associated with the principal bundle $P$.)

Let $M$ be a manifold with $G$-structure (9.1), and let $\widetilde{E}$ and $\widetilde{F}$ be finite-dimension complex $G$-modules. Next, let

$$
\sigma: S\left(V^{*}\right) \longrightarrow \operatorname{Hom}(\widetilde{E}, \widetilde{F})
$$


be a $G$-equivariant $\left(\sigma(g \xi)=g \sigma(\xi) g^{-1}\right)$ mapping such that $\sigma(\xi)$ is invertible for all $\xi \in$ $S\left(V^{*}\right)$. (Here $S\left(V^{*}\right)$ is the unit sphere in $V^{*}$.) The mapping (9.2) determines an elliptic symbol

$$
\sigma_{P}: \pi^{*} E \longrightarrow \pi^{*} F
$$

on the cosphere bundle $S^{*} M$, where

$$
E=P \underset{G}{\times} \widetilde{E}, \quad F=P \underset{G}{\times} \widetilde{F},
$$

and $\pi: S^{*} M \longrightarrow M$ is the natural projection.

Definition 9.2. The operator $D$ on $M$ with symbol $\sigma(D)=\sigma_{P}$ is called the group operator (G-operator) on $M$ associated with the principal bundle $P$ and the mapping $\sigma$. The method used to construct $D$ from these data is referred to as the universal construction.

We also consider group operators with coefficients in an arbitrary vector bundle $H$. The symbol of such an operator is obtained as the tensor product of the symbol of a group operator by the identity automorphism $1_{H}$.

\subsection{The Euler and Hirzebruch operators}

Let $V$ be a real vector space of $\operatorname{dimension} \operatorname{dim} V=n$ equipped with an inner product. We treat $V$ as an oriented $S O(n)$-module. (To this end, it suffices to choose an orthonormal basis $\varepsilon_{1}, \ldots, \varepsilon_{n}$ in $V$.) By

$$
\Lambda_{\mathbb{C}}(V)=\bigoplus_{j=0}^{n} \Lambda^{j}(V) \otimes \mathbb{C}
$$

we denote the complexified direct sum of exterior powers of $V$. This is also an $S O(n)$ module with respect to the action defined on decomposable vectors by the natural formula

$$
g\left(f_{1} \wedge \cdots \wedge f_{s}\right) \stackrel{\text { def }}{=} g\left(f_{1}\right) \wedge \cdots \wedge g\left(f_{s}\right), \quad g \in S O(n), \quad f_{j} \in V .
$$

(The action is extended to the entire $\Lambda_{\mathbb{C}}(V)$ by linearity.)

We define an $S O(n)$-equivariant mapping

$$
\sigma: S\left(V^{*}\right) \longrightarrow \operatorname{Hom}\left(\Lambda_{\mathbb{C}}(V), \Lambda_{\mathbb{C}}(V)\right)
$$

by the formula

$$
\sigma(\xi)=\xi \wedge+i_{\xi}
$$

where $i_{\xi}$ is the operator of interior multiplication by a vector $\xi$, i.e., the substitution of $\xi$ as the first argument into a form (e.g., see [Stb64]). (We have used the identification $V^{*} \simeq V$.) 
An oriented Riemannian manifold $M$ of dimension $n$ can always be equipped with an $S O(n)$-structure. (The structure group of the tangent bundle is reduced to $S O(n)$.) The universal construction applied to the map (9.4)-(9.5) gives the elliptic operator

$$
d+\delta: \Gamma\left(\Lambda_{\mathbb{C}}\left(T^{*} M\right)\right) \longrightarrow \Gamma\left(\Lambda_{\mathbb{C}}\left(T^{*} M\right)\right)
$$

where

$$
\Lambda_{\mathbb{C}}\left(T^{*} M\right)=\bigoplus_{j=0}^{n} \Lambda^{j} T^{*} M \otimes_{\mathbb{R}} \mathbb{C}
$$

is the complexified bundle of exterior forms of all degrees on $M$ and $\delta$ is the adjoint of the exterior differentiation operator with respect to the Riemannian metric. The Euler and Hirzebruch operators are obtained from the operator (9.6) by restriction to some subspaces (or, equivalently, by restriction of the symbol (9.4)-(9.5) to some submodules of $\left.\Lambda_{\dot{\mathbb{C}}}(V)\right)$.

\subsubsection{The Hirzebruch operator}

Suppose that the module $V$ (and, accordingly, the manifold $M$ ) is even-dimensional: $n=2 N$. Then $\Lambda_{\mathbb{C}}(V)$ has the involution

$$
\alpha=i^{j(j-1)+N} *: \Lambda_{\mathbb{C}}(V) \longrightarrow \Lambda_{\mathbb{C}}(V),
$$

which commutes with the group action. Here $*$ is the Hodge operator given by the formula (e.g., see [Rha55])

$$
*\left(\varepsilon_{\beta_{1}} \wedge \cdots \wedge \varepsilon_{\beta_{k}}\right)=(-1)^{\sigma(\beta)} \varepsilon_{\beta_{k+1}} \wedge \cdots \wedge \varepsilon_{\beta_{2 N}},
$$

where $\beta=\left\{\beta_{1}, \ldots, \beta_{2 N}\right\}$ is a permutation of the numbers $1, \ldots, 2 N, \sigma(\beta)$ is the parity of $\beta$, and $i^{j(j-1)+N}$ stands for the operator acting by multiplication by the number $i^{j(j-1)+N}$ (here $i$ is the imaginary unit) on the component $\Lambda_{\mathbb{C}}^{j}(V)$. Thus, we have the direct sum of modules

$$
\Lambda_{\mathbb{C}}(V)=\Lambda_{+}(V) \oplus \Lambda_{-}(V),
$$

where the $\Lambda_{ \pm}(V)$ are the eigenspaces of $\alpha$ corresponding to the eigenvalues \pm 1 .

The homomorphism (9.4) anticommutes with $\alpha$ for any $\xi$ :

$$
\sigma(\xi) \alpha+\alpha \sigma(\xi)=0
$$

Hence the restriction

$$
\sigma_{\mathcal{H}}=\left.\sigma\right|_{\Lambda_{+}(V)}: \Lambda_{+}(V) \longrightarrow \Lambda_{-}(V)
$$

is well defined. An application of the universal construction to $\sigma_{\mathcal{H}}$ gives the operator

$$
\mathcal{H}_{M}=d+\delta: \Gamma\left(\Lambda_{+}\left(T^{*} M\right)\right) \longrightarrow \Gamma\left(\Lambda_{-}\left(T^{*} M\right)\right)
$$


which is called the Hirzebruch operator on $M$. Here

$$
\Lambda_{ \pm}\left(T^{*} M\right) \subset \Lambda_{\mathbb{C}}\left(T^{*} M\right)
$$

are the bundles on $M$ associated with the modules $\Lambda_{ \pm}(V)$. The principal symbol of the Hirzebruch operator will be denoted by $\sigma\left(\mathcal{H}_{M}\right)$. The Hirzebruch operator with coefficients in a bundle $E$ over $M$ will be denoted by $\mathcal{H}_{M} \otimes 1_{E}$; its principal symbol is $\sigma\left(\mathcal{H}_{M}\right) \otimes 1_{\pi^{*} E}$.

Now let $X \stackrel{i}{\hookrightarrow} M$ be an oriented Riemannian submanifold of even dimension $m=2 l$. On $X$ we have a direct sum decomposition of the cotangent bundle:

$$
\left.T M\right|_{X}=T X \oplus N X .
$$

One has (e.g., see [Pal65])

$$
\begin{aligned}
\Lambda_{+}\left(\left.T M\right|_{X}\right) & =\left(\Lambda_{+}(T X) \otimes \Lambda_{+}(N X)\right) \oplus\left(\Lambda_{-}(T X) \otimes \Lambda_{-}(N X)\right), \\
\Lambda_{-}\left(T M_{X}\right) & =\left(\Lambda_{-}(T X) \otimes \Lambda_{+}(N X)\right) \oplus\left(\Lambda_{+}(T X) \otimes \Lambda_{-}(N X)\right) .
\end{aligned}
$$

Moreover, a straightforward computation shows that, according to (9.14)-(9.15), the Hirzebruch operator can be represented on $X$ as the exterior tensor product

$$
\left.\mathcal{H}_{M}\right|_{X}=\left.\left(\mathcal{H}_{X} \# \mathcal{H}_{N X}\right)\right|_{X}
$$

of the Hirzebruch operator on $X$ by the family

$$
\mathcal{H}_{N X}=\left\{\mathcal{H}_{N_{x} X}\right\}_{x \in X}
$$

of Hirzebruch operators in the fibers of the normal bundle $N X$. By applying Theorem 8.10, we arrive at the following assertion.

Theorem 9.3. The pullback on $X$ of the Hirzebruch operator on $M$ satisfies the following relation in $K\left(T^{*} X\right)$ :

$$
\left[i_{a}\left(\mathcal{H}_{M}\right)\right]=\left[\mathcal{H}_{X}\right]\left(\left[\Lambda_{+}(N X)\right]-\left[\Lambda_{-}(N X)\right]\right) \in K\left(T^{*} X\right) .
$$

Remark 9.4. 1. By Theorem 8.9, the same relation naturally holds for the metric trace of the Hirzebruch operator.

2. Let $E$ be an arbitrary vector bundle over $M$. Since

$$
\left(\mathcal{H}_{X} \# \mathcal{H}_{N X}\right) \otimes 1_{E}=\mathcal{H}_{X} \#\left(\mathcal{H}_{N X} \otimes 1_{E}\right),
$$

where the second factor in the exterior tensor product is the family given by the formula

$$
\mathcal{H}_{N X} \otimes 1_{E} \stackrel{\text { def }}{=}\left\{\left.\mathcal{H}_{N_{x} X} \otimes 1_{E}\right|_{N_{x} X}\right\}_{x \in X},
$$

we arrive at the following formula for the pullback of the Hirzebruch operator with coefficients in $E$ :

$$
\left[i_{a}^{!}\left(\mathcal{H}_{M} \otimes 1_{E}\right)\right]=\left[\mathcal{H}_{X}\right]\left(\left[\Lambda_{+}(N X) \otimes E\right]-\left[\Lambda_{-}(N X) \otimes E\right]\right) \in K\left(T^{*} X\right) .
$$




\subsubsection{The Euler operator}

Now we do not assume that the module $V$ and the manifold $M$ are even-dimensional (even though the results are actually of interest only for the even-dimensional case) and replace (9.10) by the decomposition

$$
\Lambda_{\mathbb{C}}(V)=\Lambda_{\text {odd }}(V) \oplus \Lambda_{\text {even }}(V),
$$

where $\Lambda_{\text {odd }}(V)$ and $\Lambda_{\text {even }}(V)$ are the subspaces of complex-valued forms of odd and even degrees, respectively.

The restriction of the symbol $(9.4)-(9.5)$ to $\Lambda_{\text {even }}(V)$ acts in the spaces

$$
\sigma_{\mathcal{E}}=\left.\sigma\right|_{\Lambda_{\text {even }}(V)}: \Lambda_{\text {even }}(V) \longrightarrow \Lambda_{\text {odd }}(V),
$$

and the universal construction takes $\sigma_{\mathcal{E}}$ to the operator

$$
\mathcal{E}_{M}=d+\delta: \Gamma\left(\Lambda_{\text {even }}\left(T^{*} M\right)\right) \longrightarrow \Gamma\left(\Lambda_{\text {odd }}\left(T^{*} M\right)\right),
$$

which is called the Euler operator on $M$. Its principal symbol will be denoted by $\sigma\left(\mathcal{E}_{M}\right)$. The Euler operator with coefficients in a vector bundle $E$ over $M$ will be denoted by $\mathcal{E}_{M} \otimes 1_{E} ;$ its principal symbol is $\sigma\left(\mathcal{E}_{M}\right) \otimes 1_{\pi^{*} E}$.

Now let $X \stackrel{i}{\hookrightarrow} M$ be an oriented Riemannian manifold of dimension $m$. The decomposition (9.13) induces the decompositions

$$
\begin{aligned}
\Lambda_{\text {even }}\left(\left.T M\right|_{X}\right) & =\left(\Lambda_{\text {even }}(T X) \otimes \Lambda_{\text {even }}(N X)\right) \oplus\left(\Lambda_{\text {odd }}(T X) \otimes \Lambda_{\text {odd }}(N X)\right), \\
\Lambda_{\text {odd }}\left(T M_{X}\right) & =\left(\Lambda_{\text {odd }}(T X) \otimes \Lambda_{\text {even }}(N X)\right) \oplus\left(\Lambda_{\text {even }}(T X) \otimes \Lambda_{\text {odd }}(N X)\right) .
\end{aligned}
$$

A straightforward computation shows that, in accordance with (9.22)-(9.23), the Euler operator can be represented on $X$ by the exterior tensor product

$$
\left.\mathcal{E}_{M}\right|_{X}=\left.\left(\mathcal{E}_{X} \# \mathcal{E}_{N X}\right)\right|_{X}
$$

of the Euler operator on $X$ by the family

$$
\mathcal{E}_{N X}=\left\{\mathcal{E}_{N_{x} X}\right\}_{x \in X}
$$

of Euler operators in the fibers of the normal bundle $N X$. Applying Theorem 8.10, we obtain the following assertion.

Theorem 9.5. The pullback on $X$ of the Euler operator on $M$ satisfies the following relation in the $K$-group $K\left(T^{*} X\right)$ :

$$
\left[i_{a}^{!}\left(\mathcal{E}_{M}\right)\right]=\left[\mathcal{E}_{X}\right]\left(\left[\Lambda_{\text {even }}(N X)\right]-\left[\Lambda_{\text {odd }}(N X)\right]\right) \in K\left(T^{*} X\right) .
$$

Remark 9.6. 1. By Theorem 8.9, the same relation holds for the metric trace of the Euler operator.

2. Let $E$ be an arbitrary vector bundle over $M$. Arguing as for the Hirzebruch operator, we obtain the following formula for the pullback of the Euler operator with coefficients in $E$ :

$$
\left[i_{a}^{!}\left(\mathcal{E}_{M} \otimes 1_{E}\right)\right]=\left[\mathcal{E}_{X}\right]\left(\left[\Lambda_{\text {even }}(N X) \otimes E\right]-\left[\Lambda_{\text {odd }}(N X) \otimes E\right]\right) \in K\left(T^{*} X\right) .
$$




\subsection{The Todd and Dirac operators}

Quite similar considerations give completely analogous results for the pullbacks of the Todd and Dirac operators. Hence, we give only statements of the corresponding theorems.

\subsubsection{The Todd (Cauchy-Riemann) operator}

Let $M$ be a complex-analytic manifold and $X \stackrel{i}{\hookrightarrow} M$ a complex-analytic submanifold. Then the normal bundle $N X$ is a complex vector bundle, and we write

$$
\Lambda_{\text {odd }}(N X)=\bigoplus_{j=1} \Lambda_{\bmod 2}^{j}(N X), \quad \Lambda_{\text {even }}(N X)=\bigoplus_{j=0} \Lambda_{\bmod 2} \Lambda^{j}(N X) .
$$

(Complexification is not needed.)

Theorem 9.7. Let $\mathcal{T}_{M}$ and $\mathcal{T}_{X}$ be the Todd operators (e.g., see [Pal65]) on $M$ and $X$, respectively, and let $E$ be an arbitrary complex vector bundle over $M$. Then the following relation holds in the group $K\left(T^{*} X\right)$ :

$$
\left[i_{a}^{!}\left(\mathcal{T}_{M} \otimes 1_{E}\right)\right]=\left[\mathcal{T}_{X}\right]\left(\left[\Lambda_{\text {even }}(N X) \otimes E\right]-\left[\Lambda_{\text {odd }}(N X) \otimes E\right]\right) \in K\left(T^{*} X\right) .
$$

\subsubsection{The Dirac operator}

Let $M$ be an oriented Riemannian manifold of dimension $2 N$ with a spinor structure, and let $X$ be an oriented Riemannian submanifold of dimension $2 n$, also equipped with a spinor structure, which is compatible with that on $M$.

Theorem 9.8. Let $\mathcal{D}_{M}$ and $\mathcal{D}_{X}$ be the Dirac operators on $M$ and $X$, respectively, and let $E$ be an arbitrary complex vector bundle over $M$. Then the following relation holds in the group $K\left(T^{*} X\right)$ :

$$
\left[i_{a}^{!}\left(\not_{M} \otimes 1_{E}\right)\right]=\left[\not D_{X}\right]\left(\left[\Delta_{+}(N X) \otimes E\right]-\left[\Delta_{-}(N X) \otimes E\right]\right) \in K\left(T^{*} X\right),
$$

where the $\Delta_{ \pm}(V)$ are the half-spin representations of the group $\operatorname{Spin}(2(N-n))$ corresponding to a $\operatorname{Spin}(2(N-n))$-module $V$.

\section{Appendix. Fourier Integral Operators}

Here we recall some well-known material from the theory of Fourier integral operators and simultaneously introduce the notation used in the main body of the article. For details, 
the reader should refer himself to Hörmander's book [Hör85] and also to [MSS90]. Our notation is close to that used in the latter book. We do not give any precise statements of theorems from these sources, just because the Fourier integral operators emerging in the theory of Sobolev problems are a special case not covered by the general theorems. Accordingly, the material given here serves only as a motivation for our reasoning. Precise statements of theorems for our special case are given at appropriate places in the main text.

\section{A.1 Homogeneous Lagrangian manifolds}

Let $X$ and $Y$ be $C^{\infty}$ manifolds of dimensions $m=\operatorname{dim} X$ and $n=\operatorname{dim} Y$. The cotangent bundles $T^{*} X$ and $T^{*} Y$ bear the canonical 2-forms (symplectic forms) $\omega_{X}^{2}$ and $\omega_{Y}^{2}$, whose expressions in canonical local coordinates $(x, p)$ and $(y, q)$ on $T^{*} X$ and $T^{*} Y$, respectively, are

$$
\omega_{X}^{2}=\sum_{j=1}^{m} d p_{j} \wedge d x_{j} \equiv d p \wedge d x, \quad \omega_{Y}^{2}=\sum_{j=1}^{n} d q_{j} \wedge d y_{j} \equiv d q \wedge d y .
$$

The group $\mathbb{R}_{+}$of positive numbers naturally acts on $T^{*} X$ (and $T^{*} Y$ ) by multiplication in the fibers. Let us equip the product $T^{*} X \times T^{*} Y=T^{*}(X \times Y)$ with the canonical form equal to the difference of the canonical forms on the factors:

$$
\omega_{X Y}^{2} \stackrel{\text { def }}{=} \omega_{X}^{2}-\omega_{Y}^{2}
$$

A submanifold

$$
L \stackrel{i}{\hookrightarrow} T^{*}(X \times Y)
$$

is called a homogeneous Lagrangian manifold if the following conditions hold:

Lagrangian property: $i^{*} \omega_{X Y}^{2} \equiv 0$;

maximum dimension: $\operatorname{dim} L=m+n$;

homogeneity: $L$ is $\mathbb{R}_{+}$-invariant, and

$$
L \subset T_{0}^{*}(X \times Y) \equiv T^{*}(X \times Y) \backslash\{0\},
$$

where $\{0\}$ is the zero section of $T^{*}(X \times Y)$.

The following additional condition is often imposed on a homogeneous Lagrangian manifold $L$.

Condition A.1. The manifold $L$ is closed in $T_{0}^{*}(X \times Y)$ and is contained in $T_{0}^{*} X \times T_{0}^{*} Y$.

In particular, it is used in general boundedness and composition theorems for Fourier integral operators (see below). However, the Lagrangian manifolds corresponding to most of the operators considered in this paper (including boundary, coboundary, and Green operators) do not satisfy this condition. That is why they need special treatment and display a variety of new effects. 


\section{A.2 Local description of homogeneous Lagrangian manifolds}

Let $L$ be a homogeneous Lagrangian manifold in $T^{*}(X \times Y)$. By the lemma on local coordinates [Arn89], the manifold $L$ can be covered by $\mathbb{R}_{+}$-invariant coordinate charts such that the coordinates in each of the charts are of the form $\left(x_{I}, p_{\bar{I}}, y_{J}, q_{\bar{J}}\right)$, where $(x, p)$ and $(y, q)$ are canonical local coordinates on $T^{*} X$ and $T^{*} Y$, respectively, $I \subset\{1, \ldots, m\}$, $J \subset\{1, \ldots, n\}, \bar{I}=\{1, \ldots, m\} \backslash I, \bar{J}=\{1, \ldots, m\} \backslash J, x_{I}=\left\{x_{j}\right\}_{j \in I}$, etc. These coordinates are referred to as canonical coordinates, and the corresponding canonical chart on $L$ will be denoted by $U_{I J}$. Thus, we have the coordinate map

$$
\psi_{I J} \equiv\left(x_{I}, p_{\bar{I}}, y_{J}, q_{\bar{J}}\right): U_{I J} \longrightarrow V_{I J} \subset \mathbb{R}^{m+n}
$$

In the chart $U_{I J}$, the manifold $L$ is specified by a uniquely determined generating function (action) $S_{I J}\left(x_{I}, p_{\bar{I}}, y_{J}, q_{\bar{J}}\right)$ defined in $V_{I J}$ and first-order homogeneous ${ }^{8}$ in $\left(p_{\bar{I}}, q_{\bar{J}}\right)$ such that the equations of $L$ read

$$
p_{I}=\frac{\partial S_{I J}}{\partial x_{I}}, \quad x_{\bar{I}}=-\frac{\partial S_{I J}}{\partial p_{\bar{I}}}, \quad q_{J}=-\frac{\partial S_{I J}}{\partial y_{J}}, \quad y_{\bar{J}}=\frac{\partial S_{I J}}{\partial q_{\bar{J}}} .
$$

(The signs in these equations are due to the fact that $\omega_{X}^{2}$ and $\omega_{Y}^{2}$ occur with opposite signs in $\omega_{X Y}^{2}$.)

\section{A.3 Composition of homogeneous Lagrangian manifolds}

Let $L_{1} \in T^{*} M_{1} \times T^{*} M_{2}$ and $L_{2} \in T^{*} M_{2} \times T^{*} M_{3}$ be homogeneous Lagrangian manifolds. We can view these manifolds as relations in $T^{*} M_{1} \times T^{*} M_{2}$ and $T^{*} M_{2} \times T^{*} M_{3}$, respectively (that is why they are called "homogeneous canonical relations" in Hörmander's writings), and then we can define the composition $L_{1} \circ L_{2}$ as the product of these relations:

$$
L_{1} \circ L_{2} \stackrel{\text { def }}{=}\left\{(u, v) \in T^{*} M_{1} \times T^{*} M_{3} \mid \exists w \in T^{*} M_{2}:(u, w) \in L_{1},(w, v) \in L_{2}\right\} .
$$

If the composition (A.2) is clean (i.e., $L_{1} \times L_{2}$ intersects $T^{*} M_{1} \times \Delta_{T^{*} M_{2}} \times T^{*} M_{3}$, where $\Delta_{T^{*} M_{2}}$ is the diagonal in $T^{*} M_{2} \times T^{*} M_{2}$, in a manifold $\widetilde{L}$ with tangent plane everywhere equal to the intersection of tangent planes of intersecting manifolds), proper (i.e., the projection $\widetilde{L} \longrightarrow T^{*}\left(M_{1} \times M_{3}\right)$ is proper) and connected (i.e., the set $L_{(u, v)}$ of points of $\widetilde{L}$ projected to the same point $(u, v) \in L_{1} \times L_{2}$ is connected), then the composition $L=L_{1} \circ L_{2}$ is also a homogeneous Lagrangian manifold. The dimension $e=\operatorname{dim} L_{(u, v)}$ of the fiber of the projection $\widetilde{L} \longrightarrow L$ is called the excess of the clean intersection.

\footnotetext{
${ }^{8}$ Since $L$ is homogeneous, it follows that $\bar{I}$ or $\bar{J}$ is not empty.
} 


\section{A.4 Definition of Fourier integral operators}

Let $L$ be a homogeneous Lagrangian manifold in $T^{*}(X \times Y)$. Suppose that $L$ is equipped with a quantized measure $\mu$ (e.g., see [MSS90] and references therein). Then Maslov's canonical operator $\mathcal{K} \equiv \mathcal{K}_{L, \mu}$ that takes asymptotically homogeneous functions on $L$ to distributions on $X \times Y$ is well defined (e.g., see [NOSS81] and appendix [MSS90]; for the original (small parameter) canonical operator, see [Mas72]).

A Fourier integral operator

$$
\widehat{\Phi}: C^{\infty}(Y) \longrightarrow \mathcal{D}^{\prime}(X)
$$

associated with $L$ in $T^{*}(X \times Y)$ can be defined as follows. One takes a smooth asymptotically homogeneous amplitude function $a$ on $L$ and takes $\widehat{\Phi}$ to be the operator with the (distributional) Schwartz kernel $\mathcal{K} a$. The local description can be obtained in the following manner. Let us cover $L$ with canonical charts. Consider some canonical chart $U_{I J}$. Let $a\left(x_{I}, p_{\bar{I}}, y_{J}, q_{\bar{J}}\right)$ be a smooth function supported in $V_{I J}$ and satisfying the estimates

$$
\begin{gathered}
\left|\frac{\partial^{\alpha+\beta+\gamma+\delta} a\left(x_{I}, p_{\bar{I}}, y_{J}, q_{\bar{J}}\right)}{\partial x_{I}^{\alpha} \partial p_{\bar{I}}^{\beta} \partial y_{J}^{\gamma} \partial q_{\bar{J}}^{\delta}}\right| \leq C_{\alpha \beta \gamma \delta}\left(1+\left|p_{\bar{I}}\right|+\left|q_{\bar{J}}\right|\right)^{r-|\beta|-|\delta|}, \\
|\alpha|+|\beta|+|\gamma|+|\delta|=0,1,2, \ldots
\end{gathered}
$$

for some $r \in R$. (In practice, one mostly deals with functions $a\left(x_{I}, p_{\bar{I}}, y_{J}, q_{\bar{J}}\right)$ asymptotically homogeneous in $\left(p_{\bar{I}}, q_{\bar{J}}\right)$.) The local Fourier integral operator with amplitude $a$ associated with the Lagrangian manifold $L$ in the chart $U_{I J}$ is defined as the integral operator $\widehat{\Phi}(L, a)$ with Schwartz kernel

$$
\begin{aligned}
K(x, y) & =\overline{\mathcal{F}}_{p_{\bar{I}} \rightarrow x_{\bar{I}}} \mathcal{F}_{q_{\bar{J}} \rightarrow y_{\bar{J}}}\left\{e^{i S_{I J}\left(x_{I}, p_{\bar{I}}, y_{J}, q_{\bar{J}}\right)} \widetilde{a}\left(x_{I}, p_{\bar{I}}, y_{J}, q_{\bar{J}}\right)\right\} \\
& \left.=\frac{i^{(m-n) / 2}}{(2 \pi)^{(m+n) / 2}} \iint e^{i\left[S_{I J}\left(x_{I}, p_{\bar{I}}, y_{J}, q_{\bar{J}}\right)+p_{\bar{T}} x_{\bar{T}}-q_{\bar{J}} y_{\bar{J}}\right.}\right] \widetilde{a}\left(x_{I}, p_{\bar{I}}, y_{J}, q_{\bar{J}}\right) d p_{\bar{I}} d q_{\bar{J}},
\end{aligned}
$$

where $\widetilde{a}=a \sqrt{\mu_{I}}$ and $\mu_{I}$ is the density of the measure $\mu$ in the coordinates of the chart $U_{I J}$. (Here $\mathcal{F}$ is the Fourier transform and $\overline{\mathcal{F}}$ the inverse Fourier transform. Products like $x_{I} p_{I}$ are defined as $x_{I} p_{I}=\sum_{j \in I} x_{j} p_{j}$.) Thus, $\widehat{\Phi}(L, a)$ acts as follows:

$$
[\widehat{\Phi}(L, a) \psi](x)=\int_{Y} K(x, y) \psi(y) d y .
$$

Global Fourier integral operators can be defined as sums of local Fourier integral operators in the charts, with usual precautions taken ensuring that all sums be locally finite etc. (This is of course not needed if $L / \mathbb{R}_{+}$is compact and hence can be covered by finitely many canonical charts.) The stationary phase method permits one to compare Fourier integral operators associated with charts that have nonempty intersections, and for the 
case of asymptotically homogeneous amplitudes one can define the notion of principal symbol of a Fourier integral operator, which is globally well-defined as a function on $L$. If $a$ is asymptotically homogeneous, then the leading part of $a$ is called the principal symbol of the Fourier integral operator. However, we do not dwell on these details, since in the main text we only deal with local expressions like (A.4).

\section{A.5 Pseudodifferential operators as Fourier integral operators}

An important example of Fourier integral operators occurs if one takes the diagonal

$$
L_{X}=\left\{(x, p, x, p) \mid(x, p) \in T_{0}^{*} X\right\} \subset T^{*}(X \times X)
$$

as the homogeneous Lagrangian manifold. The canonical coordinates on $L_{X}$ can always be taken in the form $\left(x, p^{\prime}\right)$ (where primes are used on the variables pertaining to the second factor $T^{*} X$ in the product $T^{*} X \times T^{*} X$ ), the generating function is $S=p^{\prime} x$, and one has

$$
\left[\widehat{\Phi}\left(L_{X}, a\right) \psi\right](x)=\left(\frac{1}{2 \pi}\right)^{m} \iint e^{i p^{\prime}\left(x-x^{\prime}\right)} a\left(x, p^{\prime}\right) d p^{\prime} d x .
$$

We see that $\widehat{\Phi}\left(L_{X}, a\right)$ is just the pseudodifferential operator with symbol $a(x, p)$.

Fourier integral operators possess a variety of properties, the main of which are boundedness properties, composition formulas, and the passage to the $L^{2}$-adjoint operator. Let us recall these properties.

\section{A.6 Boundedness theorems}

Boundedness theorems [Hör85] claim that Fourier integral operators associated with a Lagrangian manifold

$$
L \stackrel{i}{\hookrightarrow} T^{*}(X \times Y)
$$

extend to bounded operators between Sobolev spaces $H^{s}(Y)$ and $H^{l}(X)$ provided that $L$ satisfies condition A.1; the lower and upper bounds on the order $s-l$ depend on the order of the amplitude as well as on the corank of the lift of the canonical form $\omega_{X}^{2}$ (or, which is the same, $\omega_{Y}^{2}$ ) to $L$.

\section{A.7 Composition theorems}

Composition theorems for Fourier integral operators proved in [Hör85] establish that under mild conditions the product of two Fourier integral operators is again a Fourier integral operator. Namely, let $L_{1} \in T^{*} M_{1} \times T^{*} M_{2}$ and $L_{2} \in T^{*} M_{2} \times T^{*} M_{3}$ be Lagrangian manifolds, and let $\widehat{\Phi}_{1,2}$ be Fourier integral operators associated with $L_{1,2}$, respectively. Next, suppose that the composition $L=L_{1} \circ L_{2}$ (see (A.2)) is clean and that both $L_{1}$ and 
$L_{2}$ satisfy condition A.1. Then the product $\widehat{\Phi}_{1} \widehat{\Phi}_{2}$ is a Fourier integral operator associated with the Lagrangian manifold $L$. Moreover, if $a_{i}$ is the principal symbol of $\widehat{\Phi}_{i}$ (defined on the Lagrangian manifold $\left.L_{i}\right), i=1,2$, then the principal symbol $a(u, v)$ of $\widehat{\Phi}_{1} \widehat{\Phi}_{2}$ at a point $(u, v) \in L$ is equal to the integral of the direct product $a_{1} \times a_{2}=a_{1}(u, w) a_{2}(w, v)$ over the fiber $L_{(u, v)}$ of the bundle $\widetilde{L} \longrightarrow L$ (see Subsection A.3). A special case of this theorem occurs if one the manifolds $L_{1}$ and $L_{2}$ is the diagonal (or the identity relation) $I$. Since $I \circ L=L$ (and likewise $L \circ I=L$ ) for any Lagrangian manifold for which the composition is well defined, we see that the product of a Fourier integral operator associated with a Lagrangian manifold $L$ by a pseudodifferential operator is a Fourier integral operator associated with the same manifold $L$. Moreover, the excess of such compositions is always zero, and so the principal symbol of the product is just the product of the principal symbols of the factors (taken at appropriate points).

\section{A.8 $\quad L^{2}$-adjoints of Fourier integral operators}

Let $\widehat{\Phi}$ be a Fourier integral operator associated with a homogeneous Lagrangian manifold

$$
L \subset T^{*}\left(M_{1} \times M_{2}\right)
$$

Then the $L^{2}$-adjoint $\widehat{\Phi}^{*}$ of the operator $\widehat{\Phi}$ is also a Fourier integral operator. It is associated with the Lagrangian manifold

$$
{ }^{T} L=\left\{(v, u) \mid u \in T^{*} M_{1}, \quad, v \in T^{*} M_{2}, \quad(u, v) \in L\right\} \subset T^{*}\left(M_{2} \times M_{1}\right)
$$

obtained from $L$ by transposing the factors.

\section{A.9 Fourier integral operators on sections of vector bundles}

All preceding considerations pertain to the case of Fourier integral operators acting in function spaces, but the generalization to the case of operators acting in spaces of sections of vector bundles is straightforward. The (principal) symbol of a Fourier integral operators acting between spaces of sections of vector bundles is a morphism between the lifts of these bundles to the Lagrangian manifold.

\section{References}

[Arn89] V. I. Arnold, Mathematical Methods of Classical Mechanics, second ed., Graduate Texts in Mathematics, vol. 60, Springer-Verlag, Berlin-Heidelberg-New York, 1989 [translated from the Russian].

[AS63] M. F. Atiyah and I. M. Singer, The index of elliptic operators on compact manifolds, Bull. Amer. Math. Soc. 69 (1963), 422-433. 
[DN55] A. Douglis and L. Nirenberg, Interior estimates for elliptic systems of partial differential equaions, Comm. Pure Appl. Math. 8 (1955), 503-538.

[Esk73] G. I. Eskin, Boundary Value Problems for Elliptic Pseudodifferential Equations, Nauka, Moscow, 1973 [Russian].

[Hör83a] L. Hörmander, The Analysis of Linear Partial Differential Operators. I, Springer-Verlag, Berlin-Heidelberg-New York-Tokyo, 1983.

[Hör83b] — The Analysis of Linear Partial Differential Operators. II, SpringerVerlag, Berlin-Heidelberg-New York-Tokyo, 1983.

[Hör85] _ The Analysis of Linear Partial Differential Operators. IV, SpringerVerlag, Berlin-Heidelberg-New York-Tokyo, 1985.

[Mas72] V. P. Maslov, Théorie des perturbations et méthod asymptotiques, Dunod, Paris, 1972 [French transl. from the Russian 1965 edition].

[MSS90] A. Mishchenko, V. Shatalov, and B. Sternin, Lagrangian manifolds and the Maslov operator, Springer-Verlag, Berlin-Heidelberg, 1990.

[Mon71] L. Boutet de Monvel, Boundary problems for pseudodifferential operators, Acta Math. 126 (1971), 11-51.

[NOSS81] V. E. Nazaikinskii, V. G. Oshmyan, B. Yu. Sternin, and V. E. Shatalov, Fourier integral operators and the canonical operator, Uspekhi Mat. Nauk 36 (1981), no. 2, 81-140.

[NS66a] S. P. Novikov and B. Yu. Sternin, Traces of elliptic operators on submanifolds and K-theory, Dokl. Akad. Nauk SSSR 1265-1268 170 (1966), no. 5, 12651268 .

[NS66b] _ Elliptic operators and submanifolds, Dokl. Akad. Nauk SSSR 525-528 171 (1966), no. 6, 525-528.

[Pal65] R. S. Palais, Seminar on the Atiyah-Singer Index Theorem, Princeton Univ. Press, Princeton, NJ, 1965.

[Rha55] G. de Rham, Variétès différentiables, Hermann, Paris, 1955.

[Sch91] B.-W. Schulze, Pseudodifferential Operators on Manifolds with Singularities, North-Holland, Amsterdam, 1991.

[Sob37] S. L. Sobolev, On a boundary value problem for the polyharmonic equation, Matem. Sbornik 2 (1937), no. 3, 467-500. 
[Stb64] S. Sternberg, Lectures on Differential Geometry, Prentice Hall, Englewood Cliffs, N.J., 1964.

[Ste64] B. Yu. Sternin, General boundary value problems for elliptic equations in a region whose boundary consists of manifolds of different dimension, Dokl. Akad. Nauk SSSR 159 (1964), no. 5, 992-994.

[Ste66] - Elliptic and parabolic problems on manifolds with boundary consisting of components of different dimension, Trudy Mosk. Mat. Obshch. 15 (1966), $38-108$.

[Ste67] _ Elliptic (co)boundary morphisms, Dokl. Akad. Nauk SSSR 172 (1967), no. $1,44-47$.

[Ste71] - Topological aspects of the Sobolev problem, Moscow Institute of Electronic Engineering, Moscow, 1971 [Russian].

[Ste76] _ Relative elliptic theory and the Sobolev problem, Dokl. Akad. Nauk SSSR 230 (1976), no. 2, 287-290.

[SS92a] B. Yu. Sternin and V. E. Shatalov, Fourier-Maslov integral operators associated with a bundle and an extension of the algebra of pseudodifferential operators, Dokl. Ross. Akad. Nauk 323 (1992), no. 1, 25-29.

[SS92b] — A class of nonlocal elliptic problems, Dokl. Ross. Akad. Nauk 323 (1992), no. 2, 245-249.

[SS94] - Extension of the algebra of pseudodifferential operators and some nonlocal elliptic problems, Mat. Sbornik 185 (1994), no. 3, 117-159.

[SS96] _ Relative elliptic theory and the Sobolev problem, Mat. Sbornik 187 (1996), no. 11, 115-144.

[VE65a] M. I. Vishik and G. I. Eskin, Convolution equations in a bounded region, Uspekhi Mat. Nauk 20 (1965), no. 3, 89-152.

[VE65b] _ Elliptic convolution equations in a bounded region and their applications, Uspekhi Mat. Nauk 22 (1965), no. 1, 15-76. 